\title{
Learning State-Based Behavior Using Deep Neural Networks
}

\author{
by
}

\section{Mohamed Zalat}

A thesis submitted to the Faculty of Graduate and Postdoctoral Affairs in partial fulfillment of the requirements for the degree of

\section{Master of Applied Science}

in

Electrical and Computer Engineering

\author{
Carleton University \\ Ottawa, Ontario
}

(C) 2021

Mohamed Zalat 


\section{Abstract}

Imitation learning is a supervised learning problem that involves training a model to perform a task in a given environment using demonstrations of an expert. In this thesis, we propose 5 metrics to evaluate the performance of imitation learning agents. We compare state-of-the-art imitation learning models to deep neural networks at imitating state-based and reactive behavior. To compare the imitation learning techniques, we use two partially observable domains: the continuous RoboCup domain and the discrete Vacuum Cleaner domain. We show how our proposed metrics provide us with more qualitative information about the performance of imitation learners when imitating state-based behavior compared to state-of-the-art metrics. In addition, we show how our testing methodology provides results that resemble the eye-test that current testing methodologies fail to provide. We also show how Long Short-Term Memory (LSTM) networks outperform state-of-the-art models at imitating state-based behavior in the RoboCup soccer domain. 


\section{Acknowledgements}

Firstly, I would like to give special thanks to my supervisor, Professor Babak Esfandiari, who provided me with valuable guidance and feedback throughout my dissertation. Without your feedback and guidance, this work would have not been possible.

I would also like to give special thanks to my co-supervisor, Professor Ana-Maria Cretu, who supported me during my dissertation and provided me with valuable feedback on my final revisions.

I dedicate this work to Mom, Dad, Youssef, Nour, and May who supported me throughout my dissertation. I would not be able to do this without you.

I would also like to thank my friends that were there for me through the ups and downs of my dissertation: Zaidoon and Adnan. 


\section{Contents}

Abstract $\quad$ i

$\begin{array}{ll}\text { Acknowledgements } & \text { ii }\end{array}$

List of Tables $\quad$ viii

List of Figures $\quad$ xi

Acronyms $\quad$ xiii

1 Introduction 1

1.1 Motivation . . . . . . . . . . . . . . . . . . . . 1

1.1.1 Imitation Learning . . . . . . . . . . . . . . . . . . . 1

1.1.2 State-Based Behavior . . . . . . . . . . . . . . 2

1.1.3 Long Short-Term Memory . . . . . . . . . . . . . . . 3

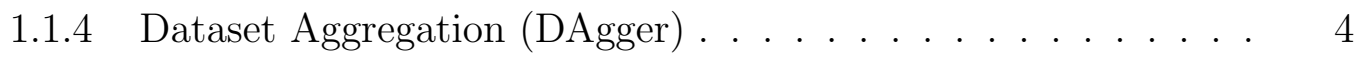

1.1.5 Testing Methodologies . . . . . . . . . . . . 5

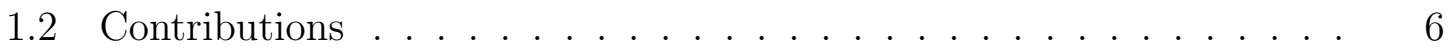

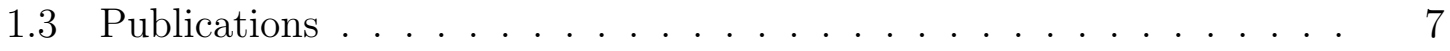

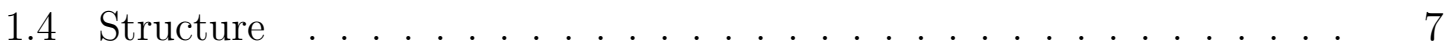


2 Background $\quad 9$

2.1 Introduction . . . . . . . . . . . . . . . . . . 9

2.2 Agents: General Definitions . . . . . . . . . . . . . . . . 10

2.3 Imitating Reactive Experts . . . . . . . . . . . . . . . . . . 12

2.4 Imitating State-based Experts . . . . . . . . . . . . . . . . . 13

2.4.1 LSTM .............................. 14

2.5 Dealing With the Dependency of Environment States on Actions . . . 19

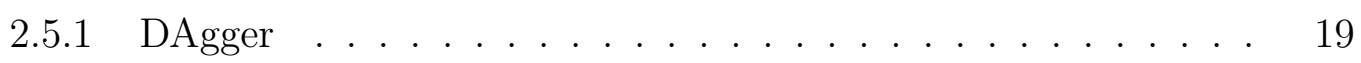

2.6 Conclusion . . . . . . . . . . . . . . . . . . . 21

3 Related Work $\quad 22$

3.1 Introduction . . . . . . . . . . . . . . . . . . 22

3.2 Benchmarks . . . . . . . . . . . . . . . . . 23

3.2.1 Introduction . . . . . . . . . . . . . . . 23

3.2.2 Vacuum Cleaner Domain . . . . . . . . . . . . . 23

3.2.2.1 Introduction ................. 23

3.2.2.2 Vacuum Cleaner With Dirt . . . . . . . . . . . 24

3.2.2.3 Vacuum Cleaner Without Dirt . . . . . . . . 28

3.2.2.4 Conclusion . . . . . . . . . . . . . . . . 30

3.2.3 RoboCup Soccer Domain . . . . . . . . . . . . . . . . 30

3.2.3.1 Introduction . . . . . . . . . . . . 30

3.2.3.2 Discretized RoboCup Soccer Model . . . . . . . . . . 31

3.2.3.3 Conclusion .................... 32

3.2.4 Conclusion . . . . . . . . . . . . . . . . . . . . . 33

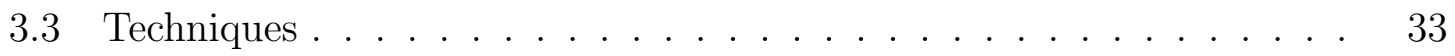

3.3.1 Introduction . . . . . . . . . . . . . . . . 33 
3.3.2 Temporal Backtracking . . . . . . . . . . . . . . . . . . 34

3.3.3 Dynamic Bayesian Networks . . . . . . . . . . . . . . . . 37

3.3.4 Probabilistic Finite Automata . . . . . . . . . . . . . . . 40

3.3.5 Case-Based Reasoning . . . . . . . . . . . . . . . . . . 42

3.3 .6 Conclusion . . . . . . . . . . . . . . . . . . . 46

3.4 Testing Methodologies and Metrics _ . . . . . . . . . . 47

3.4 .1 Introduction . . . . . . . . . . . . . . 47

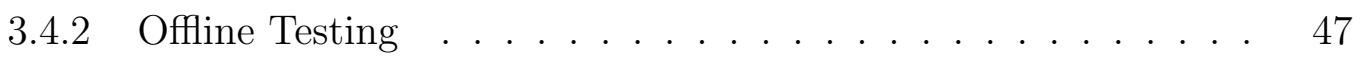

3.4 .3 Online Testing . . . . . . . . . . . . . . . . . . 49

3.4 .4 Conclusion . . . . . . . . . . . . . . . . . . . 50

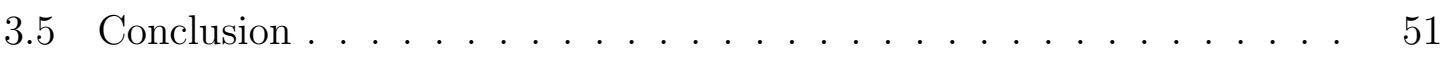

4 Methodology $\quad 53$

4.1 Introduction . . . . . . . . . . . . . . . . . . 53

4.2 Benchmarks . . . . . . . . . . . . . . . . . . . 54

4.2.1 Representing the Continuous Input from the RoboCup Soccer

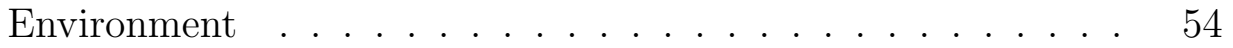

4.2.1.1 RoboCup Soccer Environment Space . . . . . . . . 55

4.2.1.2 Action Space . . . . . . . . . . . . . . 56

4.2 .1 .3 Conclusion . . . . . . . . . . . . . 57

$4.2 .2 \quad$ RoboCup Soccer Experts . . . . . . . . . . . . . . . . 58

4.2 .2 .1 Introduction . . . . . . . . . . . 58

4.2 .2 .2 Conclusion . . . . . . . . . . . . . . 62

4.2 .3 Conclusion . . . . . . . . . . . . . . . . . . . . . . 62

4.3 Techniques. . . . . . . . . . . . . . . . . . . . . 63

4.3 .1 Proposed Techniques . . . . . . . . . . . . . . . . . . . 64 
4.3.1.1 MLP . . . . . . . . . . . . . . 65

$4.3 .1 .2 \quad \operatorname{LSTM} \ldots \ldots \ldots \ldots \ldots$

4.4 Metrics and Testing Methodology . . . . . . . . . . 67

4.4 .1 Proposed Metrics . . . . . . . . . . . . . . . . 68

4.5 Conclusion . . . . . . . . . . . . . . . . . . . 73

$\begin{array}{lll}5 & \text { Experiments } & 75\end{array}$

5.1 Introduction . . . . . . . . . . . . . 75

5.2 Preliminary Experiment: Case-Based Reasoning (CBR) Similarity Metrics in RoboCup Soccer . . . . . . . . . . . . . . . . . . 77

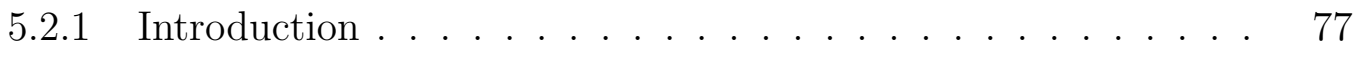

5.2 .2 Results and Discussion . . . . . . . . . . . . . . . 79

5.2 .3 Conclusion . . . . . . . . . . . . . . . . . . 82

5.3 The Effect of DAgger on Imitation Learning Techniques . . . . . . . 82

$5.3 .1 \quad$ DAgger Experiment Setup . . . . . . . . . . . . . . 84

5.3 .2 Results and Discussion . . . . . . . . . . . . . . 85

5.3 .3 Conclusion . . . . . . . . . . . . . . . . . . . . . 91

5.4 RoboCup Soccer Experiments . . . . . . . . . . . . . . . . . 92

5.4 .1 Introduction . . . . . . . . . . . . . . 92

5.4 .2 RoboCup Soccer Experiment Setup . . . . . . . . . . . . 93

$5.4 .3 \quad$ Krislet Expert Results . . . . . . . . . . . . . . . . . . 95

5.4.4 Switch Expert Results . . . . . . . . . . . . . . . 96

5.4.5 Offense Expert Results . . . . . . . . . . . . . . . . . 97

$5.4 .6 \quad$ Pro Expert Results . . . . . . . . . . . . . . . . . . . . . 99

5.4 .7 Goal Expert Results . . . . . . . . . . . . . . . . . . 101

5.4 .8 Pro Goal Expert Results . . . . . . . . . . . . . . . . . . 102 
5.4 .9 Conclusion . . . . . . . . . . . . . . . . . . . . . . . 103

5.5 Vacuum Cleaner Experiments . . . . . . . . . . . . . . . . 104

5.5 .1 Introduction . . . . . . . . . . . . . . . . . . . . 104

5.5.2 An Experiment of Testing Methodologies . . . . . . . . . . 105

5.5.2.1 Experiment Setup . . . . . . . . . . . . . 106

5.5 .2 .2 Results . . . . . . . . . . . . . . . . . . 108

5.5.2.3 Conclusion . . . . . . . . . . . . . . . . 112

5.5.3 Imitation Learning Performance of LSTM and Multi-Layer Perceptron $(\mathrm{MLP}) \ldots \ldots \ldots \ldots$

5.5.3.1 Introduction . . . . . . . . . . . . . . . 113

5.5.3.2 Experiment Setup . . . . . . . . . . . . . . . 113

5.5 .3 .3 Results . . . . . . . . . . . . . . . 114

5.5.3.4 Conclusion . . . . . . . . . . . . . . . 121

5.6 Conclusion . . . . . . . . . . . . . . . . . . . . . . . . . . . . . . . . . . 122

6 Conclusion and Future Work 124

6.1 Conclusion . . . . . . . . . . . . . . . . . . . . . . . . . . . . 124

6.2 Future Work . . . . . . . . . . . . . . . . . . . . . 127

$\begin{array}{ll}\text { Bibliography } & 129\end{array}$ 


\section{List of Tables}

3.1 The experts used in the model of the vacuum cleaner domain that includes dirt. Those experts were used in the work of Ontañón et al. [1] and some of them were used in the work Gunaratne et al. [2] . . . 26

3.2 The expert used in the study of Gunaratne et al. [2] This expert was used in the vacuum cleaner with dirt model. . . . . . . . . . . . . 27

3.3 The experts used in the work of Tîrnăucă et al. [3] with the vacuum cleaner model that excludes dirt. . . . . . . . . . . . . . . . . . 29

3.4 The experts used in the study of Gunaratne et al. [4] using the discretized model of RoboCup soccer. . . . . . . . . . . . . . . . . . . . . 32

4.1 Description of the environment vector features . . . . . . . . 55

4.2 The action space of the agent $\ldots \ldots \ldots \ldots \ldots$

4.3 Example environment state frequencies of the Krislet expert and the MLP agent in the RoboCup soccer domain. . . . . . . . . . . 72

5.1 The results of the n-ordered similarity and weighted similarity CBR agents at imitating the Switch expert. . . . . . . . . . .

5.2 The results of the n-ordered similarity and weighted similarity CBR agents at imitating the Offense expert. . . . . . . . . . . . . 81 
5.3 The results of the n-ordered similarity at imitating the experts before and after the application of DAgger. . . . . . . . . . . . 86

5.4 The results of Temporal Backtracking (TB) at imitating the experts before and after the application of DAgger. . . . . . . . . . . . 87

5.5 The performance metrics of MLP at imitating the experts before and after the application of DAgger. . . . . . . . . . . . . . . . . . . 89

5.6 The performance metrics of LSTM at imitating the experts before and after the application of DAgger. . . . . . . . . . . . . . . . 90

5.7 The final training set size used by the MLP agent to imitate each expert. 94

5.8 The final training set size used by the LSTM agent to imitate each expert. 94

5.9 The results of imitating the Krislet expert. . . . . . . . . . . . . . . 95

5.10 The results of imitating the Switch expert. . . . . . . . . . . . . . 97

5.11 The results of imitating the Offense expert. . . . . . . . . . . . 98

5.12 The results of imitating the Pro expert. . . . . . . . . . . . . . 99

5.13 The results of imitating the Goal expert. . . . . . . . . . . . . . 101

5.14 The results of imitating the Pro Goal expert. . . . . . . . . . . . . . 102

5.15 A comparison between the F1 scores obtained using the methodology of Gunaratne et al. [4] (the offline macro F1) and the results obtained using our methodology when imitating the Smart Random expert. . . 108

5.16 A comparison between the F1 scores obtained using the methodology of Gunaratne et al. [4] (the offline macro F1) and the results obtained using our methodology when imitating the Smart Straight Line expert. 109

5.17 A comparison between the F1 scores obtained using the methodology of Gunaratne et al. [4] (the offline macro F1) and the results obtained using our methodology when imitating the ZigZag expert. . . . . . . 110 
5.18 A comparison between the F1 scores obtained using the methodology of Gunaratne et al. [4] (the offline macro F1) and the results obtained using our methodology when imitating the Fixed Sequence expert. . . 111

5.19 The results of imitating the Smart Random expert. . . . . . . . . . . 114

5.20 The results of imitating the Smart Straight Line expert. . . . . . . . . 116

5.21 The results of imitating the ZigZag expert. . . . . . . . . . . . . 118

5.22 The results of imitating the Fixed Sequence expert. . . . . . . . . . 120 


\section{List of Figures}

2.1 An example Recurrent Neural Network (RNN) chain. Here, we can see how each internal state is updated based on the current environment state $s_{t} \in S$ and the previous internal state $i_{t-1} \in I$. An action $a_{t} \in A$ is taken at each time step $t$. The cells highlighted in orange represent an RNN cell unrolled through time. . . . . . . . . . . . . 15

2.2 The vanilla RNN cell. Here, we indicate that the tanh function contains parameters that are learnt by enclosing it in a red box. $i_{t}$ and $a_{t}$ are computed using the functions in Equation (2.10) and Equation (2.11).

2.3 The LSTM cell. Here, the functions that contain parameters that are learnt by enclosed in a blue box. Functions that do not contain learnt parameters are contained in a grey box/circle. The red arrows here represent the "gradient freeway" of the LSTM cell that addresses the gradient vanishing/exploding problem of vanilla RNN. . . . . . . .

3.1 An example Bayesian Network where the action $A$ is dependent upon the environment state $S$. Note that $S$ and $A$ can be multi-dimensional. 38 
3.2 Learning from Observation Dynamic Bayesian Network (LfODBN) Model. $S_{t}$ is the environment state, $A_{t}$ is the action, and $I_{t}$ is the hidden internal state of the agent. . . . . . . . . . . . .

3.3 Input-Output Hidden Markov Model. Note how the hidden internal state node does not depend on the previous action. . . . . . . . .

4.1 Krislet Expert Decision Tree . . . . . . . . . . . . . . . . . . . 59

4.2 Switch Expert Automaton . . . . . . . . . . . . . . 60

4.3 Offense Expert Automaton . . . . . . . . . . . . . . . . 60

4.4 Pro Expert Automaton . . . . . . . . . . . . . . . . . 60

4.5 Goal Expert Automaton . . . . . . . . . . . . . . . . 61

4.6 The online testing methodology of obtaining the F1 score. The blue action is the action performed in the environment, and the expected action is what is used to calculate the F1 score. . . . . . . . . . . 67 


\section{Acronyms}

Adam . . . . . . . . . adaptive learning rate optimization

BN $\ldots \ldots \ldots \ldots \ldots$ Bayesian Network

CBR ............ Case-Based Reasoning

DAgger ............. Dataset Aggregation

DBN . . . . . . Dynamic Bayesian Network

EM . . . . . E . Expectation-Maximization

GRU . . . . . . . . Gated Recurrent Unit

IOHMM $\ldots \ldots \ldots \ldots \ldots$ Input-Output Hidden Markov Model

$\mathrm{kNN} \ldots \ldots \ldots \ldots \ldots \ldots$ k-Nearest Neighbors

LfODBN . . . . . . . . Learning from Observation Dynamic Bayesian Network

LSTM . . . . . . . Long Short-Term Memory 


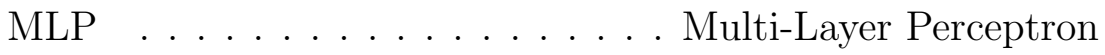

NLP . . . . . . . . . . . Natural Language Processing

$\mathrm{PFA} \ldots \ldots \ldots$. . . . . Probabilistic Finite Automata

RNN ............. Recurrent Neural Network

TB ................. Temporal Backtracking 


\section{Chapter 1}

\section{Introduction}

\subsection{Motivation}

\subsubsection{Imitation Learning}

Imitation learning is a problem that involves learning the behavior of an expert using demonstrations of the expert performing a task in an environment, where the expert can be a human or a computer program [5]. The goal of imitation learning is to generalize the behavior of the expert so that the learner can imitate the expert in future unseen situations. Demonstrations of the expert are sequences of environment state-action pairs. Typically, the input of a trained imitation learning agent is the current environment state and its output is an action. However, its input can be a sequence of environment state-action pairs depending on the imitation learning technique and the type of behavior it intends to imitate. For example, state-based Case-Based Reasoning (CBR) approaches use the trajectory of the agent to select which action to take instead of the current environment state. Imitation learning has been successfully used to train agents that imitate experts in many domains, such as 
the RoboCup soccer domain [6], and the vacuum cleaner domain [3]. Moreover, it has been used in robotic applications, such as picking up and placing objects [7, 8], and obstacle avoidance [9].

There are some advantages to the use of imitation learning versus the use of reinforcement learning. Humans that may not be experts in robotics interact with robots for different applications such as home use [10]; therefore, we can expect them to have an idea of what a robot should be able to do. Thus, a non-robotics-expert should ideally be able to adapt the robot to perform a task they wish to perform. Reinforcement learning requires designing a reward function which is known to be difficult and requires considerable expertise [11]. On the other hand, humans with no expertise in robotics can teach a robot how to perform a task through imitation learning [12]. For example, we can teach a robot to handle objects by remotely operating the robot and providing it with demonstrations of grabbing and handling objects instead of designing a reward function $[7,8]$.

\subsubsection{State-Based Behavior}

A reactive agent is an agent that does not use information from the past and only relies on the current environment state to select an action [13]. For example, a thermometer is a reactive agent that provides a reading based on the current temperature of the environment. Hence, a reactive agent does not need any form of memory or features that provide information on the past.

Most domains have experts that include information from past environment states or actions when deciding which action to take. For example, a competent soccer player decides which way to turn when they lose sight of the ball based on the last direction of the ball. Such experts are said to exhibit state-based behavior [13]. Information 
from past environment states or actions can be modelled as a hidden internal state of the expert that is invisible to the imitation learner.

Assuming that the imitation learner has access to the same information as the expert, learning reactive behavior is much easier than learning state-based behavior because the expert does not use information invisible to the imitation learner. In contrast, proper imitation of state-based behavior requires indirectly learning the hidden internal states of the expert. In this thesis, we compare the performance of different state-based imitation learning techniques at imitating state-based behavior including Long Short-Term Memory (LSTM) networks, assuming that the imitation learner has access to the same information as the expert.

\subsubsection{Long Short-Term Memory}

While many features that a state-based expert considers from its past can be included in the input of the imitation learner, some of them are not obvious. For example, the last direction a soccer player saw the ball in is a feature that can be easily included in the input of an imitation learner to imitate a competent soccer player. However, the soccer player may be taking actions using past information that is not as trivial and easy to encode in the input of the imitation learner. Therefore, it is impractical to pre-include all features that a black-box expert considers from past observations in the set of input features of an imitation learning agent. This motivates us to use models that innately consider past environment states when deciding which action to take.

A Long Short-Term Memory (LSTM) network is a Recurrent Neural Network (RNN) [14]. A RNN attempts to learn two functions: a function that maps its current input and previous hidden state to the current hidden state, and a function that maps the current hidden state and current input to the output of the network [15]. This 
hidden state is analogous to the hidden internal state of a state-based expert. Thus, a LSTM network should be capable of learning state-based behavior in theory.

LSTMs have been used in many domains that require handling sequential data such as Natural Language Processing (NLP) applications [16, 17], and various biomedical applications such as predicting protein-protein interaction $[18,19]$. However, no stateof-the-art research in imitation learning and behavioral cloning in the RoboCup soccer and vacuum cleaner domains make use of LSTM networks to imitate state-based experts $[1,2,3,4,20]$. Instead, it is common to use Case-Based Reasoning (CBR) techniques $[2,20]$ and probabilistic graphical models $[1,4]$. The performance of those techniques at imitation learning was also never compared to RNNs such as LSTM networks.

\subsubsection{Dataset Aggregation (DAgger)}

While imitation learning simplifies the learning problem to a supervised learning problem, it still has its own challenges. One difficulty of imitation learning is that the actions of the agent influence its environment state. Hence, it does not comply with the i.i.d. assumption of most machine learning models [21]. This results in issues that are prevalent during both the training and testing of imitation learning agents. For example, small deviations from the expert's behavior during execution of an imitation learning agent cascade into larger deviations because the environment state is influenced by the agent's behavior.

Dataset Aggregation (DAgger) is an iterative algorithm that attempts to deal with this problem during training [21]. It involves using the trained agent to generate a trajectory, then using the expert to provide feedback on which action the agent should have performed at every step. The trajectory with the corrected actions is then added 
to the training set of the agent and the agent is retrained. This process is repeated till the trained agent's behavior converges to the behavior of the expert. This iterative process aims to minimize the disparity between the environment state distribution of the training set and the trained agent.

We make use of DAgger in many of our experiments to deal with this issue. We will also investigate the effect of DAgger on different imitation learning techniques later in this thesis.

\subsubsection{Testing Methodologies}

Currently, testing methodologies that compare the performance of different techniques at imitating state-based experts mostly employ offline testing and make use of the predictive accuracy and F1 score. Offline testing is a testing methodology where the results of the trained agent are obtained by asking it to predict the actions it should take along a pre-existing trajectory of the expert. We refer to it as offline testing because it does not involve running the trained agent in its environment to allow the trained agent to produce its own trajectory.

When a trained agent is deployed in the environment, its actions affect its own trajectory and hence, affect its future actions. Thus, the offline testing methodology produces results that are not always consistent with the qualitative performance of the agents when they are deployed. Therefore, we need to test the agents by deploying them in the environment wherever possible to obtain reliable results. We will refer to this method of testing as online testing.

Other work in the field of imitation learning involves the use of hand crafted metrics for the domain of the agent $[21,22]$. While this provides accurate results, those metrics cannot be used in other domains. Moreover, when this metric is simply 
a score of the imitation learning agent in its application domain as in [21], we are making the assumption that the expert's goal is to maximize or minimize that specific metric. Such metrics are evaluating how well the agent is performing in the domain, as opposed to how well the agent is imitating the expert. A simple example of a domain specific metric that would fail to capture how well an agent is imitating an expert is the number of goals an agent scores in a soccer simulation. In this example, an agent may not be imitating the expert's behavior and score more goals than the expert, making the metric inadequate in capturing how well the agent imitates the expert's behavior. In this thesis, we will propose generic metrics that measure how well the agent generalizes the behavior of the expert. We hypothesize that those metrics will provide information on how well an agent imitates the expert's behavior.

\subsection{Contributions}

In this thesis, we made multiple contributions to the field of state-based imitation learning. We list the contributions below:

- We propose a LSTM network to imitate state-based behavior.

- We propose the use of five new metrics that involve measuring the distance between the trajectory of a trained agent and the expert. We will show how those metrics provide more reliable results to evaluate state-based imitation learning techniques than the F1 score.

- We compare the online testing methodology, that involves running the trained agent in its environment, to the offline testing methodology, which entails using the trained agent to predict actions on an existing trajectory of the expert. 
We will show how the offline testing methodology produces unreliable results; whereas, the online testing methodology produces results consistent with the performance of the agents.

- We investigate the effect of DAgger on state-based imitation learning techniques. We will show how it can negatively affect the performance of some state-based imitation learning techniques.

\subsection{Publications}

One peer reviewed publication resulted from the work of this thesis:

- Mohamed Zalat and Babak Esfandiari. Performance metrics for state-based imitation learning. To appear in The International FLAIRS Conference Proceedings, volume 34, 2021

\subsection{Structure}

In Chapter 2, we provide the background required to understand the contributions of this thesis. Chapter 3 contains the state-of-the-art state-based imitation learning techniques, benchmarks, and testing methodologies. Chapter 4 presents our proposed model and experts of the RoboCup soccer benchmark, the architecture and training methodology of our proposed LSTM and Multi-Layer Perceptron (MLP) models, and our testing methodology. Moreover, it contains the five performance metrics we propose for state-based imitation learning. Chapter 5 contains the setup and results

of our experiments, including the hyper-parameters and datasets we used for each 
imitation learning technique. Chapter 6 contains the conclusion of this thesis and future work. 


\section{Chapter 2}

\section{Background}

\section{$2.1 \quad$ Introduction}

In this chapter, we aim to introduce the terminologies and definitions required to understand the rest of this thesis. Moreover, we aim to provide the background knowledge and the reasons behind some of the techniques that we will use in later chapters of this thesis. In the first section of this chapter, we will provide the formal definition of an agent and present two types of agents: reactive agents and state-based agents. In the section that follows, we will provide some background on imitation learning in the context of imitating reactive agents. Next, we will present the problems that imitation learning introduces in the context of imitating state-based agents and how we can address them. Lastly, we will present a technique used to deal with the inherent problem of imitation learning: the dependency of the environment state of the agent on the previous action of the agent. 


\subsection{Agents: General Definitions}

Wooldridge [13] defines an agent as "a computer system that is situated in some environment, and that is capable of autonomous action in this environment in order to meet its design objectives." We will notate the set of possible environment states of an agent with $S$, and the set of actions that the agent can take with $A$.

Using the definition of Wooldridge [13], we can see that the trajectory/run of an agent is a sequence of environment state and action pairs. We present an example trajectory $r$ of an agent below:

$$
r: s_{0} \stackrel{a_{1}}{\rightarrow} s_{1} \stackrel{a_{2}}{\rightarrow} \ldots \stackrel{a_{n}}{\longrightarrow} s_{n}
$$

Where $s_{0} \in S$ is the initial environment state of the agent, $a_{1} \in A$ is the action the agent took at $s_{0}, s_{1}$ is the environment state of the agent after performing the action $a_{1}$, and so on. The arrows here highlight how the action at an environment state may affect the next environment state of the agent.

In order to formalize the definition of an agent, we also need to define the notation we will use to represent the set of all finite trajectories. Therefore, we will notate the set of all finite trajectories with $\mathcal{R}$. Similarly, we will notate the subset of finite trajectories that end with a state $s \in S$ with $\mathcal{R}^{S}$, and the subset of finite trajectories that end with an action $a \in A$ with $\mathcal{R}^{A}$.

Before formalizing the definition of an agent, it is important to differentiate between the following types of agents: reactive agents, and state-based agents. A reactive agent is an agent that takes an action solely based on its current environment state, requiring no information about past environment states or actions [13]. Hence, we 
can formalize the definition of a reactive agent using the following function:

$$
\text { Ag } g_{\text {reactive }}: S \rightarrow A
$$

Here, the function $A g_{\text {reactive }}$ maps the set of environment states to the set of actions the agent can take.

On the other hand, a state-based agent considers past environment states and actions in addition to its current environment state to select which action to take [13]. Thus, the word "state" in state-based agent refers to its memory of past environment states and actions. For example, a state-based soccer agent that lost sight of the ball requires recalling the last environment state it saw the ball to determine which direction to turn in. Hence, we can formalize the definition of a state-based agent using the following function:

$$
\text { Ag } g_{\text {state-based }}: \mathcal{R}^{S} \rightarrow A
$$

Here, the function $A g_{\text {state-based }}$ maps the set of finite trajectories that end with an environment state to the set of actions.

We can also remodel a state-based agent by defining a finite set of hidden internal states $I$ which represent the memory of a state-based agent. For instance, a hidden internal state $i \in I$ would contain the relevant information from past environment states and actions that the agent uses to choose an action. Therefore, the function of 
a state-based agent can be remodelled using the following functions:

$$
\begin{array}{r}
F: \mathcal{R}^{A} \rightarrow I \\
A g_{\text {state-based }}: S \times I \rightarrow A
\end{array}
$$

Where $F$ is a function that maps a trajectory that ends with the previous action of the agent, $r \in \mathcal{R}^{A}$, to the current hidden internal state of the agent, $i_{t} \in I$. We can also remodel the function $F$ to be recursive:

$$
F_{\text {recursive }}: S \times A \times I \rightarrow I
$$

Here, the function $F_{\text {recursive }}$ maps the previous environment state $s_{t-1} \in S$, the previous action $a_{t-1} \in A$, and the previous hidden internal state $i_{t-1} \in I$ to the current hidden internal state of the agent $i_{t} \in I$.

Now that we have defined state-based and reactive agents, we will define the imitation learning problem in the context of imitating reactive agents in the next section. In the section that follows, we will define the imitation learning problem in the context of state-based agents.

\subsection{Imitating Reactive Experts}

Imitation learning involves training an agent to generalize/imitate the behavior of an expert using example trajectories of the expert, and possibly the feedback of the expert [5]. Here, the expert is simply another agent; thus, it can either be a reactive expert or a state-based expert. Moreover, an expert can be either a human or a computer program. We will assume that the environment state input of the expert and 
the agent are identical and represent the actual state of the environment. Therefore, in the case of reactive experts, the imitation learning problem involves learning the function in Equation (2.1).

Thus, imitating reactive experts can be easily reduced to a supervised learning problem that simple machine learning models can solve. For instance, a simple MultiLayer Perceptron $(M L P)$ can learn the mapping from the current environment state (input) to the action (output) of the reactive expert.

However, learning state-based behavior requires the use of special machine learning models and techniques due to the complexity of the function that it attempts to learn. In the next section, we will introduce the imitation learning problem of state-based experts.

\subsection{Imitating State-based Experts}

Imitation learning in the context of state-based experts involves learning the functions in Equation (2.3) and Equation (2.4). Therefore, an imitation learning agent that is imitating a state-based expert needs to learn two functions: a function that maps a trajectory that ends with an action to a hidden internal state and a function that maps the hidden internal state and the current environment state of the agent to an action.

Learning the first function, i.e. the function in Equation (2.3), is a difficult task since the internal state $i \in I$ of a state-based expert is not observable. Moreover, the trajectory of an agent is a sequence that is continuously increasing in length. Thus, traditional machine learning models such as MLP networks and traditional Case-Based Reasoning $(C B R)$ techniques are not capable of imitating state-based experts. This 
is because they cannot take inputs of varying length such as the trajectory of the agent. Moreover, they cannot learn the function in Equation (2.3) directly because the internal state of the expert is not observable.

However, Recurrent Neural Networks (RNN) are a perfect fit for imitating statebased experts since they can take sequential input of variable length. Moreover, they maintain a hidden state which is analogous to the hidden internal state of a state-based expert. In the next subsection, we will define RNNs and the architecture of a RNN; specifically, the architecture of a Long Short-Term Memory (LSTM) cell. We will also explain what makes RNNs a good fit for imitating state-based experts.

\subsubsection{Long Short-Term Memory (LSTM)}

Recurrent Neural Networks (RNN) are a class of neural networks that attempt to learn two functions: a function that maps the current input and the previous internal state (memory) of the RNN to the current internal state, and a function that maps the current input and the current internal state of the RNN to its output [23]. Hence, we can represent those two functions in the context of imitation learning using the following equations:

$$
\begin{aligned}
& H: S \times I \rightarrow I \\
& G: S \times I \rightarrow A
\end{aligned}
$$

Where $H$ is the function that maps the current environment state $s_{t} \in S$ and the previous internal state $i_{t-1} \in I$ of the RNN to its current internal state $i_{t} \in I$, and $G$ is the function that maps the current environment state $s_{t} \in S$ and the current internal state $i_{t} \in I$ of the RNN to its current action $a_{t} \in A$. Therefore, we can 
rewrite Equation (2.6) and Equation (2.7) as the following equations:

$$
\begin{aligned}
& i_{t}=H\left(s_{t}, i_{t-1}\right) \\
& a_{t}=G\left(s_{t}, i_{t}\right)
\end{aligned}
$$

One can see how the function $H$ in Equation (2.6) is analogous to the function $F$ in Equation (2.5); however, the function $H$ does not include the previous action $a_{t-1} \in A$ in its parameters. We can also see how the function $G$ in Equation (2.7) is analogous to the function $A g_{\text {state-based }}$ in Equation (2.4). This makes RNN networks suitable for imitating state-based experts. In Figure 2.1, we present an example RNN chain.

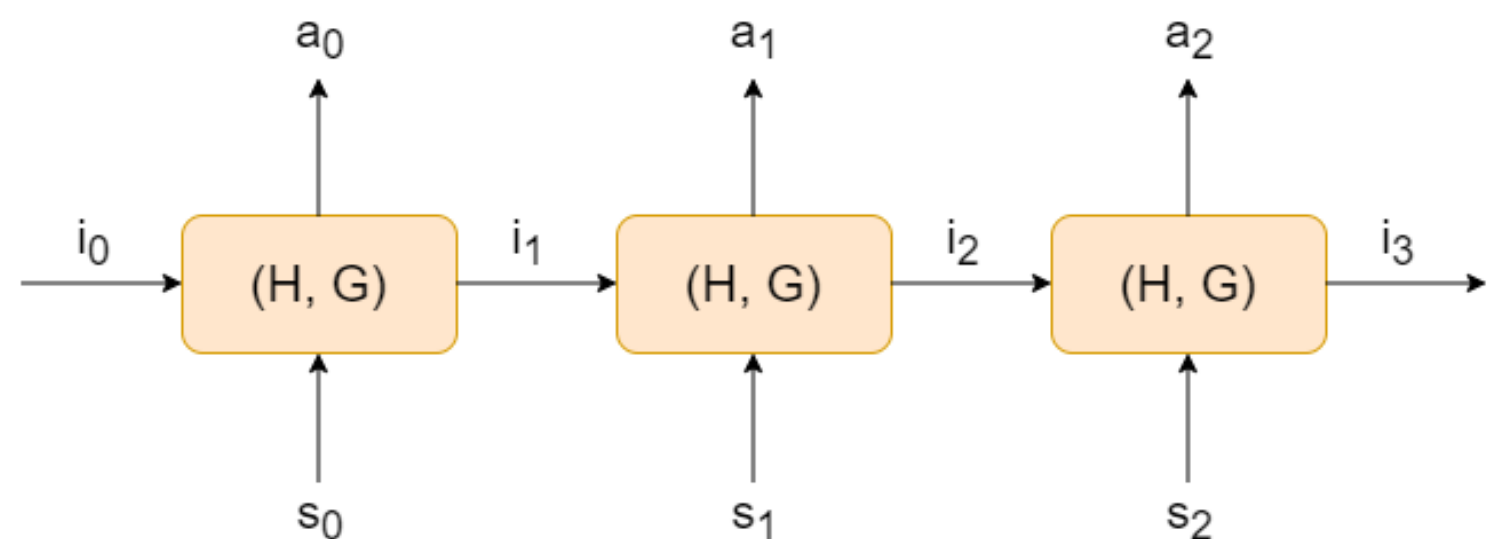

Figure 2.1: An example RNN chain. Here, we can see how each internal state is updated based on the current environment state $s_{t} \in S$ and the previous internal state $i_{t-1} \in I$. An action $a_{t} \in A$ is taken at each time step $t$. The cells highlighted in orange represent an RNN cell unrolled through time.

Different types of RNNs have different parametrical forms of their functions $H$ and $G$. Therefore, each RNN version has a different cell design. For instance, vanilla $R N N[24]$ is the first version of RNNs and we present its cell in Figure 2.2. 


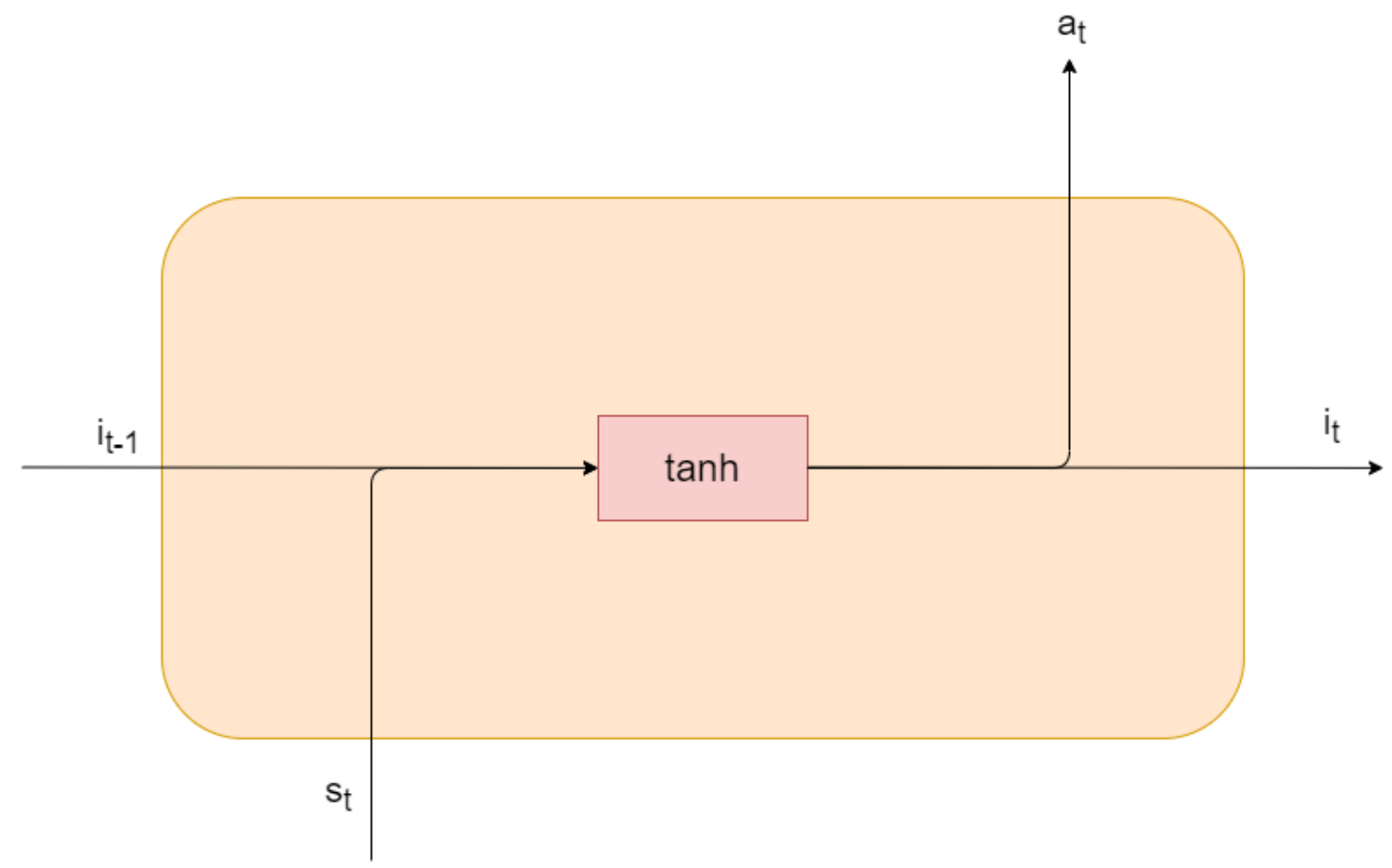

Figure 2.2: The vanilla RNN cell. Here, we indicate that the tanh function contains parameters that are learnt by enclosing it in a red box. $i_{t}$ and $a_{t}$ are computed using the functions in Equation (2.10) and Equation (2.11).

$$
\begin{aligned}
& i_{t}=\tanh \left(W s_{t}+U i_{t-1}+b\right) \\
& a_{t}=V i_{t}+c
\end{aligned}
$$

The problem with the vanilla RNN cell is that it suffers from a gradient vanishing/exploding problem [14]. The gradient vanishing/exploding problem refers to the parameters of the vanilla RNN not being properly updated as we perform backpropagation through time to update its parameters. Specifically, the gradient vanishing problem is observed when the training sequence is long due to the gradient of the tanh function being less than 1. On the other hand, the gradient exploding issue is observed when the parameter $W$ of the vanilla RNN is large enough to overcome the 
gradient of tanh [14].

To overcome this issue, Sepp and Jürgen [14] and Gers [25] proposed the LSTM cell. We present the LSTM cell proposed by Gers [25] in Figure 2.3. The red line in the diagram has no learnt parameters along its path to create a "gradient freeway" which mitigates the gradient vanishing/exploding problem.

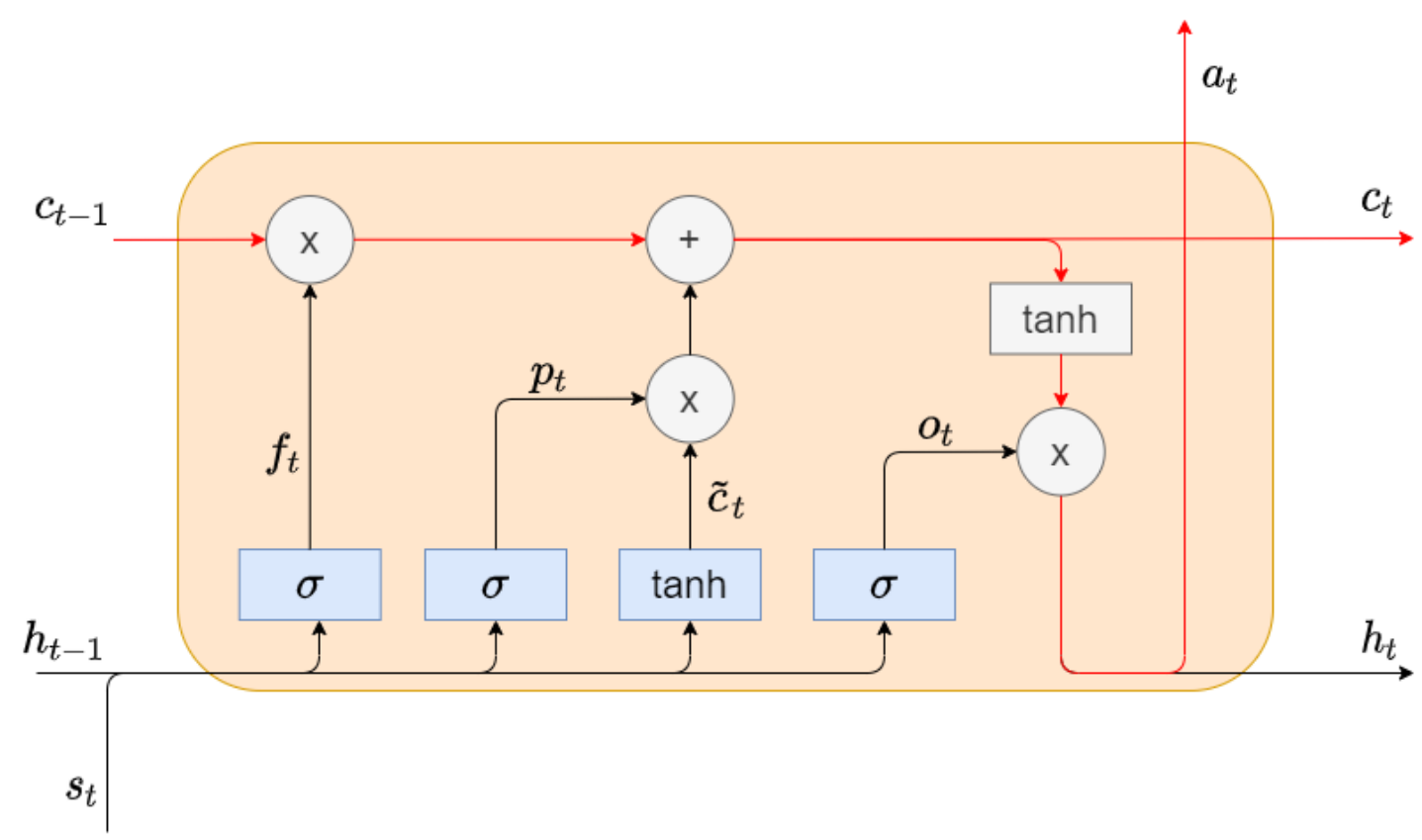

Figure 2.3: The LSTM cell. Here, the functions that contain parameters that are learnt by enclosed in a blue box. Functions that do not contain learnt parameters are contained in a grey box/circle. The red arrows here represent the "gradient freeway" of the LSTM cell that addresses the gradient vanishing/exploding problem of vanilla RNN.

An LSTM cell consists of 4 gates, represented in the diagram in Figure 2.3 by a red box each. The first gate from the left is the forget gate that outputs $f_{t}$. The second gate is the input gate signal that outputs $p_{t}$. It is responsible for selecting the new information to store in the control line. The third gate is respz'onsible for creating new information $\widetilde{c}_{t}$. Lastly, the fourth gate is the output gate signal $o_{t}$ that selects 
which data to keep as output $h_{t}$ and $a_{t}$. The hidden internal state of the LSTM is the concatenation of the "data line" $c_{t}$ and the "control line" $h_{t}$; that is, $i_{t}=\left(c_{t}, h_{t}\right)$. The equation of each signal is presented in Equations (2.12) to (2.17).

$$
\begin{aligned}
f_{t} & =\sigma\left(W^{f} s_{t}+U^{f} h_{t-1}+b^{f}\right) \\
p_{t} & =\sigma\left(W^{p} s_{t}+U^{p} h_{t-1}+b^{p}\right) \\
\widetilde{c}_{t} & =\tanh \left(W^{\widetilde{c}} x_{t}+U^{\widetilde{c}} h_{t-1}+b^{\widetilde{c}}\right) \\
o_{t} & =\sigma\left(W^{o} s_{t}+U^{o} h_{t-1}+b^{o}\right) \\
c_{t} & =p_{t} \odot \widetilde{c}_{t}+f_{t} \odot c_{t-1} \\
h_{t}=a_{t} & =o_{t} \odot \tanh \left(c_{t}\right)
\end{aligned}
$$

Despite the LSTM cell being well suited for state-based imitation learning, there is still another issue that it does not address. While most application domains, such as Natural Language Processing (NLP), that make use of LSTM do not have outputs that affect the next input of the LSTM; imitation learning agents affect their environment state (input) with their action (output). Hence, if a trained LSTM imitation learning agent is not imitating the expert perfectly, the environment state distribution it will experience in the environment will differ from the environment state distribution it was trained on. Hence, it will not imitate the expert adequately as its environment state distribution will drift away from the environment state distribution of the expert. In the next section, we will present the problem introduced by imitation learning and a solution to address this problem. 


\subsection{Dealing With the Dependency of Environment States on Actions}

As we mentioned in the previous section, the environment state of an agent is dependent on its actions in most domains. For instance, the next environment state of a soccer agent is dependent on the current environment state and the action the agent took, such as kicking the ball or turning in a direction. Hence, the environment state distribution of an agent violates the i.i.d. assumption made in traditional statistical learning methods. Once the trained agent mistakenly selects an action that the expert would not select at an environment state $s$, the environment state distribution that the agent visits is no longer identical to the environment state distribution that the agent was trained on. This results in the behavior of the trained agent further diverging from the behaviour of the expert. In this section we will present Dataset Aggregation (DAgger), an algorithm proposed by Ross et al. [21], to address this issue during training.

\subsubsection{Dataset Aggregation (DAgger)}

DAgger, short for Dataset Aggregation, is an iterative algorithm that trains an agent to imitate an expert under the distribution of the policy's visited environment states as opposed to the expert's visited environment states [21]. We describe the DAgger algorithm in Algorithm 1.

The DAgger algorithm requires an expert $A g^{*}: S \rightarrow A$, and a hyper-parameter $\beta$. Before the first iteration, the algorithm initializes the parameters of the initial imitation learning agent $\hat{A} g_{1}$. Next, it creates a new agent $A g_{i}$ that mixes the two

functions, $\hat{A} g_{i}$ of the imitation learning agent and $A g^{*}$ of the expert. The new agent 
$A g_{i}$ uses the imitation learning agent $\hat{A g}$ to select its actions a fraction of the time and the expert $A g^{*}$ to select its actions for another fraction dependent on its hyperparameter $\beta_{i}$. This new agent is then executed in the environment for $T$ steps to create a trajectory. The expert $A g^{*}$ is then used to replace any incorrect actions in the new $T$-step trajectory with the correct action and then, the corrected trajectory is added to the dataset. The next imitation learning agent $\hat{A} g_{i+1}$ is then trained on the new dataset. This process is repeated for a fixed number of iterations and the best performing $\hat{A} g_{i}$ is returned as the final trained imitation learning agent.

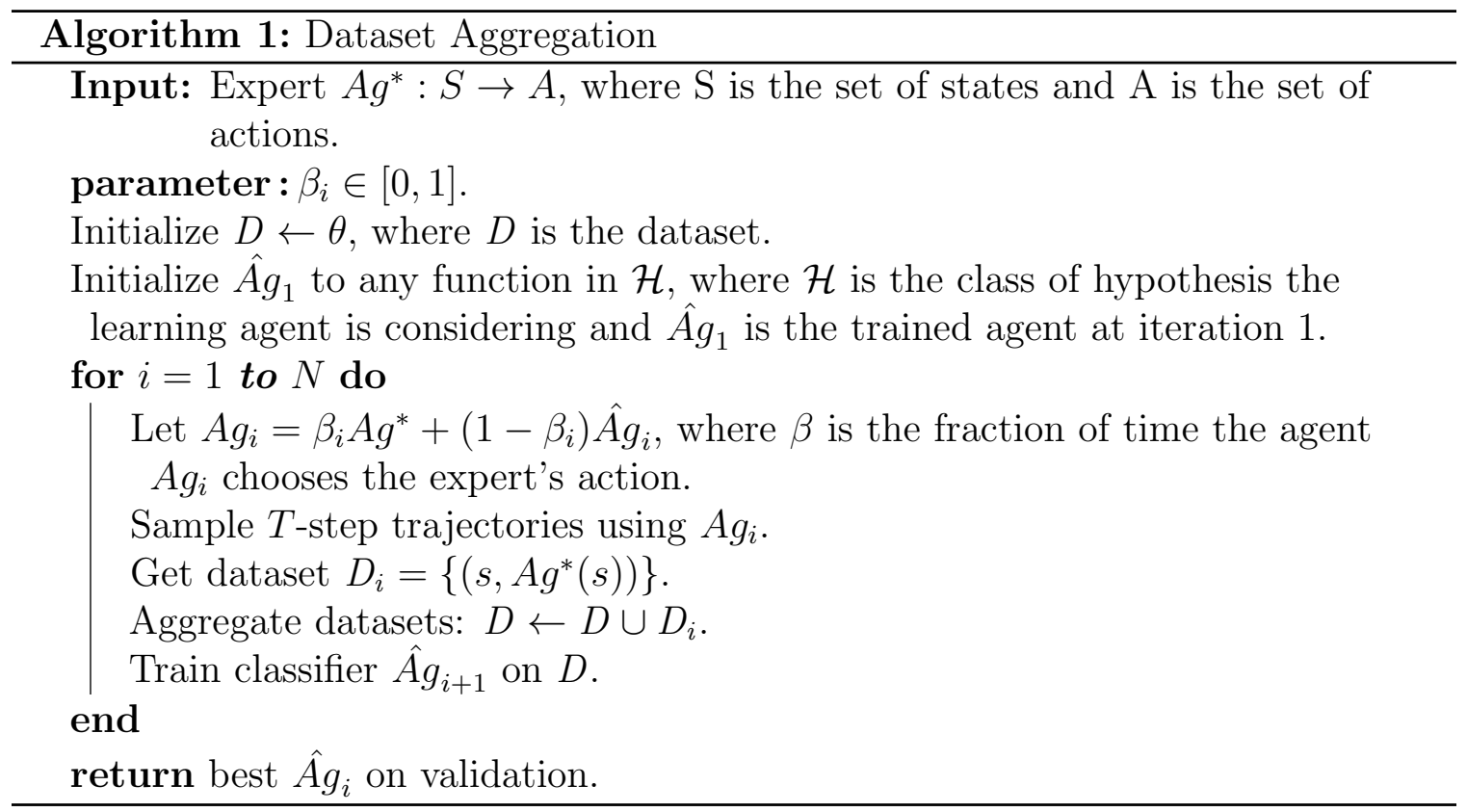

Therefore, the final result of DAgger is an imitation learning agent $\hat{A} g_{i}$ that is trained on its own environment state distribution, rather than the environment state distribution of the expert. Thus, imitation learning agents trained using DAgger should perform much better than those that are trained using a dataset that is collected using the expert alone. However, DAgger was clearly meant to be used to imitate reactive agents; hence, the agent's function in the DAgger algorithm is mapping the 
set of environment states to the set of actions. We will make use of this method in our thesis to see if it also improves the performance of state-based imitation learning techniques at imitating state-based experts.

\subsection{Conclusion}

In this chapter, we have introduced all the necessary background required to understand the upcoming chapters. We discussed the difference between a reactive agent and a state-based agent, and we discussed what imitating each type of agent entails. We also discussed how we need to use special machine learning techniques when imitating state-based experts as they require learning a function that maps a sequence to an output instead of a function that maps a fixed input to an output. Moreover, we learnt about RNNs and why LSTM is a good fit for imitating state-based behavior. We also described DAgger and how it helps mitigate the i.i.d. assumption of machine learning models when training imitation learning agents. However, we saw how DAgger was designed to be used to imitate reactive agents using techniques that only take the current environment state as input. In the next chapter, we will present state-of-the-art research in imitation learning that make use of the background we presented in this chapter. 


\section{Chapter 3}

\section{Related Work}

\subsection{Introduction}

In the context of state-based imitation learning, there has been a lot of research focused on proposing new techniques and evaluating which techniques work best at imitating state-based behavior in many domains $[20,1,3,2,4]$. In this chapter, we will discuss the benchmarks that are commonly used in the field of state-based imitation learning, the different techniques employed to learn state-based behavior, the different testing methodologies adopted to evaluate those techniques, and lastly, the metrics used to compare them. We will see how current testing methodologies adopted in the field of state-based imitation learning do not always provide us with reliable results and how LSTM networks were never compared to state-of-the-art techniques of state-based imitation learning. 


\subsection{Benchmarks}

\subsubsection{Introduction}

In this section, we will discuss two domains commonly used to evaluate imitation learning agents: the discrete vacuum cleaner domain [26] and the continuous RoboCup soccer domain [27]. We will study the environment and action space, and the experts commonly used in each domain to evaluate state-based imitation learning techniques.

\subsubsection{Vacuum Cleaner Domain}

\subsubsection{Introduction}

The vacuum cleaner domain is a simplified simulation of a Roomba: an autonomous robotic vacuum cleaner that utilizes sensors and two wheels that operate independently to avoid obstacles and navigate in a room [26]. The simulation's environment consists of a rectangular grid of cells that are either empty, contain an obstacle, or contain dirt. The agent in the simulation is a vacuum cleaner that can move one pixel in the right, left, up, or down directions, or it can stay still. In the case where an agent tries to move in the direction of an obstacle, the agent stays still. On the other hand, when a direction contains dirt, once the agent moves on top of that pixel, the pixel no longer contains dirt.

It is common for a study to model the environment state vector of the domain depending on the behavior its experts exhibit. For instance, the study of Tîrnăucă et al. [3] has no experts that take dirt into consideration; thus, they model the vacuum cleaner environment using an environment state vector that excludes information about dirt. Whereas, the study of Ontañón et al. [1] have experts that consider dirt in their 
environment; hence, they use an environment state vector that includes information about dirt. Therefore, we will introduce the 2 different models of the vacuum cleaner environment used in the studies we will discuss in this chapter. We will also discuss the different experts used in each model of the vacuum cleaner environment.

\subsubsection{Vacuum Cleaner With Dirt}

The experts used in the vacuum cleaner domain in the studies of Ontañón et al. [1] and Gunaratne et al. [2] consider the presence of dirt when deciding to move in a direction or to stand still. Hence, it is important that their model of the vacuum cleaner environment contains information on whether a pixel contains dirt or not. Thus, they model the environment vector of the vacuum cleaner domain with 8 categorical variables, 2 for each direction (north, east, west, and south): one variable is set to 1 if there is dirt in the respective direction, or to 0 if there is an obstacle in the respective direction; the other variable is set to 1 if the obstacle/dirt is far (i.e. not in the adjacent pixel in the respective direction), or to 0 if it is close.

The experts that Ontañón et al. [1] and Gunaratne et al. [2] attempted to imitate are listed in Table 3.1, and the expert that was only used by Gunaratne et al. [2] is described in Table 3.2. Recall from Chapter 2 that a reactive agent is one that only considers the current environment state. Whereas, a state-based agent is one that considers past environment state(s) or action(s) in addition to the current environment state. Here, the Smart Random expert only considers the current environment state; hence, it was designed to test the performance of a technique at imitating reactive behavior. On the other hand, the Smart Straight Line, ZigZag, Fixed Sequence, Smart Explorer, and Frequency experts use information from past environment state(s) or action(s) to test a techniques ability at imitating different types of state-based 
behavior.

The Smart Straight Line expert tests the ability of a technique to imitate behavior that requires recalling the previous action in addition to considering the current environment state. The ZigZag expert was designed to test a technique's capability at imitating behavior that requires recalling the previous action, and identifying if a certain environment state was visited in the past (i.e. a bottom corner). The purpose of the Fixed Sequence expert is to evaluate the ability of a technique to imitate behavior that only considers its past actions when selecting the next action. The Smart Explorer expert is used to test how well a technique can imitate behavior that requires recalling all previous states and actions. Lastly, the Frequency expert was designed to test the ability of a technique to imitate state-based behavior that does not directly considers past environment states and actions; instead, it considers the frequency of environment states that the expert visited along its path. 


\begin{tabular}{|c|c|}
\hline Expert & Description \\
\hline Smart Random & $\begin{array}{l}\text { The vacuum cleaner randomly selects } \\
\text { the next direction from the set of } \\
\text { available directions at the current } \\
\text { environment state, unless there is dirt in } \\
\text { one direction, in which case it will select } \\
\text { the direction of the dirt. }\end{array}$ \\
\hline Smart Straight Line & $\begin{array}{l}\text { The vacuum cleaner selects a random } \\
\text { valid direction and always moves in the } \\
\text { same direction as the previous direction; } \\
\text { when it reaches an obstacle, it repeats } \\
\text { this behavior. If there is dirt in one } \\
\text { direction, it will move in that direction } \\
\text { instead. }\end{array}$ \\
\hline ZigZag & $\begin{array}{l}\text { The vacuum cleaner moves right till it } \\
\text { reaches an obstacle; when an obstacle is } \\
\text { encountered, it moves down once. It } \\
\text { then alternates to moving left (in a } \\
\text { zig-zag fashion) till it reaches another } \\
\text { obstacle. It continues repeating this } \\
\text { pattern till it can no longer go down. At } \\
\text { this point, it repeats the pattern in the } \\
\text { upwards direction and so on. }\end{array}$ \\
\hline Fixed Sequence & $\begin{array}{l}\text { The vacuum cleaner repeats a fixed } \\
\text { sequence of actions ( } 21 \text { actions long) } \\
\text { regardless of the environment state. }\end{array}$ \\
\hline Smart Explorer & $\begin{array}{l}\text { The vacuum cleaner agent remembers } \\
\text { all the pixels that it has visited. It } \\
\text { selects the action that will lead it to a } \\
\text { pixel that it has not visited before with } \\
\text { a probability of } 0.75 \text {. If it sees dirt in a } \\
\text { direction, it will move towards that } \\
\text { direction. Once the entire grid is } \\
\text { explored the agent stops. }\end{array}$ \\
\hline
\end{tabular}

Table 3.1: The experts used in the model of the vacuum cleaner domain that includes dirt. Those experts were used in the work of Ontañón et al. [1] and some of them were used in the work Gunaratne et al. [2] 


\begin{tabular}{ll}
\hline Expert & Description \\
\hline & The vacuum cleaner moves downwards \\
& and records the frequency of \\
& environment states that contained dirt \\
& in the west direction and the frequency \\
& of environment states that had a wall in \\
& the west or east direction. If the \\
& frequency of dirt to the west is larger \\
& than the frequency of walls to the west \\
& and east of the agent when it reaches an \\
& obstacle, it moves west, otherwise, it \\
& moves east. It then continues moving \\
& upwards, and repeats this behavior. \\
& This expert was only used in the study \\
& of Gunaratne et al. $[2]$ \\
\hline
\end{tabular}

Table 3.2: The expert used in the study of Gunaratne et al. [2] This expert was used in the vacuum cleaner with dirt model. 


\subsubsection{Vacuum Cleaner Without Dirt}

In the work of Tîrnăucă et al. [3], the authors created maps of the vacuum cleaner domain that do not contain cells with dirt. Instead their map consists of cells that are either empty or contain an obstacle. Consequently, the experts that they use in their experiments do not care about the existence of dirt in the direction they select to move in. Therefore, they model the vacuum cleaner domain using 4 categorical variables that indicate whether an obstacle exists in the adjacent pixel (1), or not (0) in the respective direction.

We describe the experts used in the study of Tîrnăucă et al. [3] in Table 3.3. Some of the experts in Table 3.3 are simplified versions of the experts that consider dirt in Table 3.1. The experts of this study were designed to include an expert that exhibits reactive behavior (WALK), and two experts that exhibit state-based behavior (CRASH and ZIGZAG_NO_DIRT). The CRASH agent selects its action based on the current environment state and the previous action. On the other hand, the ZIGZAG expert selects its next action based on the previous action, current environment state, and whether it previously reached a right corner or not (past environment state). 


\begin{tabular}{|c|c|}
\hline Expert & Description \\
\hline WALK & $\begin{array}{l}\text { The vacuum cleaner always moves in } \\
\text { the same (valid) direction depending on } \\
\text { the current environment state. }\end{array}$ \\
\hline RND_WALK & $\begin{array}{l}\text { The vacuum cleaner randomly selects } \\
\text { the next direction from the set of } \\
\text { available directions at the current } \\
\text { environment state. }\end{array}$ \\
\hline CRASH & $\begin{array}{l}\text { The vacuum cleaner always takes the } \\
\text { same direction as the previous direction; } \\
\text { when it reaches an obstacle, it selects a } \\
\text { predefined direction based on the } \\
\text { obstacle's position. }\end{array}$ \\
\hline RND_CRASH & $\begin{array}{l}\text { Same as the CRASH expert, however, } \\
\text { once the vacuum cleaner reaches an } \\
\text { obstacle, it randomly selects a direction } \\
\text { from the set of available directions. }\end{array}$ \\
\hline ZIGZAG_NO_DIRT & $\begin{array}{l}\text { The vacuum cleaner moves up till it } \\
\text { reaches an obstacle; when an obstacle is } \\
\text { encountered, the next direction is right. } \\
\text { It then alternates to moving downwards } \\
\text { (in a zig-zag fashion) till it reaches } \\
\text { another obstacle. It continues repeating } \\
\text { the pattern till it reaches a right corner, } \\
\text { when its internal state is updated and it } \\
\text { moves left when an obstacle is } \\
\text { encountered (instead of right). This } \\
\text { behavior depends on the current } \\
\text { environment state, the internal state, } \\
\text { and the previous action. }\end{array}$ \\
\hline RND_ZIGZAG_NO_DIRT & $\begin{array}{l}\text { Same as the previous strategy, however, } \\
\text { when the vacuum cleaner reaches a } \\
\text { corner, it may/may not change its } \\
\text { internal state. }\end{array}$ \\
\hline
\end{tabular}

Table 3.3: The experts used in the work of Tîrnăucă et al. [3] with the vacuum cleaner model that excludes dirt. 


\subsubsection{Conclusion}

All the techniques we will discuss in this chapter have been previously evaluated in the vacuum cleaner cleaner domain. Specifically, the vacuum cleaner domain with dirt model has been used to test all techniques except for Probabilistic Finite Automata (PFA) [3]. However, PFA were tested using the vacuum cleaner without dirt model in the study of Tîrnăucă et al. [3].

The experts that were used in this domain test a large variety of state-based behavior. The vacuum cleaner domain has a discrete environment space; hence, techniques that are limited to discrete environment spaces can be used in this domain. This makes the vacuum cleaner domain a benchmark where all techniques can be evaluated.

\subsubsection{RoboCup Soccer Domain}

\subsubsection{Introduction}

The RoboCup soccer simulation is a $2 \mathrm{D}$ soccer simulation developed by RoboCup: an organization with the objective of having a team of fully autonomous humanoid robot soccer players that comply with the official rules of FIFA and are capable of defeating the winners of the most recent World Cup by 2050 [27]. RoboCup currently hosts a multitude of leagues; one of which is the RoboCupSoccer league, that challenges researchers to develop a competitive team of soccer agents. This league is further divided into multiple sub-leagues, that range from developing a team of simulated 2D soccer agents to a team of robotic humanoid soccer agents.

The soccer simulation is a partially-observable continuous domain where the agent is a soccer player in a soccer field. The agent can only observe objects that are within 
its field of vision. The ball, the enemy goal, its own goal, and other players in the field are some examples of the objects that exist in the simulation. The agent can choose to kick in a chosen direction, dash with a certain power, or turn with a certain angle relative to the direction it is aligned in. The RoboCup soccer simulation server provides each agent with information about the direction and distance of each object in its field of view. This information is encoded as a string that contains the features of each object; hence, it is up to the user to encode the environment of the agent depending on the imitation learning technique the user employs. We will describe the RoboCup soccer model of Gunaratne et al. [4] and the RoboCup soccer experts they designed in Section 3.2.3.2.

\subsubsection{Discretized RoboCup Soccer Model}

Gunaratne et al. [4] make use of techniques that are limited to discrete domains in their study. Thus, they needed to discretize the RoboCup soccer environment into 3 binary variables that their experts consider when deciding which action to take: a variable indicating whether the ball is seen or not, another indicating whether the goal is seen or not, and lastly a variable indicating whether the ball is close or not.

They also discretize the action space into 3 actions: kick, dash, and turn that have

hard-coded parameters which depend on the direction of the goal and the direction of the ball. The authors use two experts: a reactive expert called Krislet and a statebased expert called KickIfLastDashed described in Table 3.4. The KickIfLastDashed expert tests the ability of a technique to imitate state-based behavior that rely on recalling the previous action. 


\begin{tabular}{ll}
\hline Expert & Description \\
\hline & The agent turns in the positive direction \\
& if the ball is not in the vision of the \\
& expert and if it is, it turns towards the \\
& ball. Once it is aligned with the ball, it \\
& dashes to the ball till the ball is in \\
& kicking range. If the goal is not in the \\
& vision of the expert when it is in kick \\
& range, it turns in the positive direction \\
Krislet & till it can see the goal. Once it sees the \\
& goal and the ball is close it kicks the \\
& ball to the goal. \\
& The agent exhibits the same behavior as \\
& Krislet. However, when it sees the goal \\
& and it is close to the ball, it kicks if the \\
& last action was a dash, otherwise, it \\
& performs a turn action. \\
\hline
\end{tabular}

Table 3.4: The experts used in the study of Gunaratne et al. [4] using the discretized model of RoboCup soccer.

\subsubsection{Conclusion}

The RoboCup soccer domain provides a benchmark to test the performance of imitation learning techniques in a partially observable domain. However, the study that compared many of the techniques that we will discuss later in this chapter discretized the environment space of RoboCup [4]; which eliminated the interesting properties of the domain. As such, we will remodel the environment state vector of this domain later in our thesis to maintain the properties of the domain and use it as a benchmark to test imitation learning techniques in a continuous, partially observable domain. 


\subsubsection{Conclusion}

In this section, we discussed the domains and experts used in previous studies to compare state-based imitation learning techniques. The vacuum cleaner domain provides a good variety of experts that consider different aspects of the run (whether it is previous actions, environment states, or the frequency of visited environment states). Moreover, it is well suited to test techniques that are limited to discrete domains. On the other hand, the RoboCup soccer domain provides an environment where we can test the limits of techniques that are not limited to discrete domains. In addition, it contains a large repository of experts that were used in the RoboCupSoccer simulation leagues. Thus, it is important to assess imitation learning techniques in this domain as they can be used to imitate real experts in the future. However, the RoboCup soccer environment was discretized by the study which compared state-based imitation learning techniques as some of the techniques were limited to discrete domains. Hence, the study did not capitalize on the complex nature of the domain. It also did not provide a wide variety of state-based experts in the RoboCup soccer domain. In the next section, we will discuss state-of-the-art state-based imitation learning techniques that were used in the studies we mentioned.

\subsection{Techniques}

\subsubsection{Introduction}

In this section, we will discuss the techniques described in the literature of state-based imitation learning. We will learn the limitations of each technique and the situations where each technique is preferred. The first method we will study is Temporal 
Backtracking (TB), a Case-Based Reasoning (CBR) based technique of imitating state-based behavior proposed by Floyd and Esfandiari in [20]. Next, we will discuss Dynamic Bayesian Networks (DBN) that are capable of learning state-behavior used in the work of Ontañón et al. [1] Later, we discuss a method that makes use of Probabilistic Finite Automata (PFA) to learn state-based behavior [3]. Lastly, we will learn about the similarity metrics proposed by Gunaratne et al. in [2] that enable CBR techniques to imitate state-based behavior.

\subsubsection{Temporal Backtracking}

Temporal Backtracking (TB) is a Case-Based Reasoning (CBR) approach that considers the trajectory of the agent as well as the trajectory of the expert when comparing the current environment state of the agent to its training set. TB was introduced by Floyd and Esfandiari [20] to enable CBR to imitate state-based behavior. The algorithm looks for the nearest neighbors of the current environment state and if it finds disagreeing actions, it recursively backtracks comparing the previous action or environment state of the nearest neighbors with that of the current trajectory, till it reduces to a non-disagreeing set of nearest neighbors. This allows the agent to learn behavior that may depend on past actions or environment states, with a bias towards more recent environment states and actions. 


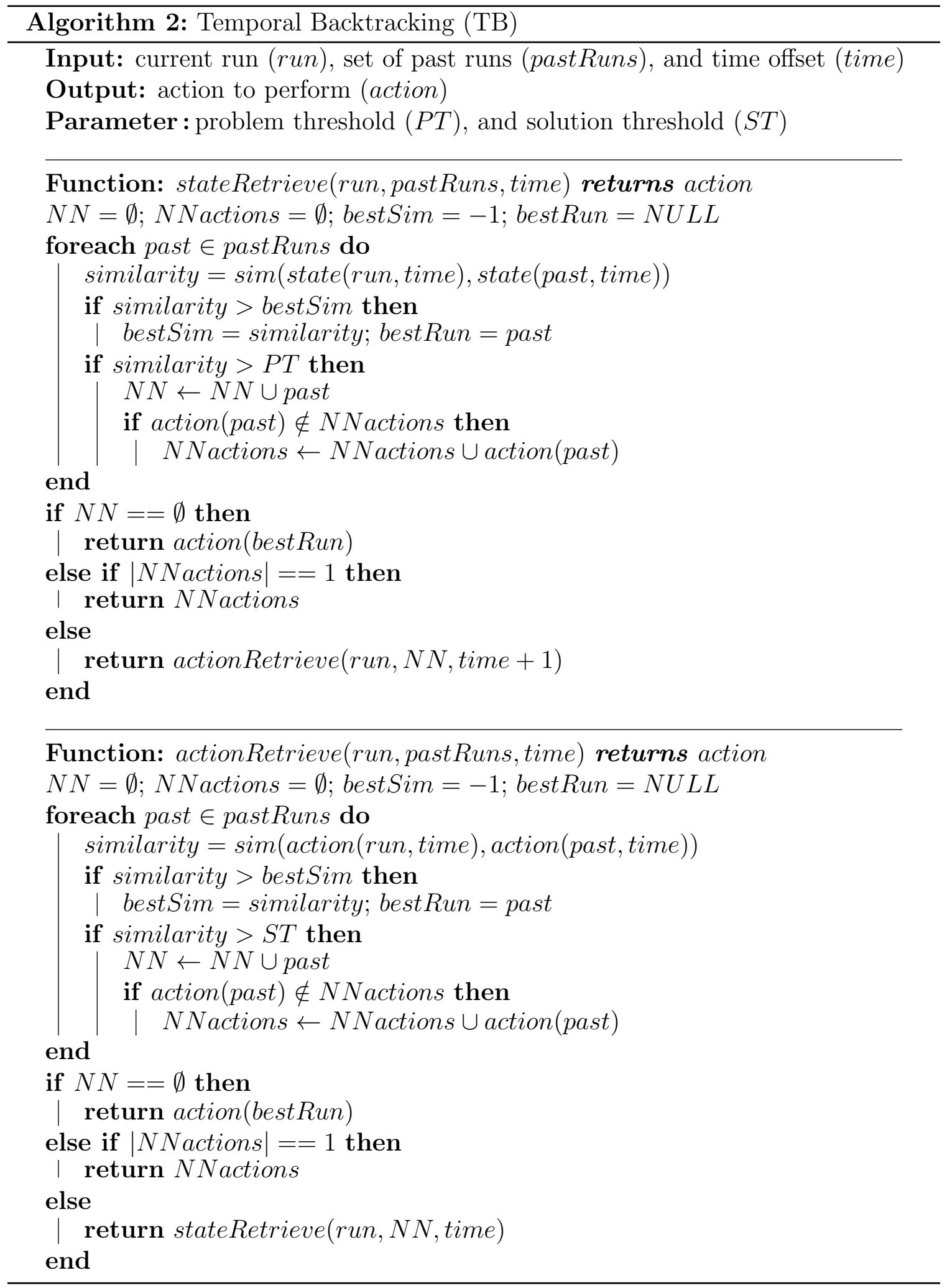


The TB algorithm is described in more detail in Algorithm 2. The stateRetrieve function of the algorithm is called with an initial time offset of 0 for the algorithm to consider the most recent environment state. The algorithm has two hyper-parameters: the problem threshold $(P T)$ and the solution threshold $(S T)$. The hyper-parameter PT marks the threshold beyond which the similarity between the environment state of the current run and the past run is considered close enough to be a neighbor of the current run. Similarly, ST marks the threshold beyond which the similarity between the action of the current run and the past run is considered close enough to be a neighbor of the current run. The functions action(run,time) and state(run,time) retrieve the action and environment state of the run at the selected time respectively. Lastly, the function action(run) retrieves the action selected at time offset 0 of the run.

There has been a lot of studies that compared this technique to the majority of the techniques that we will mention later in this chapter $[4,2]$. This algorithm has remained a solid benchmark that is used to compare new techniques that imitate state-based behavior till today. It is ideal in situations where the training set is not too large, as it is a lazy learning technique. However, its training set can be undersampled to optimize its run-time performance. Research has shown that although TB outperforms Dynamic Bayesian Networks (DBN), it may under-perform when imitating state-based experts that exhibit certain types of behavior in comparison to specialized CBR similarity metrics that we will discuss later in this chapter [2]. For example, TB struggles to imitate behaviors that involve repeating a fixed sequence of actions regardless of the environment state, and behaviors that rely on remembering the frequency of past environment states (the Fixed Sequence and Frequency experts described in Section 3.2.2.2). 
Recall from Chapter 2, that a state-based agent is defined using the function $A g: \mathcal{R}^{S} \rightarrow A$, where $\mathcal{R}^{S}$ is the set of runs that end with an environment state. We also recall that this function can be simplified to $A g: S \times I \rightarrow A$, where $I$ is the set of the agent's hidden internal states. This implies that there exists a function

$F: \mathcal{R}^{A} \rightarrow I$, that the agent uses to retrieve its next hidden internal state, where $\mathcal{R}^{A}$ is the set of runs that end with an action. Therefore, the function $F$ must also be learnt by the imitation learning technique in order for the technique to be universal. However, TB makes two assumptions on the function $F$. It assumes that the agent's hidden internal state is dependent on the most recent environment states and actions, and that the features of the environment states and actions are the salient information that decide the hidden internal state of the agent. However, this is not always the case. For instance, the Frequency expert's hidden internal state is dependent on the frequency of past environment states and not a certain feature(s) in recent environment states. Hence, TB under-performs when imitating this expert and other experts that do not follow its assumptions, and this is evident in the results of the studies we discussed.

Despite this, TB is a well-rounded technique supported by a solid concept that makes it ideal for imitating most state-based experts, assuming they follow its assumptions. Moreover, it is not limited to discrete domains; hence, it can be used in more complex domains such as the continuous RoboCup soccer domain. Nonetheless, there is a need to propose techniques that make no assumption on the function $F$, which maps a run that ends with an action to a hidden internal state.

\subsubsection{Dynamic Bayesian Networks}

A Bayesian Network $(B N)$ is a probabilistic graphical model that represents the conditional dependencies between a set of random variables in a directed acyclic 
graph [28]. In the context of imitation learning, this model be used to represent the conditional probability $p(A \mid S)$, where $A$ is the set of actions and $S$ is the set of environment states. The structure of a typical BN used to imitate an expert is presented in Figure 3.1.

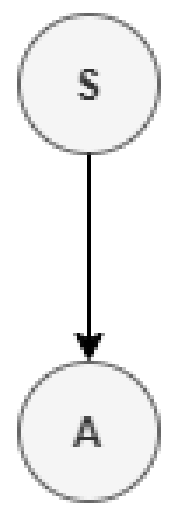

Figure 3.1: An example Bayesian Network where the action $A$ is dependent upon the environment state $S$. Note that $S$ and $A$ can be multi-dimensional.

BNs are trained by observing an expert performing a task in the environment and learning $p(A \mid S)$ from the example trajectories of the expert. However, a limitation of the standard BN depicted in Figure 3.1 is that it is not capable of learning state-based behavior as the actions are only dependent on the current observed state of the environment and the environment state is assumed to be independent of the previous environment state and the previous action the agent took. Therefore, the structure of the BN needs to be adjusted in order to learn state-based behavior.

Ontañón et al. [1] introduced Learning from Observation Dynamic Bayesian Network (LfODBN): a Dynamic Bayesian Network (DBN) that is capable of learning state-based behavior. A DBN is a BN that consists of a series of identical time-slices where the nodes in each slice can only depend on nodes in the same time slice or the previous time slice [29]. This allows their model to select actions based on information from the past. The model of their proposed LfODBN is presented in Figure 3.2. The 
difference between their proposed model and an Input-Output Hidden Markov Model (IOHMM) [30] is that it has a dependency between the previous action $A_{t-1}$ and the hidden internal state node $I_{t}$. An IOHMM has no dependency between the internal state and the previous action (refer to Figure 3.2 and Figure 3.3).

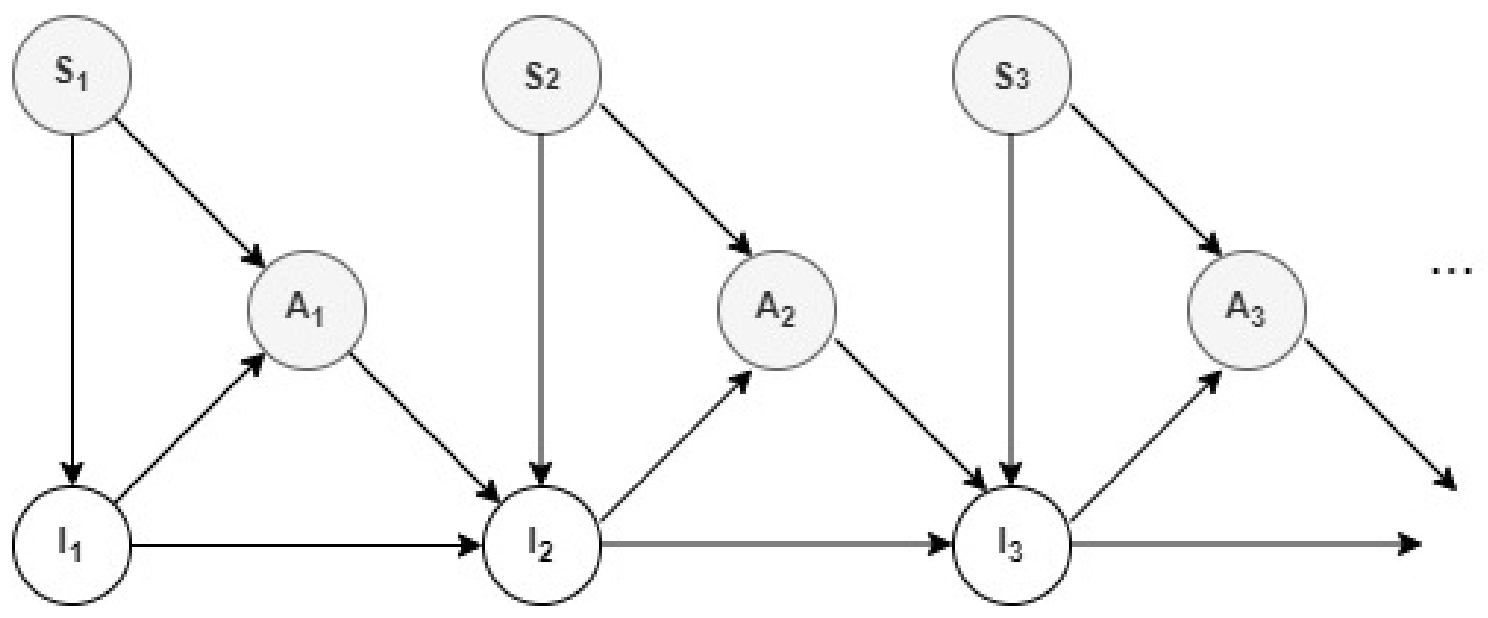

Figure 3.2: LfODBN Model. $S_{t}$ is the environment state, $A_{t}$ is the action, and $I_{t}$ is the hidden internal state of the agent.

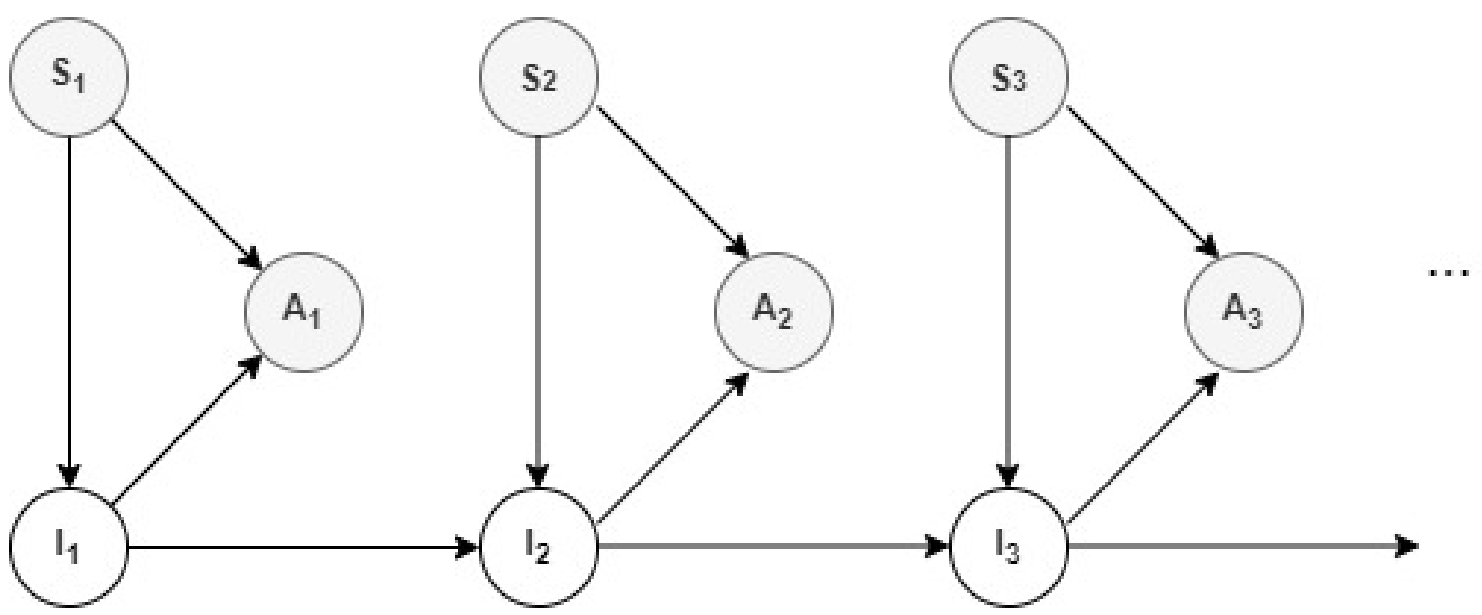

Figure 3.3: Input-Output Hidden Markov Model. Note how the hidden internal state node does not depend on the previous action.

The hidden internal state nodes in the LfODBN model are meant to represent the hidden internal state of a state-based expert. Since the hidden state of the expert 
is unobserved, this model requires the use of the Expectation-Maximization (EM) algorithm [31] in order to learn the parameters of the LfODBN.

Ontañón et al. [1] evaluated their LfODBN model in the discrete vacuum cleaner domain we mentioned earlier in this chapter. They compared their proposed model to IOHMM at imitating the state-based experts described in Section 3.2.2.2. Their results indicated that the LfODBN outperformed the IOHMM at imitating all their state-based experts.

Later work re-evaluated LfODBNs against IOHMMs and TB in both the vacuum cleaner domain and the RoboCup soccer domain [4]. Their reproduced results confirmed that LfODBN outperformed or matched the IOHMM performance in both domains when imitating state-based experts. However, they found that TB outclassed both techniques at imitating all the state-based experts that they used in both domains.

In conclusion, the LfODBN seems to outperform its IOHMM counterpart at imitating state-based experts in both domains. Thus, in cases where CBR techniques are not viable (such as cases where we have a large training set and/or large set of environment features), LfODBN is a good alternative. However, in cases where CBR is viable, it is recommended to use TB as it outperformed DBNs at imitating state-based experts.

\subsubsection{Probabilistic Finite Automata}

A Probabilistic Finite Automata (PFA) in the context of imitation learning is defined as a 5 -tuple Automaton $=(A, S, \phi, \iota, \gamma)$, where $A$ is a finite set of actions, $S$ is a finite set of environment states, $\phi: S \times A \times S \rightarrow[0,1]$ is a function mapping the probability

of transitioning to environment state $s^{\prime} \in S$ from environment state $s \in S$ given an 
action $a \in A, \iota: S \rightarrow[0,1]$ is a function mapping each environment state to the probability of being an initial state, and $\gamma: S \rightarrow[0,1]$ is a function mapping each environment state to its probability of being a final state [3].

The authors of [3] estimate the functions $\phi$ and $\iota$ of the automaton using traces of the expert and Laplacian smoothing, to learn its behavior. This method requires the environment to be discrete in order to compute the approximations of the functions. This method also exclude the final state probability function $\gamma$, as it is not required to learn an expert's behavior. The resulting PFA is then used to stochastically select the action to perform instead of deterministically selecting the action that has the highest probability. In the next paragraphs, we detail how the functions of a PFA are used to determine the probability of each action given the trajectory of the agent.

Using the definition of a PFA, we can define the probability of a non-terminating trajectory of an agent $L$ using equation (3.1); where $\alpha=\left(a_{1}, \ldots a_{m}\right) \in A^{*}$ is a non terminating action trajectory, and $\sigma=\left(s_{1}, \ldots, s_{m}\right) \in S^{*}$ is a non terminating environment state trajectory.

$$
P_{L}(\alpha, \sigma)=\iota\left(s_{1}\right) \prod_{i=1}^{m-1} \phi\left(s_{i}, a_{i}, s_{i+1}\right)
$$

In the study of Tîrnăucă et al., they estimate the functions $\iota(s)$ and $\phi\left(s, a, s^{\prime}\right)$ in equation (3.1) using the trajectories of the expert and laplacian smoothing [3]. However, the environment needs to be discrete in order to compute the approximations of the functions. Hence, this method is not suitable in continuous environments where the set of possible environment states is not finite.

This technique was compared to decision trees [32], probabilistic neural networks [33], k-Nearest Neighbors (kNN), the RProp algorithm for multi-layer feedforward networks [34], and a Naive Bayes classifier in a discrete domain. Specifically, 
the authors use the vacuum cleaner domain (without dirt) to evaluate each imitation learning agent at imitating experts that exhibit reactive behavior and state-based behavior. They also evaluated how well PFAs imitate experts that have a stochastic component for which action they select. Their results indicated that the PFA learner outperformed all the techniques they tested at imitating all of the experts, except for a deterministic expert that uses information from its previous action and current environment state to select the next action (the CRASH expert from Section 3.2.2.3).

We can conclude that this method is suited to imitate experts that are not using the previous action to select their next action. Furthermore, we concluded that this method can not be used in continuous domains where the set of environment states or actions is infinite; since estimating the functions $\phi$ and $\iota$ involves counting the occurrences of each unique environment state and action in the trajectories of the expert. This technique is also not ideal in cases where the actions are deterministically selected by the expert, as it stochastically selects the action. Therefore, we need to use other models when an expert reasons on the actions it selects, rather than stochastically selecting it. However, this technique can be adapted to those situations by deterministically selecting the action with the highest probability rather than stochastically selecting the action.

\subsubsection{Case-Based Reasoning}

Traditional applications of Case-Based Reasoning (CBR) to imitation learning consider

each environment state and action pair from the expert's trajectory as a separate case in its training set, where future environment states are compared to the cases in this set [6]. Thus, the action taken in the case closest to the current environment state of the agent is selected by the agent. However, this method of comparing cases 
is not sufficient to imitate state-based behavior since it only considers the current environment state. Recall from Chapter 2, that reactive behavior is behavior that only considers the current state of the environment. Whereas, state-based behavior is behavior that considers the current state of the environment as well as information from past environment state(s) or action(s) previously executed by the agent. This motivated Gunaratne et al. [2] to introduce 5 new CBR similarity metrics that consider past environment states and actions when comparing cases to imitate state-based behavior:

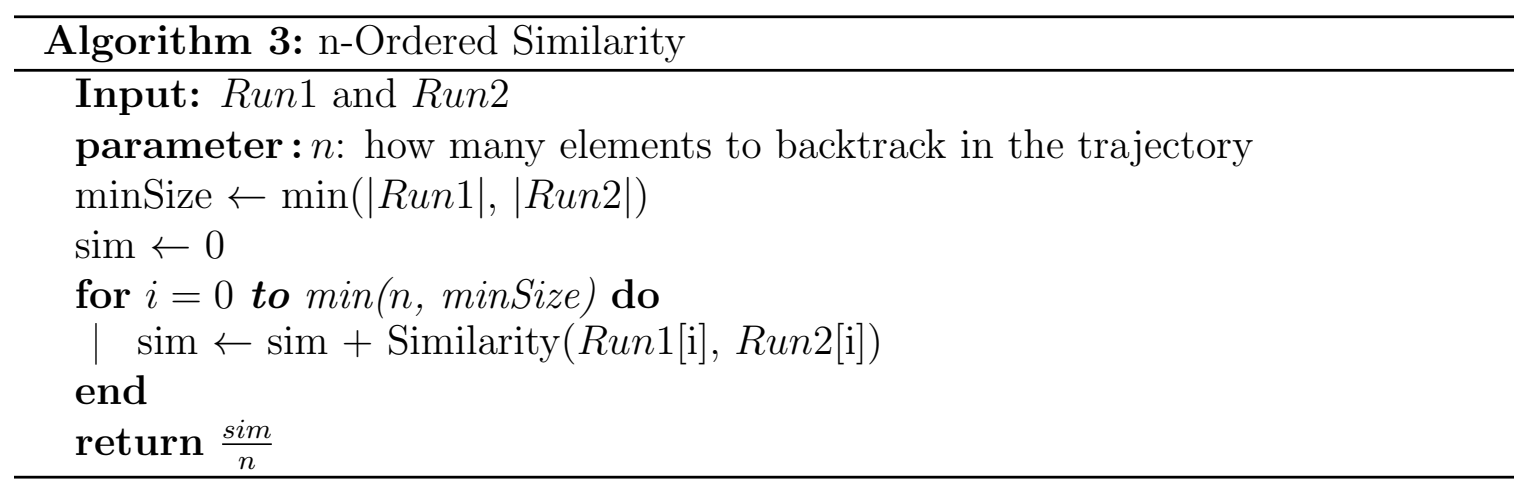

n-Ordered Similarity The n-ordered similarity metric compares the $n$ most recent elements of the trajectories as described in Algorithm 3, where the index $i=0$ of a run is the most recent state of the environment. The elements alternate between environment states and actions as the $n$ most recent elements of the trajectories are being compared.

Ordered Similarity The ordered similarity metric is the same algorithm as the n-ordered similarity; however, it compares the entire trajectory of the agent to the trajectories in its training set instead of using the hyper-parameter $n$. 


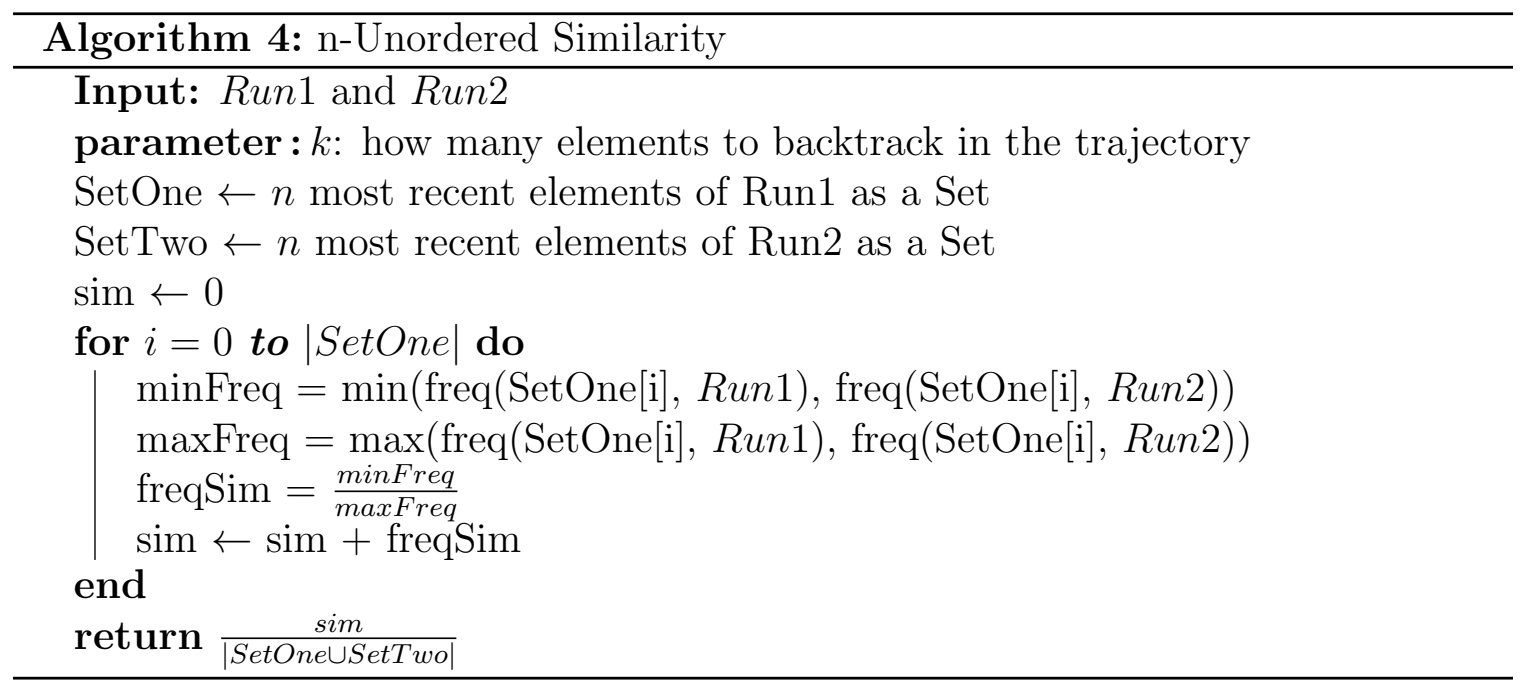

n-Unordered Similarity The n-unordered similarity metric computes the difference in the environment state and action frequencies between the two trajectories in the $n$ most recent elements of the trajectories (see Algorithm 4). This implies that it can only be used in domains where the set of environment states is finite (i.e. discrete environments).

Unordered Similarity The unordered similarity metric is an extension of the nunordered similarity metric that uses the entire trajectory of the agent rather than a preset hyper-parameter $n$.

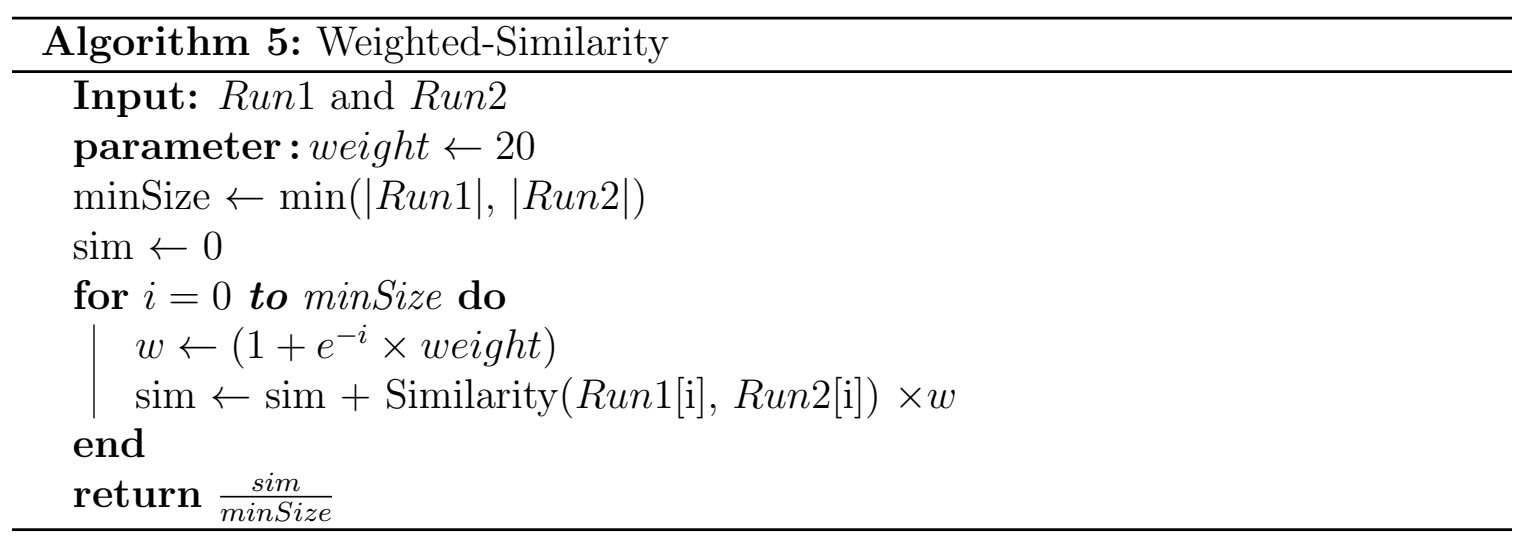


Weighted Similarity The weighted similarity metric computes the difference between two trajectories in the same way the ordered similarity metric computes it; however, it weighs the first 10 elements in an exponentially decreasing manner, whereas the elements in the rest of the trajectory are assigned a weight of 1 . We describe the weighted similarity metric in Algorithm 5.

It is apparent that all the similarity metrics proposed by Gunaratne et al. [2] make an assumption on the function $F: \mathcal{R}^{A} \rightarrow I$ that maps the set of runs that end with an action $\mathcal{R}^{A}$ to the set of hidden internal states of the expert $I$, similar to TB in Section 3.3.2. For example, the n-ordered similarity makes two assumptions on the expert: (1) the $\mathrm{n}$ most recent environment states and actions influence the hidden internal state of the expert and (2) the features of the environment states and actions are what influences the hidden internal state of the expert. Likewise, the n-unordered similarity makes the assumption that the frequency of each unique environment state and action is what influences the hidden internal state of the expert in addition to assumption (1) that n-ordered similarity makes.

The authors compared their proposed similarity metrics to each other and to TB. They used the same discrete vacuum cleaner domain (with dirt) used in the work of Ontañón et al. [1] to evaluate the similarity metrics they introduced. In addition to the Frequency expert they designed (refer to Section 3.2.2.2), they used the Fixed Sequence and ZigZag experts described in Section 3.2.2.2 that were designed by Ontañón et al. [1] to evaluate their metrics against TB. Their results were able to show that there was no similarity metric that is universally better than all other similarity metrics. Moreover, they found that TB under-performed relative to their similarity metrics when imitating experts that decide which action to take based on the frequency of past environment states (Frequency expert) and experts that repeat the same sequence 
of actions regardless of the environment state (Fixed Sequence expert). However, TB outperformed the similarity metrics they proposed when imitating behavior that required using information from previous environment states and the previous actions they have taken.

Thus, the conclusion of their study is that there is no CBR similarity metric that is universally better to use for imitating state-based behavior in the context of CBR. This motivates the need to propose techniques that do not rely on similarity metrics, in order to make no assumption on the function $F$. Ideally, a technique needs to be capable of learning the function $F$ for it to universally fit all kinds of state-based experts. Furthermore, we need the technique to be applicable to both continuous and discrete domains, as some CBR similarity metrics are constrained to discrete domains (such as the n-unordered and the unordered similarity).

\subsubsection{Conclusion}

We have discussed the techniques used to learn state-based behavior, and the situations that can make a technique preferable to other techniques in this section. We also discussed the limitations of each technique and highlighted how PFA, n-unordered and unordered similarity are limited to discrete domains. Moreover, we reviewed studies that indicate TB to be the best technique to use for most state-based behavior, except in cases where the behavior of the state-based expert does not follow the assumptions TB makes. Thus, we need to propose imitation learning techniques that make no assumption on the state-based expert's behavior. Moreover, DBN models such as LfODBN may be preferable to CBR and TB in situations where we lack the space requirements of a CBR technique and where the assumptions of $\mathrm{CBR}$ and $\mathrm{TB}$ are not met by the expert. In the next section, we will discuss the different metrics and 
testing methodologies utilized by the studies we discussed in this section to obtain results.

\subsection{Testing Methodologies and Metrics}

\subsubsection{Introduction}

Most of the studies we discussed in this chapter use prerecorded traces of experts to

obtain their results $[1,3,4,2]$. However, some of the results obtained by Tîrnăucă et al. [3] do not make use of prerecorded traces of experts. Instead, they compare a full trajectory of the agent to a full trajectory of the expert. In this section, we will discuss the two methods used to test imitation learning agents, the common metrics used in conjunction with each method, and the advantages and disadvantages of each testing method. The first class of testing methods we will discuss is offline testing: where the trained agent uses a prerecorded trace of the expert to select an action at each environment state. We call this type of testing "offline" as it does not include executing the agent in the environment. The second class of testing methods we will discuss is online testing: where the trained agent is executed in its environment, and its trajectory is compared to that of the expert.

\subsubsection{Offline Testing}

Offline testing involves running a trained imitation learning agent over a prerecorded trajectory obtained from the expert. Hence, the agent does not run in the environment; instead, it classifies each sub-trajectory that ends with an environment state at each 
time step of the expert's prerecorded trajectory. The metrics are then obtained by comparing the action the learning agent selected with the action in the prerecorded trajectory. The predictive accuracy and the F1-score are commonly used when performing offline testing $[20,1,3,4,2]$.

The predictive accuracy is defined in Equation (3.2), where $\#(L, T, i)=1$ if the agent $L$ predicts the same action as the expert in the expert's trajectory $T$ at time $i$, otherwise $\#(L, T, i)=0$.

$$
\operatorname{Acc}_{L}(T)=\frac{1}{m} \sum_{i=1}^{m} \#(L, T, i)
$$

The F1-score is the harmonic mean of the precision and recall of each action (i.e. class) defined in Equation (3.3).

$$
F_{1}=\frac{\text { precision } \cdot \text { recall }}{\text { precision }+ \text { recall }}
$$

This type of testing does not replicate what happens when the agent is executed in the environment. When an agent is executed in an environment, the next state of the environment is usually dependent on its previous state and the action of the agent. Hence, whenever the learning agent takes an action that is different from the expert, its next environment state will likely be different to that of the expert. Moreover, its next action may be different from the expert's action in the next time step as it is in a completely different environment state. As a result, all the studies that investigate the qualitative performance of their agents at imitating an expert, reported that their agents had high F1-scores and accuracies but fail to imitate the expert adequately when they run in the environment $[1,3,4]$. On the other hand, an advantage of using offline testing is that it is straight forward to obtain error measures by applying $\mathrm{k}$-folds 
cross validation or other traditional techniques. In the next section, we will discuss online testing that addresses the issues of offline testing.

\subsubsection{Online Testing}

Online testing involves executing the trained imitation agents in the environment without influencing its trajectory. Hence, the agent experiences the same environment states and trajectories that it would experience when deployed. A few studies make use of this testing method such as $[22,21,3]$. Some of those studies use domain specific metrics that evaluate how well the agent performs in its environment as in [22], rather than how well it imitates the expert. Other studies use metrics that were designed for their domain to compare the trajectory of the agent with the trajectory of the expert, as in the helicopter domain in [22]. In this section, we will only focus on cross-domain metrics that evaluate how well the agent imitates the expert.

Currently, the only cross-domain metric used in online testing techniques to measure the distance between the expert's trajectory and the agent's trajectory is the Monte Carlo distance proposed by Tîrnăucă et al. [3] The Monte Carlo distance is the normalized log of the count of state-action pairs that are identical in trajectories $T$ and $T^{\prime}$ defined in Equation (3.4).

$$
H\left(T, T^{\prime}\right)=-\frac{1}{n} \sum_{i=1}^{n} \log \left[\frac{\sum_{j=1}^{m} \Pi_{\left\{o_{j}^{\prime}\right\}}\left(o_{i}\right)+1}{m+|S| \times|A|}\right]
$$

Where $o_{i}=\left(s_{i}, a_{i}\right)$ is an observation and action pair in the trained agent's trace $T, n$ is the length of the agent's trace, $o_{j}^{\prime}=\left(s_{j}, a_{j}\right)$ is an observation and action pair in the expert's trace $T^{\prime}, m$ is the length of the expert's trace, and $\Pi_{\left\{o_{j}^{\prime}\right\}}$ is the indicator function of set $\left\{o_{j}^{\prime}\right\}$. This metric measures the distance between the $P(A \mid S)$ 
distribution of the expert's trajectory and the agent's trajectory; where $A$ is the set of actions and $S$ is the set of environment states.

While this metric provides a good estimate of how well the agent is imitating the expert, it is only evaluating the reactive component of the trajectories of each agent. Ideally, we would like to compare $P\left(A \mid R^{S}\right)$ in cases where the expert is state-based;

where $R^{S}$ is the set of runs that end with an environment state. However, since $R^{S}$ is not finite or at least very large in most domains, $P\left(A \mid R^{S}\right)$ is sparse and is impractical to sample. This makes $P\left(A \mid R^{S}\right)$ not a very useful distribution when comparing an agent to an expert.

Despite this, the Monte Carlo distance metric still correlates with the qualitative assessment of an imitation learner. Imitation learners that qualitatively imitate an expert better than other learners always had a lower Monte Carlo distance in the research of Tîrnăucă et al. [3]. Hence, it still provides a much better alternative to the metrics used in offline testing. On the other hand, it is not easy to retrieve the error of the Monte Carlo distance metric; since validation techniques such as k-fold cross validation can not be applied when performing online testing.

\subsubsection{Conclusion}

While offline testing techniques are simple to apply and calculate, they are not ideal when evaluating state-based imitation learning agents. This is because the environment state distribution the learner visits along its trajectory in practice is different to what the expert visits. Hence, the correct way of testing state-based imitation learners is using online testing since the agents are tested on their own trajectories. We also saw how studies obtained high accuracy and F1-scores when using offline testing, while reporting that their agents do not imitate the experts adequately when deployed in 
the environment $[1,3,4]$.

\subsection{Conclusion}

In this chapter, we discussed some of the benchmarks used to evaluate imitation learning techniques in the field of imitation learning. It was clear that there is a lack of testing state-based imitation learning in complex continuous domains, such as RoboCup, without discretizing its environment. We also studied the different techniques used in imitation learning, where we concluded that TB is the ideal technique in most scenarios unless the expert violates the two assumptions of TB: (1) the hidden internal state of the expert is dependent on the most recent events and (2) it is dependent on a certain feature(s) of a past environment state or action. It is also apparent that neural networks which are capable of learning state-based behavior, are capable of learning the function $F: \mathcal{R}^{A} \rightarrow I$ (refer to Section 3.3.2), without making an assumption on the expert; yet, they were never compared to any of the techniques we mentioned in this chapter. Lastly, we discussed the testing methodologies and metrics used to evaluate imitation learning agents. Where we learnt about the inadequacies of results that are obtained from offline testing, and how online testing provides more reliable results that correlate with the qualitative assessment of imitation learning agents.

In the next chapter, we will remodel the RoboCup soccer environment state vector to preserve its continuous and complex nature. Additionally, we will design a wider variety of state-based RoboCup soccer experts that rely on different features of the run (i.e. have different functions $F$ ). In return, this will provide us with a more realistic benchmark to evaluate imitation learning techniques. We will also discuss 
the methodologies used to train, test, and evaluate state-based imitation learning techniques used in this thesis. Furthermore, we will introduce metrics for evaluating state-based imitation learners that make use of online testing in this thesis. We will also describe an online testing method of deriving the F1-score and accuracy; which provides results that correlate with the qualitative performance of the agents. Lastly, we will address the issue of techniques that make assumptions on the function $F$, by proposing the use of LSTM networks with the assistance of DAgger to imitate state-based behavior. 


\section{Chapter 4}

\section{Methodology}

\subsection{Introduction}

In this chapter, we start by discussing the benchmarks and experts we will use to test our imitation learning techniques in Section 4.2. In addition, we remodel the environment state features and action space of our RoboCup soccer agents, to test the techniques we will evaluate in this thesis using continuous features. Next, we will discuss the imitation learning techniques we will use in our experiments in Section 4.3, with emphasis on the technique we are proposing: Long Short-Term Memory (LSTM) networks. This technique should enable us to learn all types of state-based behavior without making an assumption on the expert. Moreover, we describe the architecture of the neural networks we used and the training methodology of each imitation learning technique. Lastly, we discuss the metrics and testing methodology used in our experiments in Section 4.4, where we concentrate on the metrics we propose in the context of online testing. Additionally, we will describe an online testing method of obtaining F1-scores that correlate with the qualitative performance of the agents. 


\subsection{Benchmarks}

To evaluate our proposed imitation learning techniques and compare them to previous techniques, we used two domains: (1) the discrete vacuum cleaner with dirt domain from Section 3.2.2.2, and (2) the continuous RoboCup soccer model. For the vacuum cleaner domain, we used the Smart Random, Smart Straight Line, Fixed Sequence, and ZigZag experts from Section 3.2.2.2.

Past work that made use of RoboCup soccer [4] chose to discretize the input that is received from the RoboCup simulator which leads to the possible loss of information, and accuracy. This transformation will not be needed for the techniques we use. In section Section 4.2.1, we will show how we represent the input to our agents for the RoboCup domain and the actions the agents can take. Next, we will define new state-based experts for the RoboCup soccer domain which feature a variety of different behaviors in Section 4.2.2, where we aim to provide experts that test the ability of imitation learning techniques at capturing different types of state-based behavior in a continuous domain. We used our model of the RoboCup soccer domain and the experts we designed to evaluate the imitation learning techniques used in our experiments.

\subsubsection{Representing the Continuous Input from the RoboCup Soccer Environment}

In this section, we remodel the environment state features and action space of the RoboCup soccer agents to preserve the continuous nature of the domain. This will allow us to test the limits of state-based imitation learning techniques that are suitable for use in a continuous environment. Additionally, this model of RoboCup will be used 
to compare those techniques to the LSTM technique we will propose in Section 4.3.

This section is divided into two parts: Section 4.2.1.1 where we define the environment state of the agent in RoboCup soccer, and Section 4.2.1.2 where we define the action space of the agent.

\subsubsection{RoboCup Soccer Environment Space}

As we mentioned in Chapter 3, RoboCup soccer is a virtual soccer simulation

\begin{tabular}{|c|c|}
\hline Feature & Description \\
\hline Ball direction & $\begin{array}{l}\text { The angle of the ball relative to the } \\
\text { agent. }\end{array}$ \\
\hline Ball distance & The distance of the ball from the agent. \\
\hline Goal direction & $\begin{array}{l}\text { The angle of the enemy's goal from the } \\
\text { agent. }\end{array}$ \\
\hline Goal distance & The distance of the goal from the agent. \\
\hline Self goal direction & $\begin{array}{l}\text { The angle of the agent's goal from the } \\
\text { agent. }\end{array}$ \\
\hline Self goal distance & $\begin{array}{l}\text { The distance of the agent's goal from } \\
\text { the agent. }\end{array}$ \\
\hline Is ball visible? & $\begin{array}{l}\text { A boolean: } 1 \text { if the ball is in the field of } \\
\text { view of the agent, otherwise } 0 .\end{array}$ \\
\hline Is goal visible? & $\begin{array}{l}\text { A boolean: } 1 \text { if the enemy's goal is in } \\
\text { the field of view of the agent, otherwise } \\
0 \text {. }\end{array}$ \\
\hline Is self goal visible? & $\begin{array}{l}\text { A boolean: } 1 \text { if the agent's goal is in the } \\
\text { field of view of the agent, otherwise } 0 \text {. }\end{array}$ \\
\hline
\end{tabular}

Table 4.1: Description of the environment vector features

environment that provides each agent with details about its environment in a string format. The environment consists of the ball in the soccer field, the two goals, and the other agents in the field (enemy team and friendly team). The agent receives a string 
that contains the name of each object in its field of view including its angle from the agent and its direction. The agents do not have full information about the environment as they are limited by their field of view, and as such the environment is partially observable. This poses a challenge in creating a vector that models the perception of an agent, as some information may not be available. Moreover, the RoboCup soccer environment is dynamic and non-deterministic; hence, the environment state of the agent is also influenced by factors outside the scope of the agent, such as other players in the field, and the agent's next environment state is not deterministically determined by its action and the environment state it took the action upon.

In order to deal with objects such as the ball and other players that may not be in the field of vision of the agent, the environment vector contains a binary field stating if an object is visible or invisible and 2 fields containing the direction and distance information for every possible object in the simulation. When objects are no longer in the field of view of the agent, their visibility field is set to 0 and the direction and distance information from the previous vector are used. We assume that all our experts take their decisions based on the visibility, distance and direction of the ball and the two goals. Hence, we are omitting information about other agents in the field. We model the environment with a vector that consists of 9 features: the distance, direction and visibility of the ball, the enemy goal and the agent's own goal. A detailed description of the features encoded in the environment state vector used in our approach is presented in Table 4.1 .

\subsubsection{Action Space}

The action space of the agent is discretized to contain only the actions that are performed by the state-based experts the agent is trying to imitate. We summarize 
the available actions for an agent to take in Table 4.2. The action parameters are pre-defined; yet, the agent still has to select the correct action.

\begin{tabular}{ll}
\hline Action & Description \\
\hline Turn positive & Turn 40 degrees. \\
Turn to ball & Turn to ball direction (ball direction \\
& retrieved from feature vector). \\
Dash & Dash forward with power 10. \\
& Kick with power 100 towards the goal \\
Kick to goal & direction (goal direction retrieved from \\
& feature vector). \\
Turn negative & Turn -40 degrees. \\
\hline
\end{tabular}

Table 4.2: The action space of the agent

\subsubsection{Conclusion}

We remodelled the environment state input of RoboCup soccer into a vector that preserves the continuous nature of the domain. This environment state vector provides information on the visibility of relevant objects (the ball and the two goals in this case) and information about the distance and direction of each object in the field of view of the agent. We also defined the 5 actions a RoboCup soccer agent can perform: dash, turn to the right, turn to the left, turn to the ball, and kick to the goal. In the next section, we will define some state-based experts that use the RoboCup soccer feature vector we defined and a hidden internal state to select an action. 


\subsubsection{RoboCup Soccer Experts}

\subsubsection{Introduction}

To evaluate the performance of each method, we need to categorize different criteria of state-based behavior and test the ability of each approach to capture the defined criteria. We attempted to design a variety of programmed experts that match the experts we have in the vacuum cleaner domain. We do not make use of human experts as they are prone to error and the behavior of a human expert may change over time. Moreover, it takes much longer to produce demonstrations using human experts than programmed experts. We also aim to compare the behavior of each approach relative to the expert; therefore, using a programmed expert with a well-defined behavior gives us the ability to objectively compare the behavior of each technique with the expert's behavior.

We use a reactive expert to test the ability of each technique at capturing behavior that purely relies on the current state of the environment, and not the internal state of the agent. The rest of the experts are state-based experts that were designed to test the ability of each method to learn the following: transitioning from one hidden internal state to another based on the number of time steps, transitioning internal state based on an environment trigger, persisting short-term information, persisting long-term information, and retaining a large hidden internal state space. In total, we use one reactive expert and five state based experts that each test a dimension in the aforementioned criteria. We describe the behavior of each expert below and highlight the dimension(s) of state-based behavior they test.

Krislet Expert The Krislet Expert, designed by Langner [35], tests the ability of an imitation learning agent at capturing reactive behavior. As we recall from Sec- 
tion 3.2.3.2, it turns in the positive direction if the ball is not in the vision of the expert and if it is, it turns towards the ball. Once aligned with the ball, it dashes to the ball till the ball is in kicking range. If the goal is not in the vision of the expert when it is in kick range, it turns in the positive direction till it can see the goal. Once it sees the goal and the ball is in kicking range it kicks the ball to the goal. The decision tree of the Krislet expert is described in Figure 4.1.

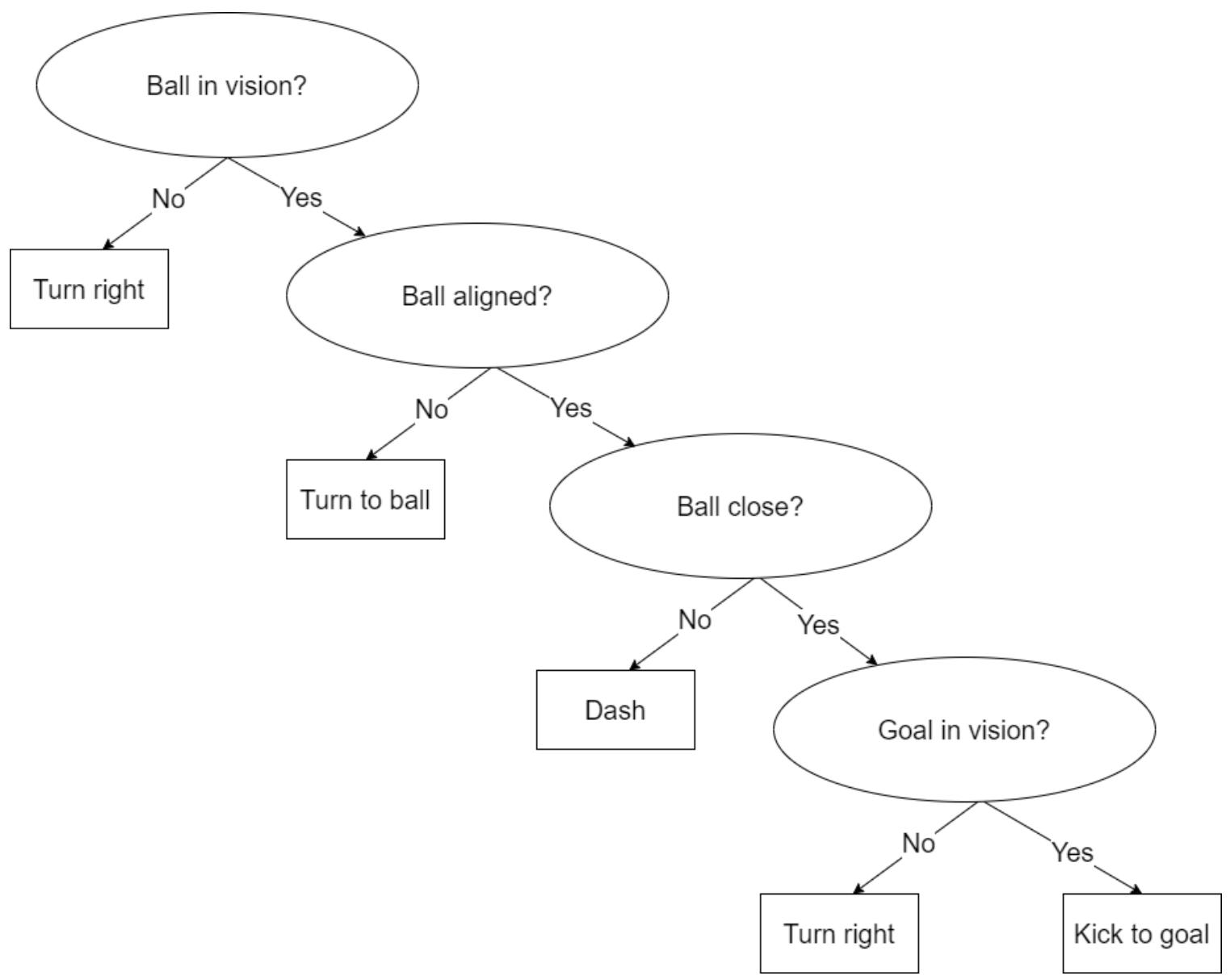

Figure 4.1: Krislet Expert Decision Tree

Switch Expert The Switch Expert provides a basic test of a model's the ability to capture state-based behavior. It follows the behavior of the Krislet Expert; however, 


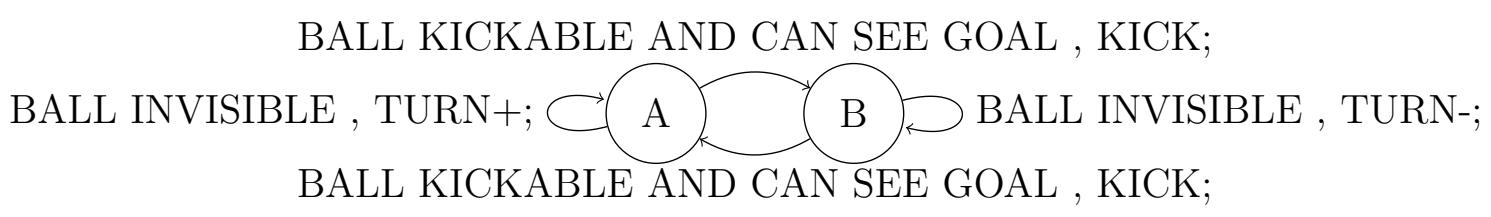

Figure 4.2: Switch Expert Automaton

whenever it kicks the ball to the goal it switches the direction it turns in when it can not see the ball (figure 4.2). It is the simplest state-based expert behavior we thought of as we can prove that it is state-based with a short trajectory: BALL INVISIBLE $\stackrel{T U R N+}{\longrightarrow}$ BALL CLOSE AND CAN SEE GOAL $\stackrel{K I C K}{\longrightarrow}$ BALL INVISIBLE $\stackrel{T U R N-}{\longrightarrow}$. BALL CLOSE AND CAN SEE GOAL, KICK, count=0; BALL IN VISION , TURN_BALL; $C$ C B B BLL IN VISION , DASH, count++;

Figure 4.3: Offense Expert Automaton

Offense Expert The Offense Expert tests the ability of a model to capture a state transition that is reliant on the number of environment states visited, as opposed to an environment or action trigger to cause the transition. Hence, its hidden internal state can not be directly deduced from the features of previous environment states or actions. Again, it uses the same logic as the Krislet Expert; however, whenever it kicks the ball, it continuously dashes to the ball if it sees the ball, whether or not the agent is aligned with the ball for 20 environment states (see Figure 4.3).

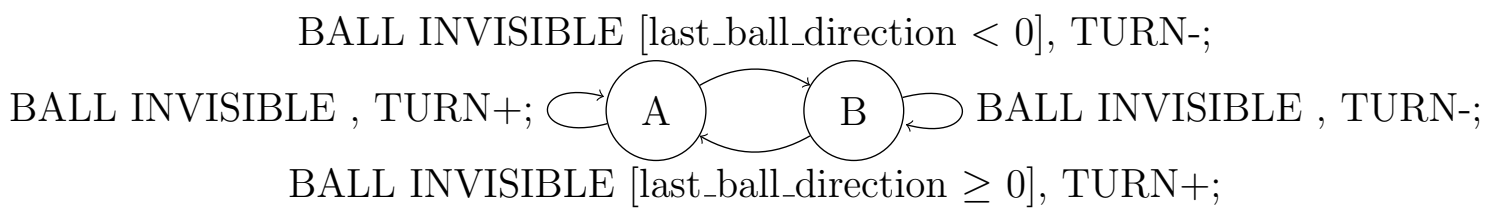

Figure 4.4: Pro Expert Automaton 
Pro Expert This Expert tests the ability of a model to capture transitions that rely on a feature of a particular environment state in the short-term past. It turns towards the direction it last saw the ball when it no longer sees the ball; otherwise, it is identical to the Krislet Expert (refer to Figure 4.4). Hence, the expert may or may not transition its state when it loses sight of the ball. This allows us to test the short-term memory of the model and its ability to identify the relevant feature in its memory that decides a state transition. On average, this expert uses information that is not older than 4.3 environment states in the past with a 1.8 standard deviation (collected from a full match of the expert).

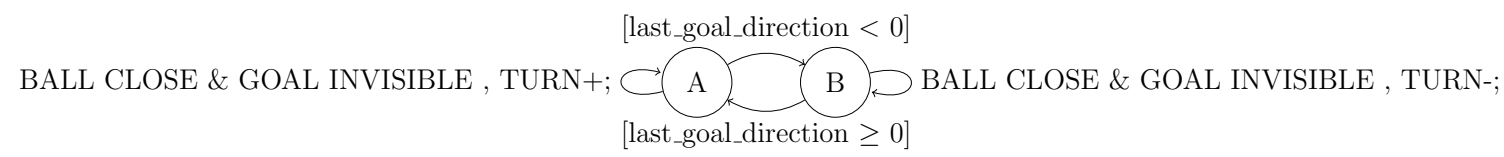

Figure 4.5: Goal Expert Automaton

Goal Expert The Goal Expert tests the ability of a model to remember information that was far in the past. It turns towards the direction it last saw the goal when it is trying to find the goal; otherwise, it is identical to the Krislet Expert (see Figure 4.5). As opposed to the Pro Expert, the information needed to take a decision on which direction to turn is much longer in the past. This is because if the expert does not see the goal, it implies that the ball is in the possession of the enemy team and the expert is chasing the ball back towards its own goal. Hence, it takes a long time for it to reach the ball before it turns looking for the enemy's goal. This tests the long-term memory of the model. The average number of environment states this expert visits before it needs to recall the position of the goal is 193 environment states with a standard deviation of 117 environment states (retrieved from a soccer match of the expert). 
Pro Goal Expert This Expert tests both the long-term and short-term memory of the model simultaneously. It memorizes both the last direction it saw the ball in and the last direction it saw the goal in, combining the logic of the Goal Expert and the Pro Expert. This expert has 4 states as opposed to the previous state-based experts. This expert also tests the ability of a model to capture a larger hidden internal state space.

\subsubsection{Conclusion}

We use the Krislet expert to test the basic ability of capturing reactive behavior and propose the Switch expert to test the ability of capturing state-based behavior in the RoboCup soccer domain. The rest of the experts we proposed test a technique's capability at learning different dimensions of state-based behavior: the offense expert tests the ability of a method to recognize internal state transitions that rely on time rather than the current environment state, the pro expert tests the ability of a method to remember environment state features that were available in the short-term past, the goal expert tests the ability of a technique to remember environment state features that were available in the long-term past, and the pro goal expert tests the former two dimensions in addition to a larger internal state-space. This provides us with a richer variety of benchmarks in the RoboCup soccer domain to test state-based imitation learning techniques.

\subsubsection{Conclusion}

In this section, we discussed the domains and experts we will use in our experiments. We also provided new experts for the RoboCup soccer domain that test a variety of state-based behavior and a new model of the RoboCup soccer domain that maintains 
the continuous nature of the domain. In addition to the benchmarks provided by the vaccuum cleaner domain, those contributions will provide realistic benchmarks where we can test the imitation learning techniques we use in this thesis.

\subsection{Techniques}

Before we present the neural networks that we propose for imitating the RoboCup soccer and vacuum cleaner experts, we will list the state-of-the-art techniques that we will use in our experiments for each domain, their training methodology and hyperparameters. Recollect from Section 3.3.5 that the ordered and weighted similarity metrics are extensions of the n-ordered similarity metric that uses the entire trajectory of the agent instead of the last $n$ elements of its trajectory. Thus, we decided to not use the ordered and weighted similarity metrics in Case-Based Reasoning (CBR), as we will show in the next chapter that the n-ordered similarity metric extremely under-performs when subjected to experts that require recalling a large number of past events (i.e. experts that require a large hyper-parameter $n$ ).

In the RoboCup soccer domain, we use Temporal Backtracking (TB) and CBR with the n-ordered similarity metric to compare them to the techniques we proposed in Section 4.3.1. The training set of $\mathrm{TB}$ and $\mathrm{CBR}$ with the n-ordered similarity metric are obtained using Dataset Aggregation (DAgger) (refer to Chapter 3), where each case the learner visits during its execution in the environment is added to its training set with the action the expert would have taken. In the case of CBR, we always set $k=5$ to select the 5 nearest neighbors. We also compute the similarity of an

environment state using the average euclidean distance which was used in the work of Gunaratne et al. [4]: 


$$
\operatorname{sim}\left(s_{1}, s_{2}\right)=\sum_{i=0}^{m} \frac{1}{1+, s_{1}[i]-s_{2}[i]}
$$

Where $s_{1}$ and $s_{2}$ are the environment states, $s_{n}[i]$ is feature $i$ of the environment state, and $m$ is the number of features in the largest environment state vector. When

a feature is missing from one of the environment states, we replace $\frac{1}{1+, s_{1}[i]-s_{2}[i]}$, with a penalty of -10 . Hence, we do not include the visibility features in the environment state vector of CBR and TB techniques. Similarly, if two actions are the same their similarity is 1 , otherwise, their similarity is 0 .

In the context of the vacuum cleaner domain, we use LfODBN, in addition to TB, CBR $(k=5)$ with the n-ordered similarity metric, and our proposed techniques. However, since the vacuum cleaner domain is tested by introducing the agent in a new map, we do not employ DAgger to collect the training set of any of the techniques we use. This is because changing the map of the agent changes the environment state distribution of the agent, and the objective of DAgger is to minimize the difference between the environment state distribution of the training set and the environment state distribution of the agent when it is deployed. In the next subsection, we will discuss the techniques we propose to use in the RoboCup soccer and vacuum cleaner domains.

\subsubsection{Proposed Techniques}

Recall from Chapter 3, CBR and TB are two techniques that make assumptions on the function $F: \mathcal{R}^{A} \rightarrow I$, which maps the runs that end with an action to a hidden internal state in the context of state-based experts. It is essential for a technique to learn the function $F$ in order to adequately imitate state-based experts. However, 
CBR sets a fixed function $F$ when we select a similarity metric. Similarly, TB makes an assumption that the function $F$ has a recency bias.

In this section, we propose the use of Long Short-Term Memory (LSTM) neural networks which are capable of learning the function $F$. This makes them a contender to be a technique that is universally applicable to all types of state-based behavior. As a baseline to compare the LSTM approach, we will start by describing the architecture and training technique of a simple fully connected Multi-Layer Perceptron (MLP).

\subsubsection{MLP}

The MLP neural network consists of 2 fully connected hidden layers of size 500 each, with the ReLU activation function applied after each hidden layer. We also apply batch normalization on the input vector and on the output of each hidden layer to improve the convergence of the network's parameters. Softmax is applied on the final output of the network to select which action to take. The neural network layers are defined by the configuration 9-500-500-5 in the case of the RoboCup soccer domain and 8-500-500-5 in the case of the vacuum cleaner domain. This is because the environment state vector of the RoboCup soccer domain contains 9 features; whereas, the environment state vector of the vacuum cleaner domain consists of 8 features.

The MLP model, $\hat{\pi}$, was trained using DAgger presented in Algorithm 1 in the RoboCup soccer domain. At every step of a run, the imitation learning agent selects an action (using the neural network) based on the current perception of the environment and performs the action they selected. Next, we query the expert, $\pi^{*}$, for the action they would have taken at that step of the run and add the environment state and action pair to the training set of the agent. The agent runs 10 epochs of training on the new dataset at the end of each 10-step trajectory using the cross-entropy loss 
criterion and adaptive learning rate optimization (Adam). We set the hyper-parameter

$\beta_{i}=0$ to train the imitation learning agent $\hat{A g} g_{i+1}$ on the dataset collected purely from the environment state distribution of previous $\hat{A g} g_{i}$. We also return the last $\hat{A g} g_{i}$ instead of returning the best performing $\hat{A g} g_{i}$.

In the case of the vacuum cleaner domain, we train the MLP for 1000 epochs over its training set that is obtained from demonstrations of the expert in 6 vacuum cleaner maps. Since the purpose of DAgger is to train the model over its own environment state distribution and the vacuum cleaner agent is meant to be deployed in a new vacuum cleaner map (i.e. a completely new environment state distribution), we do not need to apply DAgger.

\subsubsection{LSTM}

The LSTM neural network we propose consists of a single LSTM cell that has a hidden dimension of 128 (i.e. $h_{t}$ from Equation (2.17) belongs to the vector space $\mathbb{R}^{128}$ ). The ReLU activation function is applied to the output of the LSTM cell, followed by a fully connected layer with the configuration 128-5, and a softmax function that outputs the final action selected. Batch normalization is applied on the input vector as well as between the ReLU activation function and the fully connected layer as a form of regularization.

The LSTM model was trained using the same method and hyper-parameters as the MLP in the case of the RoboCup soccer domain. Refer to Section 4.3.1.1, for more details on the training method and hyper-parameters.

Since the run of the agent is a sequence, the LSTM's hidden state is reset after training on the training sequence (i.e. the entire dataset) and at the start of every new run. 
In the vacuum cleaner domain, we train the LSTM model for 1000 epochs over its training set instead of using DAgger, similar to the MLP model in Section 4.3.1.1.

\subsection{Metrics and Testing Methodology}

In Chapter 3, we discussed the different testing methodologies and metrics adopted in previous studies. In addition, we highlighted the issues that offline testing introduces when obtaining its metrics. While offline testing allows us to easily calculate the errors of our results, its results do not reflect the qualitative performance of the imitation learning techniques. Thus, we will employ online testing, to obtain all our results, including the F1-scores.

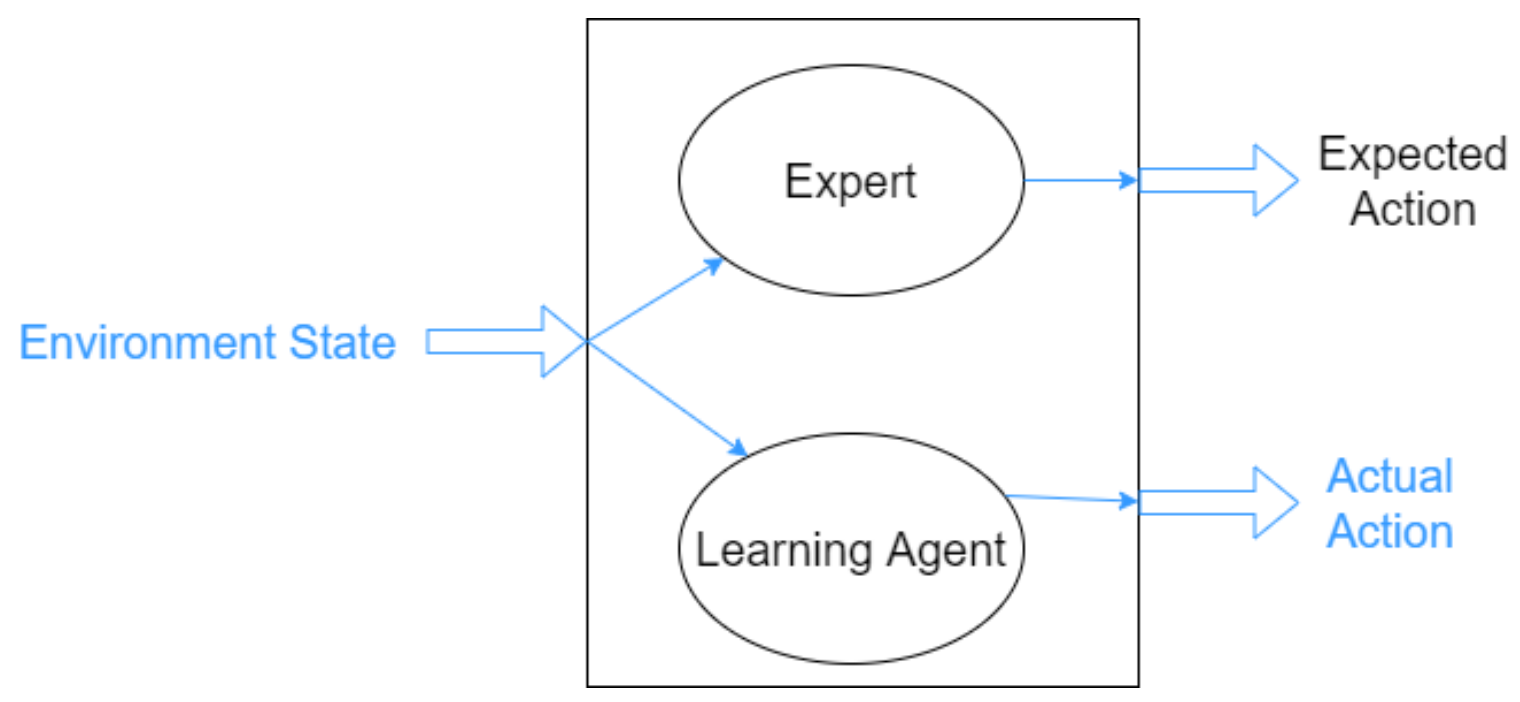

Figure 4.6: The online testing methodology of obtaining the F1 score. The blue action is the action performed in the environment, and the expected action is what is used to calculate the F1 score.

In order to obtain the F1-scores in our experiments, we will execute the imitation learning agents in their respective domain while querying the expert on the action they should have taken given their current trajectory (refer to Figure 4.6). This method 
of obtaining the F1-score should correlate with the qualitative performance of the trained agent; as opposed to the F1-score obtained from using the trained agent to predict the action of the expert on the expert's trajectory.

While most studies used the offline testing method of obtaining results, only one study in the vacuum cleaner domain proposed the use of a generic cross-domain online testing metric: the Monte Carlo distance [3]. In Section 4.4.1, we will propose cross-domain metrics for use in online testing to measure the distance between different distributions within the trajectory of the expert and the trained agent. We hypothesize that those metrics will provide us with more insight than the Monte Carlo distance on the behavior of the trained agent. In the next chapter, we will use those metrics and compare them to the Monte Carlo distance to (1) evaluate our techniques, and (2) learn about the insight they provide on the performance of imitation learning agents that other metrics do not provide. This work was also accepted and presented at The International FLAIRS Conference Proceedings, volume 34, Apr. 2021.

\subsubsection{Proposed Metrics}

Currently all the cross-domain performance metrics used in the field of imitation learning involve measuring the distance between the agent's and expert's $P(A, S)$. For example, the accuracy metric measures the percentage of time the agent selects the correct action at each environment state, and the Monte Carlo distance involves counting the number of identical state-action pairs in the agent's trajectory that are present in the expert's trajectory (assuming the model only takes the current environment state as input). However, recall from Chapter 2 that, unless the agent is reactive, an agent maps an entire trajectory ending with a state to an action. Hence, we can see that all the cross-domain metrics we currently use measure the reactive 
component of how well an agent imitates an expert. In order to test how well an agent imitates an expert that may be state-based, we need to use metrics that measure the performance of an agent over its $P\left(A, \mathcal{R}^{S}\right)$ distribution (the joint distribution of actions and its trajectory). Unfortunately, $\mathcal{R}^{S}$ is a very large set making it impossible to measure the agent's imitation performance over its $P\left(A, \mathcal{R}^{S}\right)$ distribution directly. Also, this probability distribution is likely to be very sparse and sensitive to small variations. However, we can measure it indirectly by using selective sub-sequences from a trajectory.

We propose using $P(S), P\left(S_{t}, S_{t-1}\right), P(A), P\left(A_{t}, A_{t-1}\right)$ and $P(A, S)$ as estimations of the trajectory of the agent and the expert. We can easily extract those distributions from the trajectory of the agent and the expert, and compare them to each other. The goal of the metrics we propose is to measure how well the agent generalized the behavior of the expert; hence, we did not use higher order distributions such as $P\left(S_{t}, S_{t-1}, S_{t-2}\right)$ and $P\left(A_{t}, A_{t-1}, A_{t-2}\right)$. Moreover, higher order distributions are likely to be sparse in comparison to lower order distributions. We use the $\chi^{2}$ statistic to measure the distance between the two distributions because, unlike the Monte Carlo distance, it is not computationally expensive to compute. In addition, it allows us to test the indistinguishability of the agent's distribution from the expert's distribution at a chosen confidence level. The $\chi^{2}$ statistic is calculated using the expected frequency $m_{i}$ of each possible value of the random variable in the distribution, its observed frequency $x_{i}$, and the number of possible values the random variable can take $k$, using Equation (4.2). We calculate the $\chi^{2}$ statistic under the null hypothesis that the agent's distribution and the expert's distribution are independent. In the following paragraphs, we provide the intuition behind the use of each distribution as an imitation learning metric and how each metric is calculated. 


$$
\chi^{2}=\sum_{i=1}^{k} \frac{\left(x_{i}-m_{i}\right)^{2}}{m_{i}}
$$

$\chi_{\mathbf{P}(\mathbf{S})}^{\mathbf{2}} \quad$ We compute this metric by recording the frequency of each unique environment state visited by the learning agent and the expert in their respective trajectories, then calculating the Chi-squared $\left(\chi^{2}\right)$ statistic using the frequency table constructed. Hence, this metric measures the difference between the distribution of states visited by the learning agent in its trajectory with that visited by the expert. Thus, this metric should be useful in comparing imitation learning agents, since an agent should be visiting the same environment state distribution of the expert if it is properly imitating the expert, regardless of the expert's type. Moreover, $\chi_{P(S)}^{2}$ does not consider the sequence of environment states the agent visits when comparing it to the expert; hence, it is oriented towards evaluating which techniques generalize rather than replicate the behavior of the expert better. However, it may not necessarily prove that the agent is imitating the expert correctly if there are multiple behaviors that can produce identical $P(S)$ distributions.

$\chi_{\mathbf{P}(\mathbf{A})}^{\mathbf{2}} \quad$ This metric compares the distribution of actions taken in the learning agent's trajectory to that of the expert's trajectory. As opposed to $\chi_{P(S)}^{2}$, this metric does not guarantee that two agents have the same behavior. This is because if an agent stochastically selects actions based on the action distribution of the expert without regard of its environment state, it will have the same $P(A)$ as the expert. However, it lets us know at a high level whether the agent is at least imitating the distribution of actions of the expert. This metric should be useful in cases where the expert takes actions stochastically, such as the Smart Random expert from Section 3.2.2.2. 
$\chi_{\mathbf{P}(\mathbf{A}, \mathbf{S})}^{\mathbf{2}}$ This metric compares the distribution of actions taken at each unique environment state of the agent with that of the expert. This metric is analogous to the accuracy and the F1 score; however, it measures deviation and as such, maximizing accuracy is the same as minimizing $\chi_{P(A, S)}^{2}$. It is also obvious that this metric is analogous to the concept of the Monte Carlo distance metric with a model that takes the current environment state as input. As the agent approaches perfect imitation of the expert, the $\chi_{P(A, S)}^{2}$ metric approaches 0. Again, it may not be ideal to measure how well an agent imitates an expert using this metric if the expert is state-based, since it may have an identical $P(A, S)$ to the expert but does not perform them based on the correct conditions that are required from past states.

$\chi_{\mathbf{P}\left(\mathbf{S}_{t}, \mathbf{S}_{t-1}\right)}^{\mathbf{2}} \quad$ This metric compares the joint distribution of unique environment states visited and the previous environment state of the agent's trajectory with the expert's trajectory. For this metric, we record the frequency of each unique state-to-state transition in the table used to compute the $\chi^{2}$ statistic. The goal of $\chi_{P\left(S_{t}, S_{t-1}\right)}^{2}$ is to measure how well the agent imitates the state transition distribution of the expert. As opposed to $P(S)$, this metric can prove that the behavior of the agent converged to the expert's behavior if the environment's state transitions depend on the action taken. This is because the agent needs to select the same actions as the expert at the correct states to match the expert's $P\left(S_{t}, S_{t-1}\right)$. Therefore, it can be thought of as a less generalized version of $\chi_{P(S)}^{2}$, since it requires the agent to not only visit the same distribution of environment states as the expert but also to maximize the chance of transitioning to the correct next state (which is achieved by selecting the correct action). 
$\chi_{\mathbf{P}\left(\mathbf{A}_{\mathbf{t}}, \mathbf{A}_{\mathbf{t}-1}\right)}^{\mathbf{2}} \quad$ This metric compares the joint distribution of actions and the previous action of the agent's trajectory with the expert's trajectory. $\chi_{P\left(A_{t}, A_{t-1}\right)}^{2}$ requires the agent to select a similar sequence of actions as the expert. This is useful in cases where an expert never performs a certain action after another and in cases where an expert frequently performs a distinct sequence of actions. Experts that exhibit those types of behavior are often state-based; thus, this metric is often useful to compare imitation learning agents when the expert is state-based.

To calculate the $\chi^{2}$ metrics and the Monte Carlo distance, we need a discrete environment space; however, the environment state space of RoboCup soccer is continuous. Therefore, we discretize the environment state space of RoboCup to only contain the set of mutually exclusive cases that the Krislet expert considers when taking an action to calculate $\chi^{2}$. Those cases contain no information about the internal state of the agent nor past environment states because the Krislet expert is a reactive expert. Equations (4.3) to (4.8) show an example calculation of the $\chi_{P(S)}^{2}$ statistic using the environment state data obtained from a RoboCup soccer experiment in Table 4.3.

\begin{tabular}{|c|c|c|c|c|c|c|}
\hline Model & $\begin{array}{l}\text { Ball } \\
\text { visible }\end{array}$ & $\begin{array}{l}\text { Ball } \\
\text { aligned }\end{array}$ & $\begin{array}{l}\text { Ball } \\
\text { kickable } \\
\text { and agent } \\
\text { can see } \\
\text { goal }\end{array}$ & $\begin{array}{l}\text { Ball not } \\
\text { in vision }\end{array}$ & $\begin{array}{l}\text { Ball } \\
\text { kickable } \\
\text { and can } \\
\text { not see } \\
\text { goal }\end{array}$ & Total \\
\hline $\begin{array}{l}\text { Krislet } \\
\text { expert }\end{array}$ & 792 & 2227 & 60 & 71 & 52 & 3131 \\
\hline $\begin{array}{l}\text { MLP } \\
\text { learner }\end{array}$ & 808 & 2138 & 50 & 117 & 49 & 3045 \\
\hline Total & 1600 & 4365 & 110 & 188 & 101 & 6176 \\
\hline
\end{tabular}

Table 4.3: Example environment state frequencies of the Krislet expert and the MLP agent in the RoboCup soccer domain. 


$$
\begin{aligned}
m_{\text {learner, ball visible }} & =3045 \times \frac{1600}{6176} \\
m_{\text {learner, ball visible }} & \approx 789 \\
x_{\text {learner, ball visible }} & =808 \\
\frac{\left(x_{\text {learner, ball visible }}-m_{\text {learner, ball visible }}\right)^{2}}{m_{\text {learner, ball visible }}} & =\frac{(808-789)^{2}}{789} \\
\frac{\left(x_{\text {learner, ball visible }}-m_{\text {learner, ball visible }}\right)^{2}}{m_{\text {learner, ball visible }}} & \approx 0.458
\end{aligned}
$$

The sum of the previous quantity over all cells in Table 4.3 provides the $\chi^{2}$ statistic which is correlated to the distance between the environment state probability distribution of the expert and the agent's trajectories:

$$
\chi_{P(S)}^{2}=\sum_{\text {agent, state }} \frac{\left(x_{\text {agent, state }}-m_{\text {agent, state }}\right)^{2}}{m_{\text {agent, state }}} \approx 14.0
$$

\subsection{Conclusion}

In this section, we remodelled the RoboCup soccer environment state vector of our agents and provided experts that test different dimensions of state-based behavior. This helped us establish a complex benchmark where state-based imitation learning techniques can be tested in a more realistic environment. We also proposed the use of a LSTM network instead of current state-based imitation learning techniques, to be able to learn the function $F$ that maps a run to a hidden internal state of an expert without making an assumption (refer to Section 4.3). Moreover, we proposed new metrics that we will use in the next chapter to compare the performance of LSTMs against state-of-the-art state-based imitation learning techniques in both the discrete vacuum cleaner domain and the continuous RoboCup soccer domain. Lastly, we 
discussed all the benchmarks, techniques, testing methodologies, and metrics that we will use in our experiments.

In the next chapter, we will investigate the effect of DAgger on the imitation performance of CBR, TB, LSTM and MLP techniques. We will show how DAgger improves the performance of LSTM, MLP, and TB, but detriments the performance of CBR techniques that use past actions. We will also show how the LSTM network outperformed other techniques at imitating our state-based experts in the continuous RoboCup soccer domain. Additionally, we will validate our online testing methodology by comparing the results we obtained in the vacuum cleaner domain to those obtained using the offline testing methodology adopted by other research in the field of imitation learning. We will show how our online testing method is reliable whereas the offline testing method does not always provide results that correspond to the performance of the agents. We will also show how LSTM networks are not always better than state-of-the-art techniques at imitating the experts in the discrete vacuum cleaner domain. 


\section{Chapter 5}

\section{Experiments}

\subsection{Introduction}

We divided this chapter into four main sections that each correspond with an experiment we conducted. In the first section, we perform a preliminary experiment to select which Case-Based Reasoning (CBR) similarity metric to use in the following experiments. The goal of this experiment is to reduce the number of imitation learning agents we will compare to our proposed techniques, as there are many CBR similarity metrics (refer to Section 3.3.5 for the list of metrics).

In the following section, we compare the performance of each imitation learning technique that we will use in the RoboCup soccer domain, before and after the application of Dataset Aggregation (DAgger). The objective of this experiment is to test how DAgger impacts the performance of different imitation learning techniques.

In the third section of this chapter, we perform a test comparing each imitation learning technique in the continuous RoboCup soccer domain. The purpose of this experiment is to identify which imitation learning technique performs best at imitating 
each RoboCup soccer expert. Hence, this section is divided into 6 subsections pertaining to each RoboCup soccer expert from Section 4.2.2. The source code used for all the RoboCup soccer experiments, including the preliminary experiment and DAgger, can be found in the following repositories: github.com/MohamedZalat/robocup-soccer, and github.com/MohamedZalat/Krislet_jLOAF.

In the last section of this chapter, we perform 2 experiments in the vacuum cleaner domain: (1) we compare results obtained by the offline testing methodology used by Gunaratne et al. [4] to the results obtained using our online testing methodology, and (2) we test each imitation learning technique in the discrete vacuum cleaner domain. The objective of the first experiment is to show how the offline testing method does not provide results that correspond with the real world test of the imitation learning agents. Whereas, the objective of the second experiment is to identify which techniques perform best at imitating the experts in the vacuum cleaner domain. It will also be interesting to investigate if those techniques are different from those that perform well in the RoboCup soccer domain. Similar to the third section of this chapter, each experiment is divided into paragraphs pertaining to each vacuum cleaner expert. The source code used for all the vacuum cleaner experiments can be found at github.com/MohamedZalat/LFO-Simulator. 


\subsection{Preliminary Experiment: CBR Similarity Met- rics in RoboCup Soccer}

\subsubsection{Introduction}

There are many different CBR similarity metrics we described in Section 3.3.5. In order to limit the number of imitation learning techniques that we will use in future experiments, we will select the CBR similarity metric that performs better on average compared to the rest. There are only three CBR similarity metrics that can work for both continuous and discrete domains. Namely, those metrics are the n-ordered similarity metric, the ordered similarity metric and the weighted similarity metric.

The three similarity metrics are almost identical, with a small variation between each other. As we mentioned in Section 3.3.5, the n-ordered similarity metric computes the similarity of the last $n$ environment states and actions in the trajectory of the agent to that in its training examples (i.e. learnt trajectories). The ordered similarity metric computes the same similarity; however, it uses the entire trajectory of the agent instead of the last $n$ elements. Lastly, the weighted similarity is identical to the ordered similarity; however, it weighs the first 10 elements in the agent's trajectory in an exponentially decreasing manner while assigning each element in the remainder of the trajectory a weight of 1 .

Since the weighted similarity metric is just an improved version of the ordered similarity metric (in the case of experts that base most of their decisions on more recent events), we decided to exclude the ordered similarity metric from this experiment. Hence, we use the n-ordered similarity metric and the weighted similarity metric in this experiment. 
We used the RoboCup soccer domain to test which of those two metrics perform better on average, since this domain is continuous and more complex than the vacuum cleaner domain. Moreover, we have two experts in this domain that can give us insight on how each metric performs at imitating state-based behavior that requires (1) long-term memory and (2) short-term memory. As we recall from Section 4.2.2, the Switch expert switches the direction it turns in when looking for the ball after kicking the ball. This means in the worst case scenario, where the agent never lost sight of the ball, it has to recall its entire trajectory to identify which direction it should turn in when it loses sight of the ball. On the other hand, the Offense expert dashes to the ball without regard to its angle from the ball 20 times before aligning its direction with the ball. Hence, it should suffice to remember the last 80 elements in its trajectory (i.e. the last 40 environment states and actions) because it may lose sight of the ball and turn instead of dash (just to have the ball in its field of view). Since we know how each expert should behave in this experiment and all future experiments of this thesis, it is normal to have expectations on how the imitation learning agents should behave if they are imitating an expert correctly. Thus, we will use those expectations when qualitatively assessing the behavior of each imitation learning agent as in the work of Tîrnăucă et al. [3].

In this experiment, we will use the Switch expert as an example of a state-based expert that requires long-term memory and the Offense expert as an example a state-based expert that requires short-term memory. Since CBR accounts for missing features when computing similarities, we do not include the features that indicate the existence of an object in its field of view (i.e. the visibility of the goal, its own goal and the ball from Section 4.2.1.1).

We set the hyper-parameter $n$ of the n-ordered similarity to 1000 in the case of 
the Switch expert since the Switch expert requires long-term memory and to 80 in the case of the Offense expert as it requires short-term memory. The training set of both the weighted and the n-ordered similarity were obtained from a match of the expert playing against itself in a full match of RoboCup soccer. This resulted in a training trajectory that is 3700 cases long (where a case is an environment state and action pair). Note that the training sets for this experiment were not obtained using DAgger because in the next experiment we will show how DAgger negatively affects the performance of CBR.

The weighted similarity metric considers the entire trajectory. However, the trajectories of RoboCup soccer agents are very long, which will make the weight of the latest 10 elements insignificant in comparison to the rest of the elements in the trajectory. Hence, we expect the weighted similarity metric to perform worse at imitating the Offense expert compared to the n-ordered similarity metric (which only considers the past $n=80$ elements in its trajectory). We also expect that the weighted similarity metric will perform very similarly to the n-ordered similarity metric in the case of the Switch expert. This is because $n$ is set to a large number $(n=1000)$ when imitating the Switch expert, and the weighting of the last 10 elements that the weighted similarity performs is not significant enough in comparison to the remainder of the agent's trajectory due to how long the trajectory is. Hence, we expect that our results will show that the n-ordered similarity metric is going to be our similarity metric of choice when comparing CBR to other techniques in the experiments we will perform in the remainder of this chapter.

\subsubsection{Results and Discussion}




\begin{tabular}{|c|c|c|c|c|c|c|c|c|}
\hline Agent & $\begin{array}{l}\text { Macro } \\
\text { F1 }\end{array}$ & \multicolumn{2}{|c|}{$\begin{array}{l}\text { Monte } \\
\text { d Carlo }\end{array}$} & $\chi_{P(S)}^{2}$ & $\chi_{P(A)}^{2}$ & $\chi_{P\left(S_{t}, S\right.}^{2}$ & $\left.{ }_{1}\right) \chi_{P\left(A_{t}, A\right.}^{2}$ & ${ }_{1} \chi_{P(A, S)}^{2}$ \\
\hline $\begin{array}{l}\text { N- } \\
\text { Ordered } \\
(n= \\
1000)\end{array}$ & 0.23 & 0.36 & 5.95 & 1545 & 3444 & 1831 & 4852 & 5036 \\
\hline Weighted & 0.29 & 0.3 & 6.51 & 1577 & 2921 & 1875 & 4046 & 5623 \\
\hline
\end{tabular}

Table 5.1: The results of the n-ordered similarity and weighted similarity CBR agents at imitating the Switch expert.

Switch Expert In Table 5.1, we present the results of our experiments using the Switch expert. Firstly, we noted that neither the n-ordered nor the weighted similarity metrics were remotely close to the Switch expert's behavior. This is due to how those similarity metrics choose which action to perform. The Switch expert uses the latest environment state when deciding which action to take in most cases, unless it can not see the ball. In the case where it can not see the ball, the expert decides which direction to turn in based on its hidden internal state. Notice how, in this scenario, the current environment state is the first thing the expert considers before recalling its hidden internal state when selecting which action to perform. On the other hand, the n-ordered similarity metric weights the latest 1000 elements in its trajectory equally when considering which action to take, making it select the incorrect action the majority of the time. Similarly, due to how long the trajectory of the weighted similarity agent is, weighting the 10 latest elements of the trajectory does not significantly impact its performance.

From the results of our experiment in Table 5.1, we see that not all the performance metrics agree with each other on which CBR metric performed better at imitating the 
Switch expert. It was also difficult to discern which agent imitated the Switch expert by qualitatively observing the two agents.

Since the difference in performance between the weighted similarity metric and the n-ordered similarity metric at imitating the Switch expert was not significant enough to conclude which one performs better than the other, we will use the results of the Offense expert to conclude which CBR similarity metric we will be using in the next experiments.

\begin{tabular}{|c|c|c|c|c|c|c|c|c|}
\hline \multirow{2}{*}{ Agent } & \multirow{2}{*}{$\begin{array}{l}\text { Macro } \\
\text { F1 }\end{array}$} & \multicolumn{2}{|c|}{$\begin{array}{l}\text { Monte } \\
\text { d Carlo }\end{array}$} & \multirow{2}{*}{$\chi_{P(S)}^{2}$} & \multirow{2}{*}{$\chi_{P(A)}^{2}$} & \multirow{2}{*}{\multicolumn{3}{|c|}{$\chi_{P\left(S_{t}, S_{t-1}\right)}^{2} \chi_{P\left(A_{t}, A_{t-1}\right)}^{2} \chi_{P(A, S)}^{2}$}} \\
\hline & & F1 & $\begin{array}{l}\text { Dis- } \\
\text { tance }\end{array}$ & & & & & \\
\hline $\begin{array}{l}\text { N- } \\
\text { Ordered } \\
(n= \\
80)\end{array}$ & 0.19 & 0.15 & 4.01 & 975 & 62.2 & 1733 & 1026 & 4501 \\
\hline \multicolumn{2}{|c|}{ Weighted 0.11} & 0.06 & 6.41 & 3010 & 1738 & 3384 & 2868 & 5238 \\
\hline
\end{tabular}

Table 5.2: The results of the n-ordered similarity and weighted similarity CBR agents at imitating the Offense expert.

Offense Expert In Table 5.2, we present the results of the n-ordered and weighted agents when imitating the Offense expert. Similar to the Switch expert, we found that neither the n-ordered similarity metric nor the weighted similarity metric imitated the Offense expert adequately when observing them play a match of RoboCup soccer. This is due to the same reasons we previously mentioned when discussing the results of the Switch expert: this expert weights the current environment state heavily when deciding which action to take, even in situations where it requires recalling its past.

However, this time the n-ordered similarity metric imitated the Offense expert noticeably better than the weighted similarity metric. This is because the n-ordered 
similarity metric had a much smaller hyper-parameter $n$, thus giving the most recent environment state a larger contribution to the total similarity when selecting which action to take. Instead, the weighted similarity metric is still using the entire trajectory when computing the similarity. All our results in Table 5.2, agree with this observable difference in performance. For those reasons, we will use the n-ordered similarity metric, as our CBR similarity metric when comparing CBR to other imitation learning techniques in the remainder of this Chapter.

\subsubsection{Conclusion}

Our experiment concluded that the n-ordered similarity metric performs similarly to the weighted similarity metric in cases where a long-term memory is required (i.e. a large $n$ ) and better in cases where short-term memory is required (i.e. a small $n$ ) as we expected. Hence, we will be using the n-ordered similarity metric in our next experiments as our CBR candidate. On a side note, we also observed how our proposed performance metrics gave us insight on the observable difference in

performance between the n-ordered similarity and the weighted similarity at imitating the Switch expert; which all other metrics failed to provide.

\subsection{The Effect of DAgger on Imitation Learning Techniques}

Recall from Section 2.5.1, that DAgger is an algorithm used to augment the training set of an imitation learning agent, with examples provided by feedback from the expert while the learning agent is running. The objective of DAgger is to provide a training set that has an environment state distribution identical to that observed by the agent 
during run-time. This is because most machine learning models make an assumption that the input of the model during run-time is independent and identically distributed to the inputs of its training set. While this is the case in many machine learning problems, it is not the case for imitation learning. In imitation learning, any incorrect action will result in a different environment state compared to the expert; hence, the environment state distribution of the imitation learning agent during run-time differs from the environment state distribution of its training set.

while DAgger aims to minimize the difference between the environment state distribution of an agent's training set and its run-time distribution, it does not minimize the difference between the action distribution of the training set and its run-time action distribution. Since the CBR similarity metric we considered uses trajectories rather than the current environment state of the agent to select an action, and trajectories include actions, CBR will take many time step before it selects the correct action suggested by the expert. However, the actions added to the training trajectory by DAgger are different from the actions selected by the n-ordered CBR similarity metric during its run. This results in the training trajectory augmented by DAgger to diverge away from the run-time trajectory of the state-based CBR agent. Therefore, this should result in the state-based CBR agent performing worse after DAgger than before DAgger.

On the other hand, Multi-Layer Perceptron (MLP), Long Short-Term Memory (LSTM), and Temporal Backtracking (TB) have bias to the latest environment state. The MLP only considers the most recent environment state. The LSTM only considers the current environment state and past environment states through its hidden internal state (no actions). While TB may consider past actions as well as past environment states, it has a bias to the most recent environment state(s). Moreover, TB can be 
configured to exclude considering the actions in its trajectory by setting its solution threshold to 0 (refer to Section 3.3.2). Hence, we expect that DAgger will improve MLP, LSTM and TB.

To test our theory, we will perform an experiment with the n-ordered CBR similarity metric, TB, MLP, and LSTM in the RoboCup soccer domain comparing their performance before and after the application of DAgger. We chose the RoboCup soccer domain as it provides a complex benchmark that is realistic. We will also use the same experts from the previous experiment: the Switch expert as an example of state-based behavior that requires a long-term memory, and the Offense expert as an example of state-based behavior that requires a short-term memory.

In the first section of this experiment, we will describe our hyper-parameters and our training method for each technique. In the section that follows, we will discuss and report the results of our DAgger experiment. We divided that section into paragraphs that pertain to each technique and its results at imitating each expert with and without DAgger. Lastly, we will conclude whether the experiment confirmed our theory or not.

\subsubsection{DAgger Experiment Setup}

We set the hyper-parameter $n$ of the n-ordered similarity agent to $n=1000$ for the Switch expert, and to $n=80$ for the Offense expert (same as Section 5.2). We also set the problem threshold of TB to $P T=0.99$ and the solution threshold to $S T=0$, so that it does not consider actions when it is backtracking.

Similar to the previous experiment, the techniques trained without DAgger use a training set collected from the expert in a full match against itself (i.e. a trajectory of approximately 3700 cases). The techniques trained with DAgger augment this training set by running against the expert they are imitating in a match of soccer. 
We apply DAgger differently in the case of CBR and TB as they employ a lazy training method. At each time step, we perform the action the agent chose before augmenting its training set with the current environment state and the action the expert would have performed. This means that at every time step, before performing an action, the agent is "trained" on all the time steps before the current step. Since both of these techniques employ a lazy training technique and we need them to be responsive at run-time, we opted to augment their initial training set by applying DAgger for only 2 matches against the expert they are imitating. This resulted in their final training set containing approximately 10000 cases.

In the case of MLP and LSTM, they do not employ lazy training. Hence, we initially train them for 200 epochs on their current training set before the start of each soccer match. Then, we retrain them for 10 epochs every 10 time steps in the match; where each epoch involves training the network over the new augmented training set as one large trajectory (with 10 new cases). This was repeated till we observed little to no improvement in the performance of the techniques at imitating each expert. This resulted in 47647 cases and 51972 cases in the training set of the MLP imitating the Switch and Offense experts respectively. In the context of the LSTM, this resulted in 37910 cases and 27606 cases in its training set when imitating the Switch and Offense experts respectively.

Both the MLP and LSTM models were trained using a learning step of $\lambda=0.0001$ by minimizing the cross entropy loss using the Adam optimizer.

\subsubsection{Results and Discussion}

N-Ordered In Table 5.3, we present the result of the n-ordered CBR similarity metric at imitating the Switch and Offense experts before and after the application 


\begin{tabular}{|c|c|c|c|c|c|c|c|c|}
\hline Expert & $\begin{array}{l}\text { Macro } \\
\text { F1 }\end{array}$ & \multicolumn{2}{|c|}{$\begin{array}{l}\text { Monte } \\
\text { dCarlo }\end{array}$} & $\chi_{P(S)}^{2}$ & $\chi_{P(A)}^{2}$ & \multicolumn{3}{|c|}{$\chi_{P\left(S_{t}, S_{t-1}\right)}^{2} \chi_{P\left(A_{t}, A_{t-1}\right)}^{2} \chi_{P(A, S)}^{2}$} \\
\hline $\begin{array}{l}\text { Switch } \\
(n= \\
1000, \\
\text { DAg- } \\
\text { ger=No) }\end{array}$ & 0.23 & 0.36 & 5.95 & 1545 & 3444 & 1831 & 4852 & 5036 \\
\hline $\begin{array}{l}\text { Switch } \\
(n= \\
1000, \\
\text { DAg- } \\
\text { ger=Yes })\end{array}$ & 0.21 & 0.13 & 7.08 & 1893 & 2920 & 2103 & 4132 & 5155 \\
\hline $\begin{array}{l}\text { Offense } \\
(n= \\
80, \\
\text { DAg- } \\
\text { ger }=\text { No) }\end{array}$ & 0.19 & 0.15 & 4.01 & 975 & 62.2 & 1733 & 1026 & 4501 \\
\hline $\begin{array}{l}\text { Offense } \\
(n= \\
80, \\
\text { DAg- } \\
\text { ger=Yes })\end{array}$ & 0.13 & 0.05 & 6.73 & 3745 & 1888 & 4029 & 3090 & 5084 \\
\hline
\end{tabular}

Table 5.3: The results of the n-ordered similarity at imitating the experts before and after the application of DAgger.

of DAgger. Just as we expected, there was a noticeable decline in the performance of the n-ordered similarity metric after the application of DAgger when examining them play against the expert. This trend was consistent between both the experts we used. Moreover, our quantitative results in Table 5.3 are consistent with the observed behavior. However, the $P(A)$ and $P\left(A_{t}, A_{t-1}\right)$ distributions of the n-ordered agent after DAgger seem to be closer to the distributions of the Switch expert. This is expected as we mentioned in Section 4.4.1 that we expect those two distributions to 
not always correlate with the qualitative behavior of the agent relative to the expert.

Therefore, we can safely conclude from our experiment with the n-ordered CBR metric, that it does not synergize well with DAgger, as we predicted.

\begin{tabular}{|c|c|c|c|c|c|c|c|c|}
\hline \multirow{2}{*}{$\begin{array}{l}\text { Expert } \\
\text { Switch } \\
\text { (DAg- } \\
\text { ger } \\
=\mathrm{No} \text { ) }\end{array}$} & \multirow{2}{*}{$\begin{array}{l}\text { Macro } \\
\text { F1 } \\
0.36\end{array}$} & \multicolumn{2}{|c|}{$\begin{array}{lc} & \text { Monte } \\
\text { Weighted Carlo } \\
\text { F1 } & \text { Dis- } \\
& \text { tance }\end{array}$} & $\chi_{P(S)}^{2}$ & $\chi_{P(A)}^{2}$ & \multicolumn{3}{|c|}{$\chi_{P\left(S_{t}, S_{t-1}\right.}^{2} \chi_{P\left(A_{t}, A_{t-1}\right)}^{2} \chi_{P(A, S)}^{2}$} \\
\hline & & 0.50 & 4.18 & 665 & 202 & 995 & 324 & 2237 \\
\hline $\begin{array}{l}\text { Switch } \\
\text { (DAg- } \\
\text { ger=Yes) }\end{array}$ & 0.55 & 0.71 & 3.02 & 677 & 631 & 824 & 805 & 1539 \\
\hline $\begin{array}{l}\text { Offense } \\
(\mathrm{DAg}- \\
\text { ger }=\mathrm{No})\end{array}$ & 0.47 & 0.56 & 3.05 & 174 & 194 & 359 & 295 & 1423 \\
\hline $\begin{array}{l}\text { Offense } \\
\text { (DAg- } \\
\text { ger=Yes) }\end{array}$ & 0.68 & 0.75 & 2.08 & 67.4 & 23.3 & 97.4 & 41.5 & 551 \\
\hline
\end{tabular}

Table 5.4: The results of TB at imitating the experts before and after the application of DAgger.

Temporal Backtracking In Table 5.4, we present the results of our experiment with TB. This time, we saw an improvement after we applied DAgger in both the Offense and Switch experts. However, in the case of the Switch expert, this improvement was much less noticeable. This is due to the augmented training set resulting in more cases of a turn in one direction than the other. As DAgger was executed when imitating the Switch expert, the TB agent often alternated its turn direction after every turn when it lost sight of the ball. This is due to how TB prioritizes the comparison of the 
most recent environment states to obtain a set of non-disagreeing actions and the way DAgger creates a training trajectory that is displaced by one environment state from the run trajectory of the agent. This resulted in a conflicting turn direction between its past training trajectories and the new DAgger trajectory that is misaligned by one environment state. Thus, the agent keeps turning in alternating direction that result in alternating between two environment states.

Our proposed metrics highlight this disparity between consistently turning in the correct direction after the application of DAgger. The $P(A)$ and $P\left(A_{t}, A_{t-1}\right)$ distributions are further away from the Switch expert after applying DAgger, because it had many situations where it changed its turn direction. This is due to setting the solution threshold of TB to 0 which makes TB ignore actions in the trajectory. Despite this issue, the TB agent still performed better at imitating the Switch expert after the application of DAgger.

We can safely conclude from both our qualitative and quantitative results in Table 5.4, that DAgger improved the performance of TB at imitating the experts we used in this experiment as we predicted earlier in this section.

Multi-Layer Perceptron In Table 5.5, we present the results of our experiment when using the MLP agent. Firstly, the MLP agent is inherently incapable of imitating state-based experts since it only considers the current environment state of the agent without regard to previous environment states. Hence, it is no surprise that it was unable to reproduce the state-based behavior of neither the Switch nor the Offense experts. It was unable to identify which direction to turn in when it lost sight of the ball when imitating the Switch expert. Instead, it constantly alternated the direction it was turning in when it lost sight of the ball. In the case of the Offense expert, it 


\begin{tabular}{|c|c|c|c|c|c|c|c|c|}
\hline Expert & $\begin{array}{l}\text { Macro } \\
\text { F1 }\end{array}$ & \multicolumn{2}{|c|}{$\begin{array}{l}\text { Monte } \\
\text { d Carlo }\end{array}$} & $\chi_{P(S)}^{2}$ & $\chi_{P(A)}^{2}$ & \multicolumn{3}{|c|}{$\chi_{P\left(S_{t}, S_{t-1}\right)}^{2} \chi_{P\left(A_{t}, A_{t-1}\right)}^{2} \chi_{P(A, S)}^{2}$} \\
\hline $\begin{array}{l}\text { Switch } \\
\text { (DAg- } \\
\text { ger } \\
=\mathrm{No} \text { ) }\end{array}$ & 0.32 & 0.31 & 5.94 & 1589 & 239 & 2508 & 712 & 3921 \\
\hline $\begin{array}{l}\text { Switch } \\
\text { (DAg- } \\
\text { ger=Yes) }\end{array}$ & 0.58 & 0.78 & 2.50 & 276 & 336 & 366 & 739 & 816 \\
\hline $\begin{array}{l}\text { Offense } \\
(\mathrm{DAg}- \\
\text { ger }=\mathrm{No})\end{array}$ & 0.28 & 0.21 & 5.10 & 286 & 794 & 1045 & 1773 & 3955 \\
\hline $\begin{array}{l}\text { Offense } \\
(\mathrm{DAg}- \\
\text { ger=Yes) }\end{array}$ & 0.70 & 0.79 & 2.12 & 42.7 & 136 & 155 & 396 & 436 \\
\hline
\end{tabular}

Table 5.5: The performance metrics of MLP at imitating the experts before and after the application of DAgger.

was unable to identify when it should dash and when it should align with the ball. So, it resorted to dashing a majority of the time even when it should turn to the ball. Despite this, it was able to perform the actions that require no state information (in both experts) without many problems.

Just as we hypothesized earlier, DAgger had a significant positive impact on both the qualitative and quantitative performance of the MLP at imitating both experts. Before DAgger, the MLP agent often attempted to kick the ball before it was close enough to the ball and in cases where it cannot see the ball. After DAgger was applied, the MLP agent was able to correctly deal with those scenarios in both experts: it dashed when it was not close to the ball and kicked the ball only when it can see both the ball and the goal. Therefore, we can conclude that this experiment confirmed 
what we expected when applying DAgger to MLP: DAgger improved the performance of MLP at imitating the experts we used in this experiment.

\begin{tabular}{|c|c|c|c|c|c|c|c|c|}
\hline Expert & $\begin{array}{l}\text { Macro } \\
\text { F1 }\end{array}$ & \multicolumn{2}{|c|}{$\begin{array}{l}\text { Monte } \\
\text { d Carlo }\end{array}$} & $\chi_{P(S)}^{2}$ & $\chi_{P(A)}^{2}$ & $\chi_{P\left(S_{t}, S_{t}\right.}^{2}$ & $\left.{ }_{1}\right) \chi_{P\left(A_{t}, A\right.}^{2}$ & $\chi_{P(A, S)}^{2}$ \\
\hline $\begin{array}{l}\text { Switch } \\
\text { (DAg- } \\
\text { ger } \\
=\mathrm{No} \text { ) }\end{array}$ & 0.23 & 0.17 & 6.56 & 1973 & 767 & 3010 & 1502 & 4723 \\
\hline $\begin{array}{l}\text { Switch } \\
\text { (DAg- } \\
\text { ger=Yes) }\end{array}$ & 0.77 & 0.94 & 1.21 & 90.0 & 39.1 & 131 & 80.9 & 183 \\
\hline $\begin{array}{l}\text { Offense } \\
(\mathrm{DAg}- \\
\text { ger }=\mathrm{No})\end{array}$ & 0.32 & 0.25 & 3.49 & 1668 & 359 & 2292 & 822 & 3124 \\
\hline $\begin{array}{l}\text { Offense } \\
(\mathrm{DAg}- \\
\text { ger =Yes })\end{array}$ & 0.70 & 0.79 & 2.26 & 135 & 12.2 & 173 & 84.2 & 505 \\
\hline
\end{tabular}

Table 5.6: The performance metrics of LSTM at imitating the experts before and after the application of DAgger.

Long Short-Term Memory We present the results of the experiment with LSTM in Table 5.6. We expected the LSTM to perform well at imitating both the Switch and Offense experts. While it performed much better than any other technique at imitating both experts after DAgger was applied, it performed much worse than many other techniques before the application of DAgger.

Before the application of DAgger, the LSTM failed to imitate the experts in simple scenarios that involve no information from past environment states, such as kicking the ball when it is close to the ball. It often dashed instead of kicking the ball and it 
often kicked the ball when it is not close enough to the ball.

After the application of DAgger, the LSTM agent was not only able to perform the basic behaviors that require no state information in both the Switch and Offense experts; it was also able to identify which direction to turn in, when imitating the Switch expert, and it was able to discern the situations where it should dash versus align itself with the ball in the case of the Offense expert. However, it was not always perfect especially in the case of the Offense expert. It often continued dashing when it should align with the ball, even after the 20 dashes have been performed. This behavior was observed until it lost sight of the ball where it reverted to the correct behavior; that is, aligning with the ball before dashing.

Our qualitative analysis of the effect of DAgger on LSTM, and our results in Table 5.6 both agree that the DAgger had a very significant positive impact on the performance of the LSTM when imitating the experts we used in our experiments. This is inline with what we predicted before performing this experiment.

\subsubsection{Conclusion}

Our results of this experiment concluded that our hypothesis on the effect of DAgger on each imitation learning technique is likely to be correct. All the experts we used in this experiment gave us results that indicated that DAgger negatively impacted the performance of the n-ordered similarity CBR. On the other hand, our results from this experiment indicated that DAgger improved the imitation performance of TB, MLP, and LSTM. 


\subsection{RoboCup Soccer Experiments}

\subsubsection{Introduction}

In the previous chapter, we modelled the RoboCup soccer environment state vector to preserve its continuous nature. We also designed RoboCup soccer experts that test a variety of state-based behavior and included an expert that tests reactive behavior. Recall from Section 4.2.2, the Krislet expert [35] is a simple expert that chases the ball and kicks it to the enemy goal once it gets close to the ball. This expert was designed to test the ability of each imitation learning technique to capture a simple reactive behavior (i.e. behavior that requires no information from past environment states or actions). The Switch expert is a state-based expert (i.e. an expert that requires information from past environment states and/or actions) that switches the direction it turns when looking for the ball every time it kicks the ball. This is an example of an expert that requires a long-term memory if the agent never lost sight of the ball. The Offense expert is another state-based expert that instead requires a short-term memory. It relies on counting the number of times it dashed after performing a kick action to properly switch its hidden internal state.

The Pro, Goal, and Pro Goal experts are three experts that rely on remembering a feature(s) of an object they lost sight of to determine which direction to turn in. The Pro expert relies on remembering a feature from the short-term; particularly, the direction of the ball to turn in the correct direction when it loses sight of it. The Goal expert relies on remembering a feature from the long-term: the last direction of the enemy goal, to turn in the correct direction when looking for the goal. Lastly, the Pro Goal expert combines those two experts into one: it remembers both the last direction of the goal and the last direction of the ball, to turn in the correct direction when it 
loses sight of either object.

In this experiment we will test the performance of MLP, LSTM, TB, and n-ordered similarity metric at imitating each of the experts in RoboCup soccer. The purpose of this experiment is to identify which imitation learning technique is best at imitating each expert, and if the LSTM agent is universally better than the other agents at imitating all the experts we used. In the first subsection of this experiment, we will list the configurations of our hyper-parameters for each technique and the training set size of each agent. In the six subsections that follow, we present the results of imitating each expert, starting with the reactive Krislet expert, and ending with the state-based Pro Goal expert. In the last subsection of this experiment, we conclude on which imitation learning technique performed best at imitating our RoboCup soccer experts.

\subsubsection{RoboCup Soccer Experiment Setup}

In order to be consistent with all techniques, we decided to collect the training set of all the techniques we use in this experiment using DAgger. We obtain the training set using the same method described in the previous experiment for all our agents (refer to Section 5.3.1). We also set the hyper-parameters to the same hyper-parameters we used in that experiment.

Recall from Section 5.3.1, we set the learning step of both the MLP and LSTM agents to $\lambda=0.0001$. We also used the Adam optimizer to minimize the cross entropy loss. The training set size of each expert the MLP imitates is presented in Table 5.7, and the training set sizes of the LSTM are presented in Table 5.8. Note that we made sure that the training set size of the MLP is larger than the training set size of the LSTM to ensure the MLP is trained to its maximum potential. Since the MLP cannot 


\begin{tabular}{ll}
\hline Expert & Training set size \\
\hline Krislet & 85385 \\
Switch & 47674 \\
Offense & 51972 \\
Pro & 116321 \\
Goal & 265029 \\
Pro Goal & 64165 \\
\hline
\end{tabular}

Table 5.7: The final training set size used by the MLP agent to imitate each expert.

\begin{tabular}{ll}
\hline Expert & Training set size \\
\hline Krislet & 12676 \\
Switch & 37910 \\
Offense & 27606 \\
Pro & 34009 \\
Goal & 30219 \\
Pro Goal & 22260 \\
\hline
\end{tabular}

Table 5.8: The final training set size used by the LSTM agent to imitate each expert.

imitate state-based behavior, increasing the size of its training set should not make it perform better than the LSTM at imitating state-based behavior. If our results do not suggest this, then this indicates a fault in our experiment.

Similar to our experiment in Section 5.3, we collected a training set size of approximately 10000 cases each using DAgger for TB and the n-ordered similarity metric. This is because collecting more cases will impact their run-time responsiveness as they are techniques that employ lazy training.

In the context of $\mathrm{TB}$, we used a solution threshold of 0 to ignore actions when backtracking and a problem threshold of 0.99. The purpose of setting the solution threshold to 0 is to benefit from the application of DAgger as mentioned in Section 5.3. 
We set the hyper-parameter $n$ of the n-ordered similarity depending on how many past environment states it needs to recall for the expert its imitating. For the Krislet expert, it only needs the most recent environment state; hence, we set $n=1$. For the Switch expert, it may need to recall its entire trajectory in the worst case scenario; hence, we set $n$ to a large number $n=1000$. In the case of the Offense expert, it needs to recall the last 20 environment states or slightly more; hence we set $n=80$ to be conservative (last 40 environment states and 40 actions). In the case of the Pro expert, it needs to recall the last 5 environment states on average; hence, we set $n=10$. Similarly, the Goal expert needs to recall the last 200 environment states on average; so we set $n=400$. Lastly, we set $n=400$ for the Pro Goal expert as it combines the Goal expert and the Pro expert.

\subsubsection{Krislet Expert Results}

\begin{tabular}{lllllllll}
\hline \multicolumn{7}{c}{ Agent } & Macro & Weighted Carlo \\
& F1 & F1 & $\begin{array}{c}\text { Dis- } \\
\text { tance }\end{array}$ & $\chi_{P(S)}^{2}$ & $\chi_{P(A)}^{2}$ & $\chi_{P\left(S_{t}, S_{t-1}\right)}^{2} \chi_{P\left(A_{t}, A_{t-1}\right)}^{2} \chi_{P(A, S)}^{2}$ \\
\hline MLP & 0.92 & 0.96 & 0.987 & 14.0 & 9.24 & 39.2 & 33.0 & 94.7 \\
LSTM & 0.82 & 0.94 & 1.16 & 56.3 & 4.43 & 155 & 62.2 & 168 \\
TB & 0.67 & 0.76 & 2.58 & 123 & 16.9 & 169 & 54.9 & 1011 \\
N- & & & & & & & & \\
$\begin{array}{l}\text { Ordered } \\
(n=\end{array}$ & 0.65 & 0.75 & 2.66 & 256 & 97.5 & 432 & 157 & 1041 \\
$1)$ & & & & & & & & \\
\hline
\end{tabular}

Table 5.9: The results of imitating the Krislet expert.

We present the results of imitating the reactive Krislet expert in Table 5.9. From the behavior we observed, we found that the MLP was the closest agent to the behavior 
of the Krislet expert. While the LSTM was a close second, it seemed to have learnt a coincidental sequence of action that the Krislet expert commonly performed. The LSTM occasionally turned to the ball once after seeing the ball before performing a sequence of dashes (even when it should turn to the ball). This resulted in the LSTM performing slightly worse than the MLP. However, the LSTM still performed better than any of the other techniques designed to imitate state-based behavior. This was evident in our quantitative results in Table 5.9. The TB and n-ordered similarity seemed to perform very similarly when imitating the Krislet expert.

Our results for this experiment indicate that while state-based imitation learning techniques may be good at imitating state-based behavior, they may not be as good at imitating reactive experts as techniques that are limited to reactive behavior. We can not conclude that the LSTM agent is the best state-based imitation learning technique at imitating reactive behavior from this experiment; because it was trained using a much larger training set compared to the other state-based imitation learning techniques.

\subsubsection{Switch Expert Results}

We present the results of our experiments with the Switch expert in Table 5.10. Out of all the techniques, the only technique that adequately imitated the Switch expert was the LSTM expert. It was often able to identify the correct direction it should turn in whenever it lost sight of the ball. Even in the occasional situations where it did not correctly identify the direction it should turn in, it consistently turned in the direction it chose. This behavior was very close to the behavior of the expert.

On the other hand, the MLP and TB agents often got stuck alternating the direction they turn in when they lost sight of the ball. This behavior also seemed to 


\begin{tabular}{|c|c|c|c|c|c|c|c|c|}
\hline \multirow{3}{*}{$\begin{array}{l}\text { Agent } \\
\text { MLP }\end{array}$} & \multirow{3}{*}{$\begin{array}{l}\text { Macro } \\
\text { F1 } \\
0.58\end{array}$} & \multirow{2}{*}{\multicolumn{2}{|c|}{$\begin{array}{c}\text { Monte } \\
\text { d Carlo } \\
\text { Dis- } \\
\text { tance }\end{array}$}} & \multirow{3}{*}{$\begin{array}{l}\chi_{P(S)}^{2} \\
276\end{array}$} & \multirow{3}{*}{$\begin{array}{l}\chi_{P(A)}^{2} \\
336\end{array}$} & \multirow{2}{*}{\multicolumn{3}{|c|}{$\chi_{P\left(S_{t}, S_{t-1}\right)}^{2} \chi_{P\left(A_{t}, A_{t-1}\right)}^{2} \chi_{P(A, S)}^{2}$}} \\
\hline & & & & & & & & \\
\hline & & 0.78 & 2.50 & & & 366 & 739 & 816 \\
\hline LSTM & 0.77 & 0.94 & 1.21 & 90.0 & 39.1 & 131 & 80.9 & 183 \\
\hline TB & 0.55 & 0.71 & 3.02 & 677 & 631 & 824 & 805 & 1539 \\
\hline $\begin{array}{l}\mathrm{N}- \\
\text { Ordered } \\
(n= \\
1000)\end{array}$ & 0.21 & 0.13 & 7.08 & 1893 & 2920 & 2103 & 4132 & 5155 \\
\hline
\end{tabular}

Table 5.10: The results of imitating the Switch expert.

happen more often with TB than the MLP. This is likely due to TB comparing past environment states with a bias towards more recent environment states, making it more likely to get stuck alternating its turn direction.

The n-ordered similarity metric failed to imitate both the reactive and state-based components of the Switch expert due to how large $n$ is. It consistently got stuck performing one action regardless of the current environment state.

Our results in Table 5.10 support what we observed, with the LSTM performing better than all other techniques, and the MLP and TB performing similarly. Our proposed performance metrics also supported the observed disparity we mentioned between the performance of the MLP and the TB agents. Our results also confirmed that the n-ordered similarity metric was the worst performing agent.

\subsubsection{Offense Expert Results}

In Table 5.11, we present the results of our experiment with the Offense expert. Our observations on the behavior of the agents indicated that the LSTM was the closest 


\begin{tabular}{lllllllll}
\hline \multicolumn{7}{c}{ Monte } \\
Agent & Macro & Weighted Carlo \\
& F1 & F1 & $\begin{array}{c}\text { Dis- } \\
\text { tance }\end{array}$ & $\chi_{P(S)}^{2}$ & $\chi_{P(A)}^{2}$ & $\chi_{P\left(S_{t}, S_{t-1}\right)}^{2} \chi_{P\left(A_{t}, A_{t-1} \chi_{P(A, S)}^{2}\right.}^{2}$ \\
\hline MLP & 0.70 & 0.79 & 2.12 & 42.7 & 136 & 155 & 396 & 436 \\
LSTM & 0.70 & 0.79 & 2.26 & 135 & 12.2 & 173 & 84.2 & 505 \\
TB & 0.68 & 0.75 & 2.08 & 67.4 & 23.3 & 97.4 & 41.5 & 551 \\
N- & & & & & & & & \\
Ordered \\
$\begin{array}{l}(n= \\
80)\end{array}$
\end{tabular}

Table 5.11: The results of imitating the Offense expert.

agent to the behavior of the Offense expert. The second closest agent to the behavior of the Offense expert was TB. While both the TB and LSTM agents always dashed till they lost sight of the ball before they started turning to the ball, the TB agent was worse at properly identifying the situations it should kick the ball, and the situations where it should look for the ball. Again, the n-ordered similarity metric was nowhere close to the behavior of the expert. Lastly, the MLP agent dashed most of the time even in cases where it should turn to the ball.

Our results in Table 5.11 disagree on most of what we observed when we use the macro and weighted F1-score, and the Monte Carlo distance. However, the Offense expert has a behavior that exhibits a characteristic $P\left(A_{t}, A_{t-1}\right)$ distribution, due to the ratio of times it dashes consecutively to the times it turns or kicks after a dash. If an agent performs the correct actions in most scenarios for this expert, we expect that its $P\left(A_{t}, A_{t-1}\right)$ to be small. In Table 5.11, we see that the LSTM agent is closer to the $P\left(A_{t}, A_{t-1}\right)$ than the MLP which is inline with what we observed. TB suffered from imitating the reactive parts of the Offense expert which is apparent in the difference 
between the F1-scores. The MLP agent has a higher F1-score than the TB agent but has a much worse $\chi_{P\left(A_{t}, A_{t-1}\right)}^{2}$ which is inline with what we observed: TB is worse at imitating the reactive component of the Offense expert, but better at imitating the state-based component.

We can conclude form this experiment that it is important to analyze the distribution that is characteristic to the expert imitated, in addition to the F1-scores, to determine which agent is qualitatively better at imitating the expert. We can not conclude that the LSTM was the best technique at imitating the Offense expert because TB may be able to outperform it if it used a larger training set. However, we can conclude that the LSTM is the better technique at imitating the Offense expert with a high run-time responsiveness.

\subsubsection{Pro Expert Results}

\begin{tabular}{lllllllll}
\hline \multicolumn{7}{c}{ Monte } \\
Agent & Macro & Weighted Carlo \\
& F1 & F1 & $\begin{array}{c}\text { Dis- } \\
\text { tance }\end{array}$ & $\chi_{P(S)}^{2}$ & $\chi_{P(A)}^{2}$ & $\chi_{P\left(S_{t}, S_{t-1}\right)}^{2} \chi_{P\left(A_{t}, A_{t-1}\right)}^{2} \chi_{P(A, S)}^{2}$ \\
\hline MLP & 0.79 & 0.93 & 1.33 & 92.6 & 109 & 127 & 197 & 226 \\
LSTM & 0.81 & 0.96 & 1.11 & 13.8 & 32.0 & 38.5 & 60.6 & 142 \\
TB & 0.44 & 0.71 & 2.82 & 280 & 20.5 & 356 & 85.3 & 1126 \\
N- & & & & & & & & \\
$\begin{array}{l}\text { Ordered } \\
(n=\end{array}$ & 0.07 & 0.08 & 7.52 & 2548 & 3295 & 2819 & 4494 & 6111 \\
$10)$ & & & & & & & & \\
\hline
\end{tabular}

Table 5.12: The results of imitating the Pro expert.

The results of imitating the Pro expert are presented in Table 5.12. LSTM was better at imitating this expert compared to any other technique. However, we found 
that MLP was better than TB at imitating the Pro expert. This is likely due to the slight difference between the environment state vector of the MLP agent and the TB agent. Since TB is a CBR technique, it does not need the visibility features because missing features are penalized by the similarity metric. However, this also meant that it will not have the last visible direction of the ball in its most recent environment state vector unlike the MLP agent. This resulted in the MLP correctly turning in the direction of the ball most of the time compared to TB: which requires to backtrack to find the last direction of the ball.

The difference between the LSTM agent's performance and the MLP agent lies in how quickly they react to a missing ball. While LSTM can notice the change of the ball visibility feature from a 1 to a 0 between two time steps; the MLP agent can only react to the current environment state vector. Hence, the MLP agent may get "stuck" in a situation where it is repeatedly performing an action such as kick when it loses sight of the ball. On the other hand, the LSTM's hidden state will, eventually, if not immediately, change to signify that the ball is missing, resulting in the LSTM not getting stuck in scenarios where the MLP got stuck.

The n-ordered similarity metric was again nowhere close to the behavior of the expert and failed to imitate both its reactive and state-based components.

Our results in Table 5.12 are in line with what we observed from our agents. Again, n-ordered similarity was the worst performing technique, since this expert uses the most recent environment state when deciding which action to take majority of the time.

Therefore, we can conclude from this experiment that the LSTM agent performed best at imitating the Pro expert. We can also conclude that the environment state vector can make state-based behavior that requires recalling a particular feature, 
feasible for imitation learning techniques that can only learn reactive behavior.

\subsubsection{Goal Expert Results}

\begin{tabular}{lllllllll}
\hline \multicolumn{7}{c}{ Agent } & Macro & Weighted Carlo \\
& F1 & F1 & $\begin{array}{c}\text { Dis- } \\
\text { tance }\end{array}$ & $\chi_{P(S)}^{2}$ & $\chi_{P(A)}^{2}$ & $\chi_{P\left(S_{t}, S_{t-1}\right)}^{2} \chi_{P\left(A_{t}, A_{t-1}\right)}^{2} \chi_{P(A, S)}^{2}$ \\
\hline MLP & 0.82 & 0.94 & 1.36 & 40.9 & 96.8 & 87.1 & 148 & 304 \\
LSTM & 0.93 & 0.98 & 0.93 & 3.14 & 2.77 & 14.8 & 15.2 & 66.5 \\
TB & 0.50 & 0.59 & 3.88 & 207 & 261 & 349 & 433 & 1889 \\
N- & & & & & & & & \\
$\begin{array}{l}\text { Ordered } \\
(n=\end{array}$ & 0.08 & 0.03 & 7.62 & 2423 & 3801 & 2779 & 5393 & 5958 \\
$400)$ & & & & & & & & \\
\hline
\end{tabular}

Table 5.13: The results of imitating the Goal expert.

In the case of imitating the goal expert, LSTM again outperformed all other techniques. It correctly turned in the direction of the goal when it is looking for the goal. Similar to the Pro expert, the second best technique at imitating this expert was the MLP due to the way we modelled the environment state input of RoboCup soccer. The difference between MLP and LSTM is that the MLP agent sometimes got stuck attempting to kick the ball when it could not see the goal and in some cases, it got stuck attempting to kick the ball when it cannot see the ball. This is for the same reason as in the previous Pro expert experiment: the LSTM agent will eventually correct its action since it will notice the change of the visibility feature from 1 to 0 over a few time steps if not immediately. Again, the n-ordered similarity metric was the worst technique at imitating the Goal expert. The results in Table 5.13 support what we observed. 
Similar to the Pro expert, we can conclude that the LSTM agent was the best agent at imitating the Goal expert. Our results also further support our conclusion of the Pro expert: carefully modelling the input of an expert may enable techniques that previously couldn't imitate state-based behavior to be able to do so. This is particularly true for state-based experts that need to recall a feature of a previously visible object, such as the Pro and Goal experts.

\subsubsection{Pro Goal Expert Results}

\begin{tabular}{|c|c|c|c|c|c|c|c|c|}
\hline \multirow{3}{*}{$\begin{array}{l}\text { Agent } \\
\text { MLP }\end{array}$} & \multirow{3}{*}{$\begin{array}{l}\text { Macro } \\
\text { F1 } \\
0.55\end{array}$} & \multirow{2}{*}{\multicolumn{2}{|c|}{$\begin{array}{c}\text { Monte } \\
\text { d Carlo } \\
\text { Dis- } \\
\text { tance }\end{array}$}} & \multirow{3}{*}{$\begin{array}{l}\chi_{P(S)}^{2} \\
372\end{array}$} & \multirow{3}{*}{$\begin{array}{l}\chi_{P(A)}^{2} \\
1038\end{array}$} & \multirow{2}{*}{\multicolumn{3}{|c|}{$\chi_{P\left(S_{t}, S_{t-1}\right)}^{2} \chi_{P\left(A_{t}, A_{t-1}\right)}^{2} \chi_{P(A, S)}^{2}$}} \\
\hline & & & & & & & & \\
\hline & & 0.51 & 4.98 & & & 602 & 1707 & 2644 \\
\hline LSTM & 0.87 & 0.97 & 1.14 & 62.3 & 81.3 & 113 & 126 & 169 \\
\hline $\mathrm{TB}$ & 0.59 & 0.73 & 2.79 & 314 & 239 & 422 & 388 & 1267 \\
\hline $\begin{array}{l}\text { N- } \\
\text { Ordered } \\
(n= \\
400)\end{array}$ & 0.04 & 0.03 & 7.68 & 2632 & 3897 & 2903 & 5296 & 6248 \\
\hline
\end{tabular}

Table 5.14: The results of imitating the Pro Goal expert.

The results of this experiment are the most interesting out of all of our RoboCup experts. The LSTM agent was much better at imitating the Pro Goal expert than all other agents. This time, TB was closer to the behavior of the Pro Goal expert than MLP and this was also supported by our results in Table 5.14. Consistent with the pattern we noticed in all our experiments with state-based experts, the n-ordered agent was no where close to the behavior of the Pro Goal expert.

While MLP was able to perform close to LSTM in the Pro expert and the Goal 
expert, it was unable to competently imitate the combined behaviors in the Pro Goal expert. The MLP agent correctly identified which direction to turn in when it lost sight of the ball/goal; however, it was so much worse at imitating the expert's reactive behavior such as dashing to the ball. It often got stuck turning to the ball instead of dashing to the ball and often attempted to kick the ball before it was close enough, even with a large training set of 64165 cases that is more than twice the size of the training set of the LSTM and TB agents combined. Moreover, all the features of the current environment state vector should allow the MLP agent to imitate this behavior, as it did with the Pro and Goal experts.

\subsubsection{Conclusion}

In this section, we compared our proposed imitation learning techniques to TB and n-ordered similarity. We found that the LSTM agent performed the best compared to all other techniques at imitating all of the state-based experts we designed in the RoboCup soccer domain. However, the MLP agent imitated the reactive expert better than all other agents; while the LSTM agent was a close second. We also saw more examples of how our proposed performance metrics give us insight that other metrics do not, such as our proposed $\chi_{P\left(A_{t}, A_{t-1}\right)}^{2}$ metric in the case of the Offense expert. This example also showed the importance of using the distribution that is characteristic to the state-based expert imitated, when comparing which technique performs better at imitating the expert.

We also saw how the environment state input can result in reactive imitation learning techniques such as MLP being able to imitate state-based experts (in the case of the Pro and Goal experts). However, we found that MLP was not able to properly imitate the Pro Goal expert despite having access to all the features it needs 
to imitate it. The MLP was significantly worse at identifying when to dash to the ball, and often attempted to kick or turn to the ball instead of dash, when imitating the Pro Goal expert. In the next section, we will present and discuss our experiments in the vacuum cleaner domain.

\subsection{Vacuum Cleaner Experiments}

\subsubsection{Introduction}

We divided this section into two parts that each pertain to an experiment we performed. The first part compares the results obtained using our testing methodology to those obtained using the methodology adopted by Gunaratne et al. [4] and Ontañón et al. [1] The objective of this experiment is to show that online testing is more reliable than offline testing by evaluating the consistency of the results obtained by each testing methodology with the qualitative performance of the agents. The second part of this section compares the performance of our proposed imitation learning techniques to the state-of-the-art techniques in the vacuum cleaner domain using online testing. In this experiment, we show how no performance metric is self-sufficient at telling us which agent imitated the expert better. We also show how the performance metrics, that we select using prior knowledge about the expert from the metrics we propose, provide results that are consistent with the qualitative performance of the agents. Moreover, we will observe that LSTM may not perform as well as other state-of-the-art techniques in the discrete domain. 


\subsubsection{An Experiment of Testing Methodologies}

Recall from Section 3.4, that there are two types of testing: online testing and offline testing. Online testing is where the trained agent is tested by deploying it in the environment and producing the agent's own trajectory. In contrast, offline testing is where the agent is used to predict the action it should take on a pre-existing trajectory of the expert. Gunaratne et al. [4] and Ontañón et al. [1] obtained their results by employing offline testing. They compared the predicted outputs of their agents to the actual outputs of the expert on a trajectory of the expert. We discussed how this does not provide results that correspond with the qualitative performance of the agents in the real environment, due to the actions (outputs) not affecting the trajectory of their agents during testing. In the case where the agents are running in the environment, their outputs affect the next environment state. Hence, their trajectories and future actions will be different. This may also result in cases where their agents are stuck in an environment state due to repeatedly executing an invalid action at that environment state.

On the other hand, we employed an online testing methodology in this thesis, which we hypothesized to provide results that resemble the qualitative performance of our agents. As we mentioned in Section 3.4, online testing involves running the trained agents in the real environment and either using a pre-existing trajectory of the expert to compare the trajectory of the agent and the expert it is imitating, or using the expert's feedback during run-time to obtain predictive accuracy and F1 scores.

In this experiment, we reproduced the results of Gunaratne et al. [4] using their offline testing methodology and compared them to the results we obtained using our online testing methodology. The goal of this experiment is to test our hypothesis that the online testing method provides accurate results that correlate with the performance 
of the agents at imitating the experts in the environment, while offline testing does not.

\subsubsection{Experiment Setup}

Recall from Section 3.2.2.2, there are 5 experts that were used by Ontañón et al. [1] in the vacuum cleaner domain: the Smart Random, Smart Straight Line, ZigZag, Fixed Sequence, and Smart Explorer experts. However, we will only use the experts that were used in both the work of Ontañón et al. [1] and Gunaratne et al. [4] as we are replicating the hyper-parameters of Gunaratne et al. [4] This leaves us with 4 experts: the Smart Random, Smart Straight Line, ZigZag, and Fixed Sequence experts.

The Smart Random expert selects an action at random (either up, down, left, right, or stand still); however, if it sees dirt in only one direction it will move in that direction. This is a reactive and stochastic agent since it randomly chooses the action it takes and when it selects an action deterministically, it selects it based on the current environment state.

The Smart Straight Line expert selects a direction at random and then moves in a straight line until it collides with a wall where it will repeat this behavior. Again, if it sees dirt in only one direction, it will choose that direction.

The ZigZag expert moves in a zig-zag pattern: it moves right until it collides, at this point it moves down once and then repeats this behavior moving to the left. Once it can no longer go down, it repeats the same behavior going upwards instead.

The Fixed Sequence expert repeats the same fixed sequence of actions that is 21 actions long.

We will use those experts in our experiment to compare the testing methodology of Gunaratne et al. [4] to our testing methodology. 
Agents We used LfODBN and TB in this experiment with the same training methodology and hyper-parameters used in the work of Gunaratne et al. [4]. The problem and solution threshold of TB were both set to 0.99, and the LfODBN was trained for 20 iterations of EM.

Testing Methodology of Gunaratne et al. [4] The testing methodology of Gunaratne et al. [4] involved using 10 datasets per map. There are 7 maps: five 8x8 maps and two 32x32 maps. Those maps can be found in the work of Ontañón et al. [1]. Each dataset consisted of 1000 entries. Since there are 70 datasets per expert, 70-fold-cross validation was used to obtain the macro F1 score. The macro F1-score was calculated by comparing the predicted actions of the trained agent to the actions of the expert in the left out dataset.

Online Testing Methodology For our online testing methodology, we opted to use one dataset per map, excluding the $7^{\text {th }}$ map (i.e. the $32 \times 32$ map), as our training set. Each dataset consists of 1000 entries; therefore, the training set of each agent consisted of 6000 entries. We also did not perform cross validation; thus, each agent was trained on datasets obtained from maps 1 to 6 and was tested by running it in the $7^{\text {th }}$ map. The macro F1 score was calculated by comparing the actions the trained agent took during its run to the actions suggested by the expert. We also computed our proposed metrics, that compare a run of the trained agent to a run of the expert, to see if they provide us with results that correlate with what we observed. We chose the testing map as the $7^{\text {th }}$ map because it is the largest map. 


\begin{tabular}{llllllll}
\hline \multirow{2}{*}{ Agent } & $\begin{array}{l}\text { Offline } \\
\text { Macro }\end{array}$ & $\begin{array}{l}\text { Online } \\
\text { Macro } \\
\text { F1 }\end{array}$ & $\begin{array}{l}\text { Monte } \\
\text { Carlo } \\
\text { Dis- } \\
\text { tance }\end{array}$ & $\chi_{P(S)}^{2}$ & $\chi_{P(A)}^{2}$ & $\chi_{P\left(S_{t}, S_{t-1}\right)}^{2} \chi_{P\left(A_{t}, A_{t-1}\right)}^{2}$ \\
\hline \multirow{2}{*}{ TB } & 0.34 & 0.10 & 4.36 & 805 & 635 & 1940 & 1445 \\
LfODBN & 0.27 & 0.22 & 2.74 & 41.8 & 6.06 & 73.9 & 17.6 \\
\hline
\end{tabular}

Table 5.15: A comparison between the F1 scores obtained using the methodology of Gunaratne et al. [4] (the offline macro F1) and the results obtained using our methodology when imitating the Smart Random expert.

\subsubsection{Results}

Smart Random Expert The results of imitating the Smart Random expert are presented in Table 5.15. From observing the two agents run in the testing map, the TB agent got stuck alternating between the down and up actions 19 time steps into the map, whereas the LfODBN agent was randomly selecting the action and selecting the direction that has dirt in the correct scenarios. It was clear from watching the two agents that LfODBN outperformed TB.

From our results in Table 5.15, we can see that the F1 score obtained using our method (the online macro F1 score) is very different to the one obtained using the method of Gunaratne et al. [4]. Moreover, all our proposed metrics including the macro F1 score obtained using our testing method agree that LfODBN outperformed TB at imitating the Smart Random expert; whereas, the macro F1 score obtained using the methodology of Gunaratne et al. [4] show that TB outperformed LfODBN. This is an example where our online testing methodology matched what we observed and the offline testing methodology did not.

In addition, our $\chi_{P(A)}^{2}$ metric makes the most sense to use here compared to other 
metrics. This is because the Smart Random expert selects its action randomly the majority of the time. Thus, if the distribution of actions selected by the agent is close to the distribution of actions selected by the expert, then the agent successfully learnt the stochastic behavior of the expert.

\begin{tabular}{llllllll}
\hline \multirow{2}{*}{ Agent } & $\begin{array}{l}\text { Offline } \\
\text { Macro }\end{array}$ & $\begin{array}{l}\text { Online } \\
\text { Macro } \\
\text { F1 }\end{array}$ & $\begin{array}{l}\text { Monte } \\
\text { Carlo } \\
\text { Dis- } \\
\text { tance }\end{array}$ & $\chi_{P(S)}^{2}$ & $\chi_{P(A)}^{2}$ & $\chi_{P\left(S_{t}, S_{t-1}\right)}^{2}$ & $\chi_{P\left(A_{t}, A_{t-1}\right)}^{2}$ \\
\hline \multirow{2}{*}{ TB } & 0.93 & 0.24 & 3.70 & 1382 & 401 & 1440 & 419 \\
LfODBN & 0.74 & 0.34 & 3.02 & 152 & 36.9 & 176 & 73.2 \\
\hline
\end{tabular}

Table 5.16: A comparison between the F1 scores obtained using the methodology of Gunaratne et al. [4] (the offline macro F1) and the results obtained using our methodology when imitating the Smart Straight Line expert.

Smart Straight Line Expert We observed the LfODBN agent imitate the Smart Straight Line expert very well. It selects a random valid direction once it reaches an obstacle and when it finds dirt in one direction, it switched its path towards that direction. The low online macro F1 score is due to the stochastic component of the expert: sometimes the expert will choose a random direction that is different to that chosen by the LfODBN agent. On the other hand, the TB agent was also able to replicate the behavior of the Smart Straight Line expert; however, it did not move in the direction of dirt when there was dirt in one direction. Thus, we can say that it failed to imitate the "smart" aspect of the Straight Line expert. Therefore, we can safely say that the LfODBN agent imitates the Smart Straight Line expert better than the TB agent.

Our results in Table 5.16, show that all the metrics we obtained using our online 
testing methodology are in agreement with what we observed: the LfODBN imitates the Smart Straight Line expert better than the TB agent. However, the macro F1 score obtained using offline testing (i.e. the methodology used by Gunaratne et al. [4]) says the contrary: the LfODBN imitated the Smart Straight Line expert worse than the TB agent. This is another example showing the inadequacy of obtaining results using offline testing and that we should use online testing to obtain results.

\begin{tabular}{llllllll}
\hline \multirow{2}{*}{ Agent } & $\begin{array}{l}\text { Offline } \\
\text { Macro }\end{array}$ & $\begin{array}{l}\text { Online } \\
\text { Macro }\end{array}$ & $\begin{array}{l}\text { Monte } \\
\text { Carlo } \\
\text { F1 }\end{array}$ & $\begin{array}{l}\text { Fis- } \\
\text { tance }\end{array}$ & $\chi_{P(S)}^{2}$ & $\chi_{P(A)}^{2}$ & $\chi_{P\left(S_{t}, S_{t-1}\right)}^{2} \chi_{P\left(A_{t}, A_{t-1}\right)}^{2}$ \\
\hline & & & & & & & \\
TB & 0.87 & 0.80 & 3.05 & 501 & 23.9 & 662 & 105 \\
LfODBN & 0.64 & 0.48 & 2.73 & 217 & 45.1 & 369 & 95.2 \\
\hline
\end{tabular}

Table 5.17: A comparison between the F1 scores obtained using the methodology of Gunaratne et al. [4] (the offline macro F1) and the results obtained using our methodology when imitating the ZigZag expert.

ZigZag Expert In this experiment, we observed the LfODBN somewhat imitating the ZigZag expert adequately. It often stood still instead of moving in a direction once it reached an obstacle; however, it moved in the correct direction on the next step. Moreover, it would sometimes turn left then right, which is something the expert never does. It also went up and down randomly instead of properly performing the zig-zag pattern till it reached a corner. On the other hand, the TB agent was able to imitate the ZigZag expert much better. The TB agent never stood still. However, once it reached the bottom corner, it got stuck alternating its zig-zag direction between the up and down directions.

Our results in Table 5.17 show that the TB agent outperformed the LfODBN agent 
in both the offline and online macro F1 scores. However, we saw how unreliable offline testing can be in the previous two experts and thus it does not provide consistent results all the time. In this experiment, $\chi_{P(S)}^{2}$ and $\chi_{P\left(S_{t}, S_{t-1}\right)}^{2}$ seem to not agree with what we observed. This is because the TB agent got stuck in a circle where it saw the bottom corners often in comparison to the LfODBN. Hence, we can not use a single $\chi^{2}$ metric to evaluate how well our agents performed. We need to look at all metrics including the $\chi_{P(A)}^{2}$ metric, that shows the disparity between the action distribution of the LfODBN and the ZigZag expert: the LfODBN performs the stand still action which the expert never performs.

\begin{tabular}{llllllll}
\hline \multirow{2}{*}{ Agent } & $\begin{array}{l}\text { Offline } \\
\text { Macro }\end{array}$ & $\begin{array}{l}\text { Online } \\
\text { Macro } \\
\text { F1 }\end{array}$ & $\begin{array}{l}\text { Monte } \\
\text { Carlo } \\
\text { Dis- } \\
\text { tance }\end{array}$ & $\chi_{P(S)}^{2}$ & $\chi_{P(A)}^{2}$ & $\chi_{P\left(S_{t}, S_{t-1}\right)}^{2} \chi_{P\left(A_{t}, A_{t-1}\right)}^{2}$ \\
\hline \multirow{2}{*}{ TB } & 0.67 & 0.1 & 6.92 & 667 & 1333 & 1075 & 1998 \\
LfODBN & 0.42 & 0.18 & 6.19 & 926 & 106 & 1067 & 918 \\
\hline
\end{tabular}

Table 5.18: A comparison between the F1 scores obtained using the methodology of Gunaratne et al. [4] (the offline macro F1) and the results obtained using our methodology when imitating the Fixed Sequence expert.

Fixed Sequence Expert Here, neither the LfODBN agent nor the TB agent were able to imitate the Fixed Sequence expert adequately. However, it was clear that the LfODBN was closer to the behavior of the expert than TB. TB performed the left action during its entire 1000 steps in the testing map and never changed the action. On the other hand, the LfODBN agent switched the actions it is performing to match some sub-sequences of the sequence the expert performs; however, it still did not match the sequence majority of the time. 
The results in Table 5.18 highlight the unreliability of offline testing again, similar to the previous experts. Here, the offline macro F1 score shows that the TB agent outperformed the LfODBN, contrary to what we observed. On the other hand, the online F1 score, $\chi_{P(A)}^{2}$, and $\chi_{P\left(A_{t}, A_{t-1}\right)}^{2}$ that we obtained using our online testing methodology were in line with what we observed: the LfODBN has better results than the TB agent. In this scenario, the $\chi_{P(S)}^{2}$ and $\chi_{P\left(S_{t}, S_{t-1}\right)}^{2}$ are not very meaningful since the Fixed Sequence expert performs a sequence of actions without regard to the environment. Therefore, we are trying to match the action trajectory of the agent with the action trajectory of the expert rather than the environment state trajectory.

\subsubsection{Conclusion}

In this experiment, we showed how the offline testing methodology that is adopted by many researchers in the field of imitation learning is inadequate at reflecting the performance of an imitation learning agent at imitating a particular expert. We also showed how our testing methodology that makes use of online testing provides results that are consistent with the qualitative performance of the vacuum cleaner agents in the testing map. Hence, we employed our testing methodology when comparing our proposed imitation learning techniques to state-of-the-art techniques. This experiment validated our online testing methodology and showed that the offline testing methodology does not provide reliable results when comparing imitation learning techniques. 


\subsubsection{Imitation Learning Performance of LSTM and MLP}

\subsubsection{Introduction}

In this experiment, we compare the performance of MLP and LSTM to state-of-the-art imitation learning techniques at imitating the experts we used in the previous vacuum cleaner experiment (refer to Section 5.5.2.1). We used the online testing methodology we described in Section 5.5.2.1 as we showed how it provides results consistent with the performance of the agents. We hypothesize that the LSTM network will be able to imitate the ZigZag expert better than other techniques. However, we hypothesize that the LSTM network will under-perform in comparison to the other techniques when imitating the Smart Straight Line expert, and the Fixed Sequence expert. This is because the LSTM does not use its previous action to compute its hidden internal state.

\subsubsection{Experiment Setup}

The setup of this experiment is almost identical to the setup of the previous experiment; however, we only use the online testing methodology we described in Section 5.5.2.1 since it is much more reliable than offline testing. We also used the Smart Random, Smart Straight Line, ZigZag, and Fixed Sequence experts that we used in the previous experiment. To learn more about the online testing methodology and the experts of this experiment refer to the relevant paragraphs in Section 5.5.2.1.

Agents In addition to the LfODBN and the TB agents, we used the n-ordered agent with $n=11$. We selected $n=11$ as this was the hyper-parameter used by Gunaratne et al. [2] in his experiment in the vacuum cleaner domain. We used the same LSTM and MLP used in the RoboCup soccer domain. 
The LSTM neural network was trained using a dataset obtained from 6 vacuum cleaner maps (the same maps as the previous experiment), that contain 1000 environment state and action pairs each. The LSTM was trained using the same method we mentioned in Section 4.3.1.2: 1000 epochs of training using Adam where the entire dataset (6000 entries) is considered as one sequence. The learning step of the LSTM was set to $\lambda=0.001$.

Similarly, the MLP neural network was trained for 1000 epochs over the dataset obtained from the same 6 vacuum cleaner maps with the learning step set to $\lambda=0.001$. We used the same datasets that we collected in Section 5.5.2 to train both the MLP and LSTM agents.

\subsubsection{Results}

\begin{tabular}{lllllllll}
\hline \multicolumn{7}{c}{ Monte } \\
Agent & Macro & Weighted Carlo & $\chi_{P(S)}^{2}$ & $\chi_{P(A)}^{2}$ & $\chi_{P\left(S_{t}, S_{t-1}\right)}^{2} \chi_{P\left(A_{t}, A_{t-1}\right)}^{2} \chi_{P(A, S)}^{2}$ \\
& F1 & F1 & $\begin{array}{l}\text { Dis- } \\
\text { tance }\end{array}$ & & & & & \\
\hline MLP & 0.20 & 0.16 & 2.92 & 631 & 692 & 1826 & 1548 & 1493 \\
LSTM & 0.31 & 0.66 & 5.84 & 1872 & 507 & 1897 & 1360 & 1880 \\
TB & 0.10 & 0.05 & 4.36 & 805 & 635 & 1940 & 1445 & 1443 \\
N- & & & & & & & & \\
Ordered & 0.19 & 0.18 & 3.79 & 701 & 164 & 922 & 489 & 896 \\
$\begin{array}{l}(n= \\
11)\end{array}$ & & & & & & & & \\
LfODBN 0.22 & 0.27 & 2.74 & 41.8 & 6.06 & 73.9 & 17.6 & 66.7 \\
\hline
\end{tabular}

Table 5.19: The results of imitating the Smart Random expert.

Smart Random Expert The MLP agent got stuck at the start of its trajectory and kept alternating between the right and left direction. This is expected because 
the MLP will always select the same action for a particular environment state, as it basis the action it takes on the current environment state alone.

The LSTM agent got stuck alternating between going up and down when there was a tile of dirt 2 tiles above it. It went up towards the dirt tile; however, when it saw that the dirt tile is adjacent to it, it went down instead of going up and kept repeating this till the end of its 1000 steps. Before it got stuck, it seemed to select the same action for many consecutive time steps before it changed the action it took. The reason the macro F1 score of the LSTM agent is high is because it should have moved up in all the situations it was stuck in; which made up the majority of its trajectory. In fact, an expert that adequately imitates the Smart Random expert should have a low F1 score because the expert selects its actions at random majority of the time.

The TB agent also got stuck as we mentioned in the previous experiment, alternating between the down and up actions instead of randomly selecting its action.

The n-ordered agent seemed to select its actions at random all of the time, even when there was a dirt tile in one of its direction. While the n-ordered agent is able to replicate the stochastic behavior of the Smart Random expert, it does not replicate its deterministic behavior: i.e. moving in the direction of the dirt when one direction contains dirt.

The LfODBN managed to outperform all techniques in this experiment as we saw in the previous experiment: it turned towards the direction of the dirt when there was dirt in one direction and seemed to select the direction at random when there is no dirt in any direction.

The best performance metric to use to compare the performance of the agents in this experiment would be the $\chi_{P\left(A_{t}, A_{t-1}\right)}^{2}$. This is because the Smart Random expert randomly chooses its direction except in the case where there is dirt in one direction, 
where it will repeatedly select that direction till it removes the dirt. When we compare the agents using this metric in Table 5.19, we see how the LfODBN performed the best, with the n-ordered agent being the second best as we observed. We can also see how the rest of the techniques did not perform well at imitating the Smart Random expert.

\begin{tabular}{lllllllll}
\hline \multicolumn{7}{c}{ Monte } \\
Agent & Macro & Weighted Carlo & & & & \\
F1 & F1 & $\begin{array}{c}\text { Dis- } \\
\text { tance }\end{array}$ & $\chi_{P(S)}^{2}$ & $\chi_{P(A)}^{2}$ & $\chi_{P\left(S_{t}, S_{t-1}\right)}^{2} \chi_{P\left(A_{t}, A_{t-1}\right)}^{2} \chi_{P(A, S)}^{2}$ \\
\hline MLP & 0.22 & 0.66 & 4.44 & 1562 & 878 & 1931 & 1918 & 1757 \\
LSTM & 0.24 & 0.09 & 2.94 & 1235 & 723 & 1281 & 741 & 1278 \\
TB & 0.24 & 0.15 & 3.70 & 1382 & 401 & 1440 & 419 & 1446 \\
N- & & & & & & & & \\
Ordered & 0.19 & 0.38 & 2.74 & 700 & 1.87 & 776 & 42.2 & 726 \\
$\begin{array}{l}(n= \\
11)\end{array}$ & & & & & & & & \\
LfODBN 0.34 & 0.48 & 3.02 & 152 & 36.9 & 176 & 73.2 & 172 \\
\hline
\end{tabular}

Table 5.20: The results of imitating the Smart Straight Line expert.

Smart Straight Line Expert The MLP agent started alternating its move direction between left and right 16 time steps into the trajectory after removing a dirt pile in the south direction. This is expected, as the MLP can not imitate the state-based behavior of the Smart Straight Line expert. The agent needs to remember the previous action it took in order to imitate the Smart Straight Line expert; however, the MLP only uses the current environment state when deciding which direction to move in. Its high weighted F1-score is due to the expert choosing the right direction when it was stuck alternating between the left and right directions.

The LSTM agent seemed to imitate the Smart Straight Line expert quite well. It 
moved in the direction of dirt when there was dirt in one direction. It also selected a random direction and kept moving in that direction when there was no dirt in any direction. However, it occasionally changed the direction it moved in when more directions were available, instead of changing it when it reached an obstacle.

As we mentioned in the previous experiment, the TB agent seemed to imitate the Smart Straight Line expert well. It continually moved in one direction till it reached an obstacle, where it then switches its move direction. However, in many situations where there was one direction that contained dirt, it did not change its direction.

The n-ordered agent performed very similarly to the TB agent. However, it never changed its direction to the direction of the dirt when there was dirt in one direction. Instead, it continued moving in the direction it was moving till it reached an obstacle, where it randomly selected a new direction.

Again, as we mentioned in the previous experiment, the LfODBN agent outperformed all other techniques. It did not make the mistakes that other techniques made and was almost identical to the expert's behavior when we observed it.

It is difficult to find a performance metric that suites this behavior because its actions are stochastic, yet repeated for a long time. This will result in $\chi_{P(A)}^{2}$ and $\chi_{P\left(A_{t}, A_{t-1}\right)}^{2}$ not being reliable performance metrics in the case where the agent/expert selected a different random direction. However, the performance metric that should provide us with most information when evaluating agents at imitating this expert is the $\chi_{P\left(S_{t}, S_{t-1}\right)}^{2}$. This is because most of the environment states that the agent sees as it is moving in one direction should be the same till it reaches an obstacle or till it finds a dirt in a different direction. At the environment state where it finds dirt, it will switch its turn direction and should quickly get rid of environment states that contain dirt tiles. Looking at the results in Table 5.20, we see that the $\chi_{P\left(S_{t}, S_{t-1}\right)}^{2}$ performance 
metric is in line with what we observed: the LfODBN did the best, followed by the TB agent, then the LSTM agent, and lastly, the n-ordered agent.

\begin{tabular}{lllllllll}
\hline \multicolumn{7}{c}{ Agent } & Macro & \multicolumn{3}{c}{ Weighted Carlo } \\
& F1 & F1 & $\begin{array}{c}\text { Dis- } \\
\text { tance }\end{array}$ & $\chi_{P(S)}^{2}$ & $\chi_{P(A)}^{2}$ & $\chi_{P\left(S_{t}, S_{t-1}\right)}^{2} \chi_{P\left(A_{t}, A_{t-1}\right)}^{2} \chi_{P(A, S)}^{2}$ \\
\hline MLP & 0.22 & 0.67 & 2.47 & 724 & 99.2 & 1722 & 1986 & 1189 \\
LSTM & 0.65 & 0.95 & 2.46 & 356 & 3.13 & 540 & 121 & 479 \\
TB & 0.80 & 0.96 & 3.05 & 501 & 23.9 & 662 & 105 & 880 \\
N- & & & & & & & & \\
$\begin{array}{l}\text { Ordered } \\
(n=\end{array}$ & 0.19 & 0.02 & 6.94 & 1687 & 724 & 1838 & 812 & 1935 \\
$\begin{array}{l}\text { 11 }) \\
\text { LfODBN } 0.48\end{array}$ & 0.85 & 2.73 & 217 & 45.1 & 369 & 95.2 & 241 \\
\hline
\end{tabular}

Table 5.21: The results of imitating the ZigZag expert.

ZigZag Expert The MLP agent failed to imitate the ZigZag expert. This was expected, as the ZigZag expert experiences state-based behavior and the MLP is not capable of imitating state-based behavior. It was stuck alternating its move direction between right and left throughout its 1000 step trajectory in the testing map.

On the other hand, the LSTM agent was able to imitate the ZigZag expert somewhat adequately. It correctly moved down initially then continued moving in the right direction. However, when it reached the right side it did not move in the down direction. Instead it moved in the up direction as if it reached a bottom corner. It was not always consistent in the vertical direction it moved in, and eventually, it got stuck doing a circle that spans the obstacles to its right and left sides before reaching the bottom corner.

The TB agent was able to imitate the ZigZag expert very similarly to the LSTM 
agent. However, it made mistakes that LSTM never makes, such as moving in the same (right/left) direction that it was moving in after moving downwards once or upwards once. It also got stuck moving in a circle one it reached the bottom corner of the map. This was the technique that reached the furthest environment state that the ZigZag expert visited (i.e. the bottom corner).

The n-ordered expert started off well, but eventually, it was nowhere close to the behavior of the ZigZag expert. Its reactions to reaching obstacles to its right and left are very delayed, it started by being 1 time step delayed, then 2 and eventually it kept trying to move left when it already reached an obstacle till the end of its trajectory.

The LfODBN imitated the ZigZag expert similar to the LSTM. However, it performed even worse by choosing to stand still instead of moving downwards/upwards occasionally. Moreover, it sometimes alternated between moving right and left before moving one step down or up. This makes it perform objectively worse than the LSTM and TB agents.

Not a single performance metric in Table 5.21 agrees completely with everything we observed. This implies that neither our proposed $\chi^{2}$ metrics nor the metrics currently used to measure the performance of imitation learning agents are sufficient to evaluate the imitation performance of agents at imitating all experts. Hence, this indicates that sometimes we may need to design performance metrics associated with the expert being imitated rather than using generic metrics when evaluating the imitation performance of agents.

Fixed Sequence Expert As expected, the MLP agent failed to imitate the Fixed Sequence expert. It moved up for 16 steps, then right for 8 steps, and lastly, up for the remainder of its trajectory. This is nowhere near the pattern of the Fixed Sequence 


\begin{tabular}{|c|c|c|c|c|c|c|c|c|}
\hline \multirow{3}{*}{$\begin{array}{l}\text { Agent } \\
\text { MLP }\end{array}$} & \multirow{3}{*}{$\begin{array}{l}\text { Macro } \\
\text { F1 } \\
0.1\end{array}$} & \multirow{2}{*}{\multicolumn{2}{|c|}{$\begin{array}{l}\text { Monte } \\
\text { dCarlo } \\
\text { Dis- } \\
\text { tance } \\
\end{array}$}} & \multirow{3}{*}{$\begin{array}{l}\chi_{P(S)}^{2} \\
1942\end{array}$} & \multirow{3}{*}{$\begin{array}{l}\chi_{P(A)}^{2} \\
1048\end{array}$} & \multirow{2}{*}{\multicolumn{3}{|c|}{$\chi_{P\left(S_{t}, S_{t-1}\right)}^{2} \chi_{P\left(A_{t}, A_{t-1}\right.}^{2} \chi_{P(A, S)}^{2}$}} \\
\hline & & & & & & & & \\
\hline & & 0.1 & 6.84 & & & 1944 & 1631 & 1943 \\
\hline LSTM & 0.28 & 0.28 & 4.62 & 774 & 14.2 & 1136 & 476 & 1093 \\
\hline TB & 0.1 & 0.1 & 6.92 & 667 & 1333 & 1075 & 1998 & 2000 \\
\hline $\begin{array}{l}\text { N- } \\
\text { Ordered } \\
(n= \\
11)\end{array}$ & 0.6 & 0.6 & 2.21 & 45.4 & 20.2 & 144 & 452 & 199 \\
\hline LfODBN & 0.18 & 0.23 & 6.19 & 926 & 106 & 1067 & 918 & 1519 \\
\hline
\end{tabular}

Table 5.22: The results of imitating the Fixed Sequence expert.

agent that is 21 actions long.

The LSTM agent somewhat imitated the fixed sequence of actions of the expert. However, it had frequent hiccups when imitating the Fixed Sequence expert, where it performed an incorrect action then returned to perform a correct sub-sequence of the main sequence of actions the Fixed Sequence expert performs.

The TB agent also failed to imitate the Fixed Sequence agent. It moved in the left direction from the start of the trajectory to the end of the trajectory while there was an obstacle in its left direction. Hence, its environment state did not change from the start to the end of its trajectory. This is even worse than the MLP agent's performance.

The n-ordered agent was the best technique at imitating the Fixed Sequence agent. It performed the exact sequence of actions as the expert; however, it was off by one action at the start of its trajectory. It moved in the left direction at the start of its run instead of going up; however, on its second action, it moved up and continued 
performing the correct sequence of actions till the end of its trajectory.

The LfODBN agent performed very similarly to the LSTM agent. It somewhat imitated the sequence of actions but with many more mistakes compared to the LSTM agent. In addition, it often selected to stand still, which is an action that does not exist in our sequence of actions.

The best performance metric to use in order to evaluate the performance of each

agent is clearly the $\chi_{P\left(A_{t}, A_{t-1}\right)}^{2}$ in Table 5.22. Its results are also inline with what we observed. The best performing agent is the n-ordered agent, followed by the LSTM agent, then the LfODBN agent. The worst agents at imitating this expert were the MLP and the TB agents, which were nowhere near the behavior of the Fixed Sequence expert.

\subsubsection{Conclusion}

In this experiment, we saw how none of the imitation learning techniques we used was universally better at imitating all experts. However, we had promising results from the qualitative results of the LSTM agent at imitating the ZigZag expert. On the other hand, the performance metrics we used were not conclusive on which agent performed the best in the case of the ZigZag expert. This experiment showed us how difficult it is to find a performance metric that is generic and applicable to all experts. It also showed how our proposed performance metrics may be insufficient at evaluating the performance of agents at imitating certain experts such as the ZigZag expert. Thus, we may need to design performance metrics per expert we imitate to properly evaluate each technique.

Both our quantitative and qualitative results of the ZigZag expert showed that the LSTM and TB were the best techniques at imitating the expert. This was in line 
with what we hypothesized. However, we saw that LSTM was able to perform better than TB and LfODBN at imitating the Fixed Sequence expert despite not including the previous action in its computation of its hidden internal state; which is not what we expected. This is likely due to the recency bias assumption that TB makes and LSTM does not. On the other hand, the n-ordered agent was able to imitate the Fixed Sequence expert almost perfectly, since it weighs all previous $n$ actions equally when selecting the next action to take.

\subsection{Conclusion}

In this chapter, we learnt about the effect of DAgger on imitation learning techniques, the inadequacy of offline testing methodologies, and the performance of our LSTM network in the continuous RoboCup soccer domain and the discrete vacuum cleaner domain.

We saw how DAgger negatively affected the performance of imitation learning techniques that consider past actions, such as the n-ordered similarity CBR technique in the RoboCup soccer domain. We also showed how DAgger improved the performance of imitation learning techniques that did not consider past actions when selecting which action to perform in the RoboCup soccer domain. Moreover, we showed how LSTM was able to outperform all the state-of-the-art imitation learning techniques we used at imitating the experts of the continuous RoboCup soccer domain.

In the vacuum cleaner domain, we showed how our online testing methodology provided us with consistent and reliable results. We also showed how the offline testing methodology adopted in the work of Gunaratne et al. [4] and the work of other authors such as Ontañón et al. [1] is not reliable to obtain conclusive results that match the 
eye-test of the agents. Moreover, we saw how LfODBN, TB and n-ordered similarity CBR were better at imitating certain experts in the discrete vacuum cleaner domain in comparison to LSTM networks. This is due to the stochastic component of some of those experts, and the reliance of the Fixed Sequence expert on the previous actions instead of the previous environment states when deciding which action to take.

In addition to what we learnt in the vacuum cleaner domain, we saw how no performance metric was self-sufficient to conclude which expert performed better at imitating the ZigZag expert. Therefore, we may need to design performance metrics that are specific to the expert we are imitating. However, we believe that our proposed metrics were a decent attempt at proposing generic performance metrics that are sufficient to assess most experts. This was evident in most of the experts we used in our experiments, with the only exception being the ZigZag expert. 


\section{Chapter 6}

\section{Conclusion and Future Work}

\subsection{Conclusion}

The two main objectives of this thesis were (1) to propose performance metrics and a testing methodology that provide results that agree with the qualitative performance of imitation learning agents at imitating a particular expert, and (2) to compare the performance of LSTM networks to state-of-the-art imitation learning techniques. We made use of both reactive and state-based computer experts with well defined behavior in the RoboCup soccer and vacuum cleaner domains. Consequently, this provided us with a set of expected behaviors that we can compare our imitation learning agents' behavior to. Hence, we were able to qualitatively assess the performance of each imitation learning agent at imitating each expert.

We proposed five metrics that compare different distributions within the trajectory of an agent to the trajectory of the expert, and proposed running the trained agents in the environment in order to obtain our results in Chapter 4. Since the imitation learning agents were evaluated on their own trajectory instead of the trajectory of the 
experts, our experiment in Section 5.5.2 showed how this testing methodology produced results that are consistent with how well the agents imitated the expert's behavior. In contrast, the traditional offline testing methodology evaluates the performance of the agents on a testing set of the expert's trajectory. However, when the agent is deployed, its trajectory is dependent on its actions. Hence, the offline testing methodology fails to produce results that are consistent with the qualitative performance of the agents.

Moreover, our proposed performance metrics, that are meant to be used with our online testing methodology, measure the distance between the environment state distribution $P(S)$, the action distribution $P(A)$, the joint environment state and previous environment state distribution $P\left(S_{t}, S_{t-1}\right)$, the joint action and previous action distribution $P\left(A_{t}, A_{t-1}\right)$, and the joint action and environment state distribution $P(A, S)$ of the trained agent's trajectory and the expert's trajectory. Using prior knowledge about the behavior of an expert, we were often able to select a distribution that ranked the imitation learning agents according to their qualitative performance at imitating the expert in the environment. For instance, the Smart Random expert in Section 5.5.2 selects an action randomly most of the time; hence, our performance metric $\chi_{P(A)}^{2}$ was consistent with the qualitative performance of each technique at imitating the Smart Random expert. However, we still found a case in our experiment in Section 5.5.3 where none of our metrics were self-sufficient to dictate which technique performed better. Specifically, in the case of the ZigZag expert, it was difficult to qualitatively discern which technique performed better than the other when comparing LfODBN, TB and LSTM. Hence, our performance metrics were also unable to concretely conclude which of those techniques performed better at imitating the ZigZag expert. This indicates the occasional need to design performance metrics around the expert rather than the use of generic metrics such as the performance 
metrics we proposed. However, when the expert's behavior is unknown to us, using $\chi_{P(A)}^{2}$ and $\chi_{P(S)}^{2}$ can provide us with information on how well an imitation learning agent generalized the behavior of the expert. When an important part of the expert's unknown behavior consists of sequential actions or environment states, $\chi_{P\left(A_{t}, A_{t-1}\right)}^{2}$ and $\chi_{P\left(S_{t}, S_{t-1}\right)}^{2}$ provides us with information on how well the imitation learning generalized the sequential component of the expert's behavior.

We also compared our LSTM network to other state-of-the-art techniques in both the RoboCup soccer domain and the Vacuum Cleaner domain. This required us to create a new model of the RoboCup soccer environment state vector that preserves the continuous nature of the domain and introduce new RoboCup soccer state-based experts. We showed how the LSTM network outperformed all other state-of-the-art imitation learning techniques that we applied in the RoboCup soccer domain when imitating the state-based experts we designed. On the contrary, we showed how our LSTM network may under-perform at imitating certain state-based experts compared to state-of-the-art techniques when the domain is discrete. All the experts where the LSTM network under-performed relative to other techniques had stochastic behavior or behavior that was heavily reliant on past actions. Thus, we recommend using a LSTM network when imitating state-based experts that are deterministic and reliant on past environment states rather than past actions. Moreover, we recommend using a LSTM network when the environment state space is continuous. On the other hand, we recommend using LfODBN when imitating stochastic experts in a discrete domain. Hence, the best imitation learning technique to use depends on the behavior of the expert imitated and the properties of its environment.

In addition to the main objectives of this thesis, we performed an experiment to see how DAgger affects imitation learning techniques in the RoboCup soccer domain. 
We showed how DAgger improves the performance of TB because of its recency bias. We also showed how DAgger negatively affects the performance of state-based CBR similarity metrics, such as the n-ordered similarity. This is because those metrics consider past actions with equal importance to past environment states. This results in the training trajectories added by DAgger to be more distant from the trajectory of the agent (which is performing an incorrect action); hence, the agent does not correct its action.

\subsection{Future Work}

Our proposed performance metrics use the $\chi^{2}$ statistic to compute the distance between two distributions: a distribution of the agent and a distribution of the expert. The $\chi^{2}$ statistic has no upper-bound and therefore, is difficult to normalize. We can use

a library to normalize our metrics using the $\chi^{2}$ value and the respective degrees of freedom; however, there is no equation to directly normalize our metrics. Hence, it is worth investigating which statistic is better suited for measuring the distance between two distributions as a performance metric. Comparing the Kullback-Leibler divergence [36] and the Kolmogorov-Smirnov test [37] to $\chi^{2}$ as a performance metric is an area of future work.

Moreover, LSTM networks are one example of RNN networks. There are other examples of RNN networks such as Gated Recurrent Unit (GRU) [38]. It would be worth comparing different RNNs to see which RNN model works best in the field of state-based imitation learning.

Another area where future work can be done is improving the LSTM model at imitating state-based experts by including its selected action at time $t-1$ in the 
computation of its hidden internal state. It would be interesting to compare this LSTM model to the traditional LSTM model that we used in the experiments of this thesis and to see whether it improves its imitation learning performance or not. Additionally, testing how DAgger affects this model's performance is another area where future work can be done. This experiment could further affirm our theory behind why state-based CBR is negatively affected by DAgger.

In addition to what we mentioned, using transformers that are currently being used in NLP [39] to learn state-based behavior is another area that is worth investigating. Their ability to generalize is very promising and they may perform better than LSTM networks at imitating the state-based experts of the RoboCup soccer domain. 


\section{Bibliography}

[1] Santiago Ontañón, José L. Montaña, and Avelino J. Gonzalez. A dynamic-bayesian network framework for modeling and evaluating learning from observation. Expert Syst. Appl., 41(11):5212-5226, 2014.

[2] Amrik Sacha Elapata Gunaratne, Babak Esfandiari, and Ali Fawaz. A case-based reasoning approach to learning state-based behavior. In Proceedings of the 31st International Florida Artificial Intelligence Research Society Conference, FLAIRS 2018, pages 377-382, jan 2018.

[3] Cristina Tîrnăucă, José L Montaña, Santiago Ontañón, Avelino J González, and Luis M Pardo. Behavioral modeling based on probabilistic finite automata: An empirical study. Sensors, 16(7):958, 2016.

[4] Amrik Sacha Elapata Gunaratne, Babak Esfandiari, and Caleb Chan. Towards a framework for testing learning from observation of state-based agents. In $A A A I$ Spring Symposium - Technical Report, pages 499-505, jan 2017.

[5] Brenna D Argall, Sonia Chernova, Manuela Veloso, and Brett Browning. A survey of robot learning from demonstration. Robotics and autonomous systems, 57(5):469-483, 2009. 
[6] Michael W Floyd, Babak Esfandiari, and Kevin Lam. A case-based reasoning approach to imitating robocup players. In FLAIRS Conference, pages 251-256, 2008.

[7] P.K. Pook and D.H. Ballard. Recognizing teleoperated manipulations. In [1993] Proceedings IEEE International Conference on Robotics and Automation, pages 578-585 vol.2, 1993.

[8] Peter Pastor, Heiko Hoffmann, Tamim Asfour, and Stefan Schaal. Learning and generalization of motor skills by learning from demonstration. In 2009 IEEE International Conference on Robotics and Automation, pages 763-768. IEEE, 2009 .

[9] T INAMURA. Acquisition of probabilistic behavior decision model based on the interactive teaching method. In Proc. 9th Int. Conf. on Advanced Robotics, pages 523-528, 1999.

[10] Robert Bogue. Domestic robots: Has their time finally come? Industrial Robot: An International Journal, 2017.

[11] Dario Amodei, Chris Olah, Jacob Steinhardt, Paul Christiano, John Schulman, and Dan Mané. Concrete problems in ai safety. arXiv preprint arXiv:1606.06565, 2016.

[12] Brenna D Argall, Sonia Chernova, Manuela Veloso, and Brett Browning. A survey of robot learning from demonstration. Robotics and autonomous systems, 57(5):469-483, 2009.

[13] Michael Wooldridge. Introduction to Multiagent Systems. John Wiley \& Sons, Inc., USA, 2001. 
[14] Sepp Hochreiter and Jürgen Schmidhuber. Long short-term memory. Neural Computation, 9(8):1735-1780, 1997.

[15] Michael I Jordan. Serial order: A parallel distributed processing approach. In Advances in psychology, volume 121, pages 471-495. Elsevier, 1997.

[16] Aytug Onan and Mansur Alp Toçoğlu. A term weighted neural language model and stacked bidirectional lstm based framework for sarcasm identification. IEEE Access, 9:7701-7722, 2021.

[17] Alex Graves. Generating sequences with recurrent neural networks. arXiv preprint arXiv:1308.0850, 2013.

[18] Shweta Yadav, Asif Ekbal, Sriparna Saha, Ankit Kumar, and Pushpak Bhattacharyya. Feature assisted stacked attentive shortest dependency path based bi-lstm model for protein-protein interaction. Knowledge-Based Systems, 166:18$29,2019$.

[19] Yu-Lun Hsieh, Yung-Chun Chang, Nai-Wen Chang, and Wen-Lian Hsu. Identifying protein-protein interactions in biomedical literature using recurrent neural networks with long short-term memory. In Proceedings of the eighth international joint conference on natural language processing (volume 2: short papers), pages 240-245, 2017.

[20] Michael W Floyd and Babak Esfandiari. Learning state-based behaviour using temporally related cases. In Proceedings of the Sixteenth UK Workshop on Case-Based Reasoning, pages 34-45, 2011.

[21] Stephane Ross, Geoffrey Gordon, and Drew Bagnell. A reduction of imitation learning and structured prediction to no-regret online learning. In Proceedings of 
the Fourteenth International Conference on Artificial Intelligence and Statistics, volume 15 of Proceedings of Machine Learning Research, pages 627-635, Fort Lauderdale, FL, USA, 11-13 Apr 2011. PMLR.

[22] Pieter Abbeel, Adam Coates, and Andrew Y Ng. Autonomous helicopter aerobatics through apprenticeship learning. The International Journal of Robotics Research, 29(13):1608-1639, 2010.

[23] Alex Sherstinsky. Fundamentals of recurrent neural network (rnn) and long shortterm memory (lstm) network. Physica D: Nonlinear Phenomena, 404:132306, 2020.

[24] Jeffrey L Elman. Finding structure in time. Cognitive science, 14(2):179-211, 1990.

[25] Felix A Gers, Jürgen Schmidhuber, and Fred Cummins. Continual prediction using lstm with forget gates. In Neural Nets WIRN Vietri-99, pages 133-138. Springer, 1999.

[26] irobot@: Robot vacuum and mop. https://www.irobot.com/. Accessed: July 2021.

[27] Robocup federation official website. https://www.robocup.org/. Accessed: June 2021.

[28] Nir Friedman, Dan Geiger, and Moises Goldszmidt. Bayesian network classifiers. Machine learning, 29(2):131-163, 1997. 
[29] Zoubin Ghahramani. Learning dynamic bayesian networks. In International School on Neural Networks, Initiated by IIASS and EMFCSC, pages 168-197. Springer, 1997.

[30] Yoshua Bengio and Paolo Frasconi. Input-output hmms for sequence processing. IEEE Transactions on Neural Networks, 7(5):1231-1249, 1996.

[31] Arthur P Dempster, Nan M Laird, and Donald B Rubin. Maximum likelihood from incomplete data via the em algorithm. Journal of the Royal Statistical Society: Series B (Methodological), 39(1):1-22, 1977.

[32] John Shafer, Rakesh Agrawal, Manish Mehta, et al. Sprint: A scalable parallel classifier for data mining. In Vldb, volume 96, pages 544-555. Citeseer, 1996.

[33] Michael R Berthold and Jay Diamond. Constructive training of probabilistic neural networks. Neurocomputing, 19(1-3):167-183, 1998.

[34] Martin Riedmiller and Heinrich Braun. A direct adaptive method for faster backpropagation learning: The rprop algorithm. In IEEE international conference on neural networks, pages 586-591. IEEE, 1993.

[35] Krzysztof Langner. Krislet (java). https://www.ida.liu.se/ frehe08/ RoboCup/Libs/libsv5xx.html, 1999. Accessed: July 2021.

[36] Solomon Kullback and Richard A Leibler. On information and sufficiency. The annals of mathematical statistics, 22(1):79-86, 1951.

[37] Frank J Massey Jr. The kolmogorov-smirnov test for goodness of fit. Journal of the American statistical Association, 46(253):68-78, 1951. 
[38] Junyoung Chung, Caglar Gulcehre, KyungHyun Cho, and Yoshua Bengio. Empirical evaluation of gated recurrent neural networks on sequence modeling. arXiv preprint arXiv:1412.3555, 2014.

[39] Ashish Vaswani, Noam Shazeer, Niki Parmar, Jakob Uszkoreit, Llion Jones, Aidan N Gomez, Łukasz Kaiser, and Illia Polosukhin. Attention is all you need. In Advances in neural information processing systems, pages 5998-6008, 2017. 